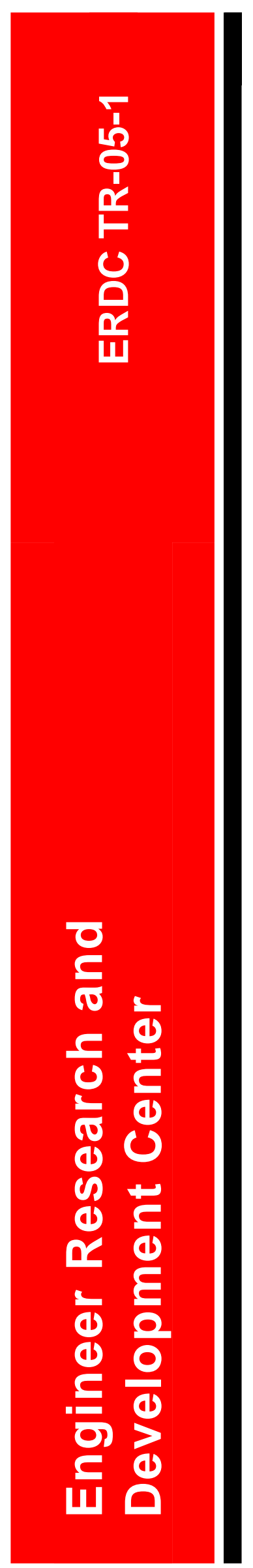

\title{
Using High Spatial Resolution Digital Imagery
}

Michael V. Campbell, Robert L. Fischer, Timothy

February 2005

Pangburn and Mark J. Hardenberg 


\begin{abstract}
This document is the culmination of over 3 years worth of applied research. The overall objective of this multi-year investigation was to assess the utility of high spatial resolution digital imagery to Corps civil works operations. The sources of remotely sensed data used in this research effort include digital airborne multispectral imaging technology, digital airborne hyperspectral imaging technology, and digital satellite multispectral images. System selection was based on the anticipated benefits derived from the acquisition and processing of the unique high spatial resolution images, from each remotely sensed data source, at each independent study site. The project developed and implemented four applications to evaluate the use of high resolution digital remotely sensed data in standard Corps operations. This document summarizes the four projects. The intended audience for this document includes natural resource managers, biologists, ecologists, GIS specialists, and others that are already using or are potentially planning to use high spatial resolution digital remotely sensed imagery for current or future projects. The image and map products, including both raster and vector formatted data, are not provided with this report. The authors can provide the reader access to all of the imagery and final maps.
\end{abstract}

DISCLAIMER: The contents of this report are not to be used for advertising, publication, or promotional purposes. Citation of trade names does not constitute an official endorsement or approval of the use of such commercial products. All product names and trademarks cited are the property of their respective owners. The findings of this report are not to be construed as an official Department of the Army position unless so designated by other authorized documents. 
ERDC TR-05-1

February 2005

\section{Using High Spatial Resolution Digital Imagery}

\section{Michael V. Campbell, ${ }^{*}$ Robert L. Fischer, ${ }^{*}$ Timothy Pangburn, ${ }^{t}$} and Mark J. Hardenberg**

*Topographic Engineering Center

7701 Telegraph Road

Bldg. 2592

Alexandria, Virginia 22315

${ }^{\dagger}$ Cold Regions Research and Engineering Laboratory

72 Lyme Road

Hanover, NH 03755

**Information Technology Laboratory

72 Lyme Road

Hanover, NH 03755

Approved for public release; distribution is unlimited.

Prepared for U.S. Army Corps of Engineers

Washington, DC 20314-1000 



\section{CONTENTS}

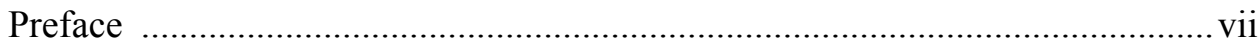

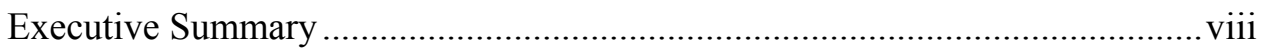

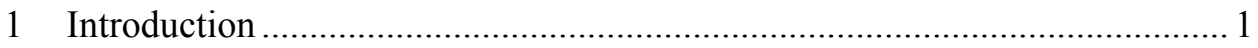

1.1 Land Cover Classification of Reference Wetlands ................................... 2

1.2 Enhanced Levee Inspections.............................................................. 3

1.3 Invasive Species Mapping at Lake Okeechobee, Florida ......................... 3

1.4 Detailed Wetland Vegetation Mapping Over Blackwater

Wildlife Refuge.............................................................................. 4

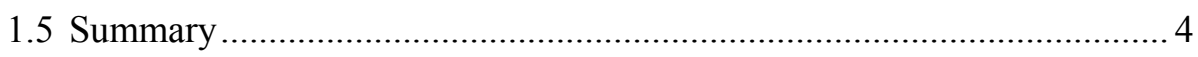

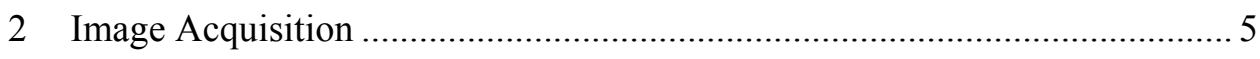

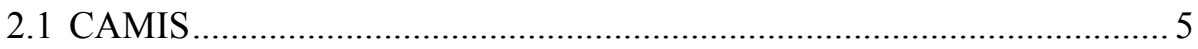

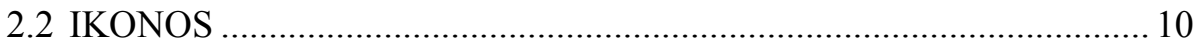

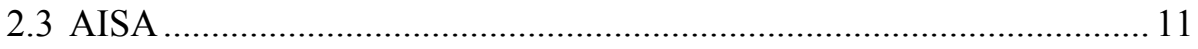

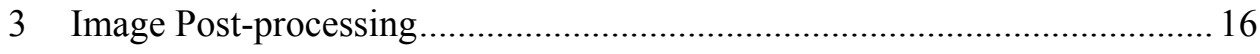

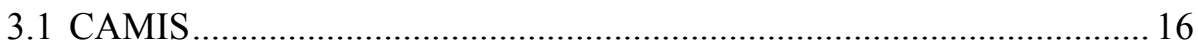

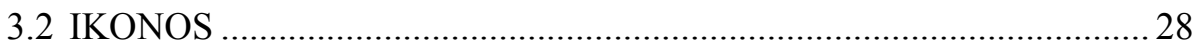

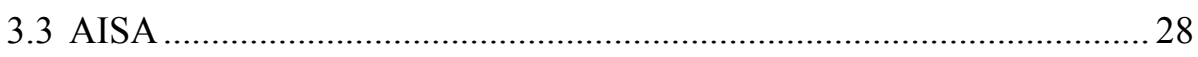

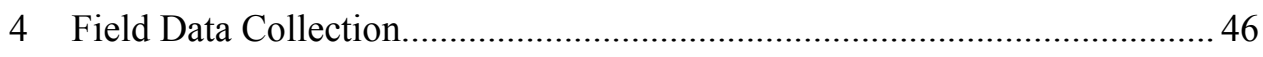

4.1 Invasive Species Mapping at Lake Okeechobee, Florida ...................... 46

4.2 Wetland Vegetation Mapping Over Blackwater Wildlife Refuge .......... 46

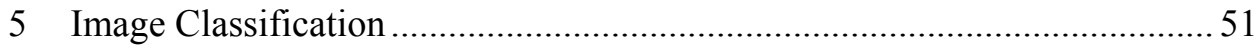

5.1 Poplar Island Unsupervised Classification .......................................... 51

5.2 Invasive Species Mapping at Lake Okeechobee, Florida ....................... 59

5.3 Wetland Vegetation Mapping Over Blackwater Wildlife Refuge .......... 64

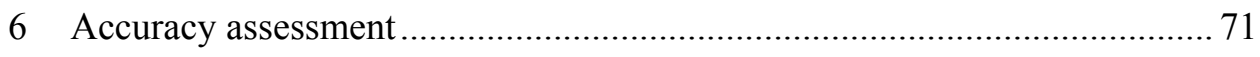

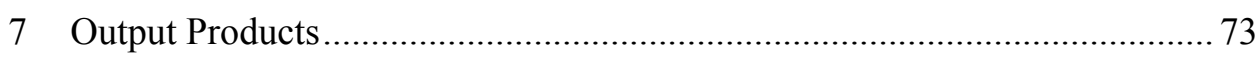

7.1 Image Quality for Poplar Island Restoration ...................................... 73

7.2 Land Cover Classes for Poplar Island Restoration ............................... 75

7.5 Utility For Arcview Application for Enhanced Levee Inspections......... 79 
7.7 Results for Wetland Vegetation Mapping Over Blackwater Wildlife Refuge 81

8 Costs 84

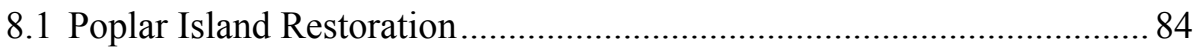

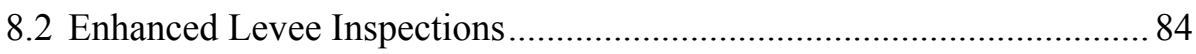

8.3 Invasive Species Mapping at Lake Okeechobee, Florida ...................... 86

8.4 Wetland Vegetation Mapping Over Blackwater Wildlife Refuge .......... 87

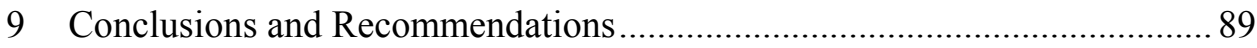

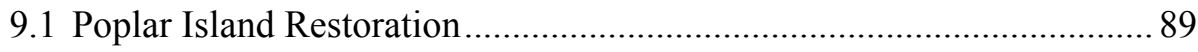

9.2 Enhanced Levee Inspections ................................................................. 90

9.3 Invasive Species Mapping at Lake Okeechobee, Florida ...................... 90

9.4 Wetland Vegetation Mapping Over Blackwater Wildlife Refuge .......... 91

\section{ILLUSTRATIONS}

Figure 1. CAMIS model 4768P airborne digital multispectral sensor................... 6

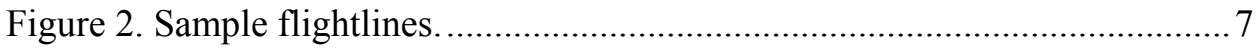

Figure 3. Orientation of the nine flightlines required to acquire a complete

CAMIS image set over the Sny levee.................................................... 8

Figure 4. True-color and false color composites of Fisheating Bay, Monkey Box,

and Moonshine Bay acquired 9 December 2001....................................... 9

Figure 5. IKONOS image of western Lake Okeechobee, acquired July 2002.....10

Figure 6. Direct-vision, dispersing prism-grating-prism spectrograph used in the

AISA sensor.

Figure 7. Location and orientation of AISA flightlines over Blackwater

Wildlife Refuge.

Figure 8. Raw false color CAMIS frame and band registered false color CAMIS

frame Knapps Narrows, frame 106.

Figure 9. Band registered true color CAMIS frame and radiometrically corrected true color CAMIS frame, Hell Hook Marsh, flightline 2, frame 108............ 18

Figure 10. True color, three-frame mosaic of Piney Creek (PIN) wetland.......... 19

Figure 11. Knapps Narrows mosaic before and after geometric registration to DOQQ.

Figure 12. Piney Creek mosaic before and after geometric registration to digital

$7.5^{\prime}$ topographic quadrangle.

Figure 13. Knapps Narrows and PIN mosaics after subsetting......................... 21 
Figure 14. Examples of poor, and good geometric registration.........................23

Figure 15. 4 September 2001 geo-registered mosaics. ................................... 24

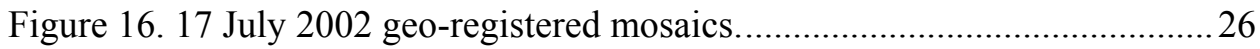

Figure 17. Examples of the geometric quality of the AISA imagery...................29

Figure 18. True color mosaic of uncorrected lines BLINE7E and BLINE8W ....3 31

Figure 19. False color mosaic of uncorrected lines BLINE7E and BLINE8W ... 32

Figure 20. Flightlines BLINE7E and BLINE8W and their associated mean reflectance spectra, including background pixels.

Figure 21. Flightlines BLINE7E and BLINE8W and their associated mean reflectance spectra, without background pixels

Figure 22. Example of NDVI image. Graphs show floating point NDVI values and NDVI values scaling to 8-bit data.

Figure 23. Flightline BLINE7E and BLINE8W and their associated mean reflectance spectra after removal of water pixels and reduction in data dimensionality.

Figure 24. Histogram equalization results between BLINE7E and BLINE8W... 42

Figure 25. Southern mosaic - flightlines BLINE7E through BLINE20E .......... 43

Figure 26. Final mosaics for flightlines 1 through 5 acquired on 30 June 2000.. 44

Figure 27. Location of field plots established during 9 December 2001 CAMIS

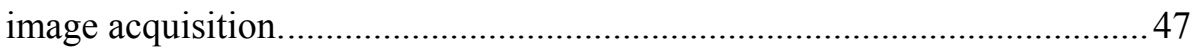

Figure 28. Location of training sites within western part of northern mosaic..... 48

Figure 29. Location of training sites adjacent to Shorters Warf Road................ 49

Figure 30. ArcView shapefile polygons used to collect training spectra for

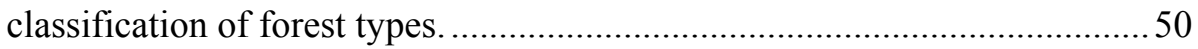

Figure 31. NDVI image, NDVI histogram and primary NVDI masks...............53

Figure 32. Primary image segments after NDVI masking................................54

Figure 33. Results of unsupervised classification of each primary segment. ......55

Figure 34. Class map of Knapps Narrows - full resolution................................56

Figure 35. Class map of Knapps Narrows after application of minimum mapping

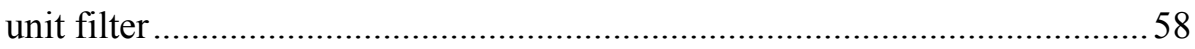

Figure 36. Supervised classification results of CAMIS mosaics after application of the 144 pixel minimum mapping unit filter. ...........................................62 62

Figure 37. Unsupervised classification results of IKONOS image after application of the nine-pixel minimum mapping unit filter.

Figure 38. Southern Mosaic - flightlines BLINE7E through BLINE20E. falsecolor composite, and true-false-color composite.........................................66

Figure 39. Spectral plots of low reflectance signatures used to classify the southern mosaic.

Figure 40. Spectral plots of high reflectance signatures used to classify the southern mosaic. 
Figure 41. Mosaic of Hell Hook Marsh showing significant holidays between flightlines.

Figure 42. False color and true color mosaics for Spillway 6 site, within Poplar Island restoration/construction area........................................................ 75

Figure 43. False color composite for Cabin Cove. ............................................ 77

Figure 44. Class map for Cabin Cove after application of minimum mapping unit

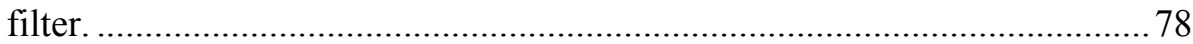

Figure 45. Final three class thematic product depicting the distribution of the invasive species of interest to the District ................................................... 80

Figure 46. Final vegetation class map for southern mosaic................................ 82

Figure 47. Final vegetation class map for northern mosaic ............................. 83 


\section{PREFACE}

This report was prepared by Michael V. Campbell, Topographic, Imagery and Geospatial Research Division, and Robert L. Fischer, Topographic, Imagery and Geospatial Research Division, Topographic Engineering Center, Timothy Pangburn, Chief, Remote Sensing/Geographic Information System Center, Cold Regions Research and Engineering Laboratory, and Mark J. Hardenberg, Technical Publications Editor, Information Technology Laboratory, U.S. Army Engineer Research and Development Center.

The project was funded by the USACE Headquarters Geospatial Technology Research and Development Program.

This report was prepared under the general supervision of Dr. Lance Hansen, Deputy Director, and James Wuebben, Acting Director, CRREL.

The Commander and Executive Director of the Engineer Research and Development Center is COL James R. Rowan, EN. The Director is Dr. James R. Houston. 


\section{EXECUTIVE SUMMARY}

This document is presented as the culmination of over 3 years worth of applied research. The project was funded by the USACE Headquarters Geospatial Technology Research and Development Program. The overall objective of this multi-year investigation was to assess the utility of high spatial resolution digital imagery to Corps civil works operations. The sources of remotely sensed data used in this research effort include:

- Digital airborne multispectral imaging technology.

- Digital airborne hyperspectral imaging technology.

- Digital satellite multispectral images.

System selection was based on the anticipated benefits derived from the acquisition and processing of the unique high spatial resolution images, from each remotely sensed data source, at each independent study site.

The project developed and implemented four applications to evaluate the use of high resolution digital remotely sensed data in standard Corps operations. This document summarizes the four projects.

\section{Detailed Wetlands Mapping using Airborne Multispectral Imagery (Balti- more District)}

The goal of the Poplar Island Restoration Project is to rebuild a naturally eroded group of islands within the upper Chesapeake Bay. The District engineers, hydrologists, and biologist are tasked with constructing a variety of functioning estuarine wetland systems. Existing wetland sites, distributed near the construction site on the Eastern Shore of Maryland, were selected for characterization and monitoring. These functioning sites were designated as reference wetlands and would guide the construction activities on Poplar Island. High spatial resolution digital airborne multispectral imagery was acquired over eight reference wetlands in February of 2001. The images were post-processed into geometrically and radiometrically corrected mosaics. An unsupervised classification approach was employed to delineate 16 land cover classes. These 16 classes provided adequate information to map three primary classes, including persistent water channels and guts, tidal zones (e.g., mudflats) and areas supporting wetland/upland vegetation. The output products included vector formatted ArcView shapefiles for each site. 


\section{Enhance Levee Inspections (Rock Island District)}

The Corps inspects thousands of miles of flood control structures each year. The Rock Island District has implemented an ArcView-based application, complete with real-time GPS navigation, to improve levee inspections. Airborne multispectral imagery of 1-m spatial resolution was acquired over the Sny levee. An evaluation of the complete mosaics determined that even higher resolution data (i.e., smaller pixels) would be necessary to provide the detail needed to delineate anomalies on the structure.

\section{Invasive Species Mapping (Jacksonville District)}

The Aquatic Plant Control Operations Support Center is responsible for managing exotic invasive species within a large wetlands complex distributed throughout the western portion of Lake Okeechobee, Florida. The objective of this research effort was to delineate areas supporting water hyacinth and water lettuce using high resolution multispectral imagery from both an airborne system and from a commercial satellite sensor. The airborne image products suggested that the spectral signatures of many of the marsh plant communities were very similar. The satellite imagery provided a much more cost-effective solution for creating a class map depicting the distribution of invasive species.

Wetlands Mapping with Airborne Hyperspectral Imagery (Baltimore District)

The Blackwater Wildlife Refuge, located on the Eastern Shore of Maryland (Cambridge, Maryland), supports a variety of wetland restoration initiatives. In conjunction with the Fish and Wildlife Service, high resolution airborne digital hyperspectral imagery was acquired over an area covering approximately 70,000 acres. A detailed vegetation map was produced using a limited amount of ground truth samples. The accuracy of the final vegetation classification was relatively low. Many of the classification errors were attributed to the poor radiometric properties of the hyperspectral image mosaic.

The intended audience for this document includes natural resource managers, biologists, ecologists, GIS specialists, and others that are already using or are potentially planning to use high spatial resolution digital remotely sensed imagery for current or future projects. The primary points of contact for questions and comments concerning this document are Michael V. Campbell (703-4286538) and Robert L. Fischer (703-428-7140) of the Topographic, Imagery and Geospatial Research Division, U.S. Army Engineer Research and Development Center, Topographic Engineering Center, located in Alexandria, Virginia. 
The image and map products, including both raster and vector formatted data, are not provided with this report. The authors can provide the reader access to all of the imagery and final maps. Please note that some type of image processing software, such as ERDAS Imagine or RSI ENVI, is best used to display and manipulate the multispectral and hyperspectral images, as well as the final map products. However, standard GIS software, such as ArcGIS, can also display the remotely sensed data. 


\title{
USING HIGH SPATIAL RESOLUTION DIGITAL IMAGERY
}

\author{
MICHAEL V. CAMPBELL, ROBERT L. FISCHER, TIMOTHY PANGBURN, \\ AND MARK J. HARDENBERG
}

\section{INTRODUCTION}

The newest digital airborne remote sensing systems combine the multispectral capabilities of commercial satellite sensors with the temporal and spatial flexibility of traditional aerial photographic systems. The high spatial resolution imagery provided by these systems greatly enhances environmental monitoring strategies. Sub-meter pixel resolutions increase the detail of surface features. However, too much data can degrade the accuracy and the utility of the final map product. Therefore, the effective use of high-resolution digital airborne imagery requires improved processing techniques that ensure accurate representation of the land cover classes of interest. Post-processing algorithms to remove radiometric and geometric distortions, which are typically present in all airborne images, are essential and must be developed.

The Topographic Engineering Center (TEC), part of the U.S. Army Engineer Research and Development Center (ERDC), has been developing digital multispectral airborne remote sensing capabilities since 1994. The first system used was the Digital Multispectral Video System (DMSV). Developed by SpecTerra Systems (Canberra, Australia), DMSV imagery was shown to be successful in a variety of environmental applications, such as detecting oil spills, characterizing acid mine drainage, and evaluating wetlands.

In 1998, TEC purchased the Computerized Airborne Multicamera Imaging System (CAMIS) from Flight Landata, Inc. (Lawrence, Massachusetts). This system used an enhanced lens design and an improved user interface, resulting in more efficient data acquisition and improved image quality. The CAMIS system has acquired imagery for invasive plant species mapping, enhanced vegetation feature extraction, and ecosystem/ecotone characterization. 
TEC research scientists have developed improved post-processing routines addressing radiometric distortions, which are typically present in all airborne images. This document is the culmination of over three years worth of applied research. The overall objective of this multi-year investigation was to assess the utility of high spatial resolution digital imagery to Corps civil works operations. The sources of remotely sensed data used in this research effort include:

- Digital airborne multispectral imaging technology.

- Digital airborne hyperspectral imaging technology.

- Digital satellite multispectral imaging technology.

Systems were selected on the basis of the anticipated benefits derived from the acquisition and processing of the unique high spatial resolution images, from each remotely sensed data source, at four independent study sites - wetland characterization for the Poplar Island Restoration project in the Baltimore District, levee inspection in the Rock Island District, invasive species mapping in the Jacksonville District, and the wetland vegetation mapping in the Blackwater Wildlife Refuge, also in the Baltimore District.

The project developed and implemented four applications to evaluate the use of high resolution digital remotely sensed data in standard Corps operations.

\subsection{Land Cover Classification of Reference Wetlands}

The objective of this pilot project for Poplar Island Restoration was delineating three primary land cover types within selected estuarine wetlands. The three land cover categories were:

1. Persistent water bodies (open water, channels, guts, ponds, and potholes).

2. Intertidal mudflats (both vegetated and non-vegetated).

3. Wetland and upland plant communities.

The detailed and long-term nature of this ongoing wetland restoration program provides an excellent test site for evaluating the utility of high resolution mapping techniques.

The restoration project design includes the construction of various hydrologic features (ponds, dendritic guts, or channels). The relative proportion (i.e., surface area) and configuration of these water bodies within the proposed wetland and upland plant communities are critical design parameters. District staff concluded that the natural conditions present in existing intertidal wetlands near the project site would supply estimates of the hydrologic characteristics, such as land-to-water ratios and channel dendricity, required throughout the construction 
site. Corps personnel initially identified nearby estuarine wetlands as reference sites to aid in the design and installation of proposed constructed wetlands on Poplar Island. A preliminary cooperative effort between the Baltimore District and the National Marine Fisheries Service (NMFS) was underway to characterize the reference wetlands when TEC proposed the incorporation of high-resolution airborne multispectral imagery.

\subsection{Enhanced Levee Inspections}

The Corps of Engineers is responsible for the construction and maintenance of thousands of miles of levees throughout the continental United States. The Rock Island District maintains more than 546 combined miles of levees and concrete flood walls along the Mississippi and Illinois rivers and their tributaries. Periodic inspections of these flood control structures are mandatory. The District has recently developed and implemented an ArcView GIS application to assist with levee inspections.

The objective of this applied research effort was to evaluate the cost effectiveness of utilizing high spatial resolution airborne digital multispectral imagery to enhance levee inspection procedures. Cost effectiveness was evaluated based on total cost to acquire and post-process the airborne images, and the benefit that the mosaics provided to the levee inspection procedures as enhanced background layers in the ArcView application.

\subsection{Invasive Species Mapping at Lake Okeechobee, Florida}

Western Lake Okeechobee supports a complex system of freshwater wetland and aquatic plant communities. Invasive and undesirable plant species are a significant problem throughout Southern Florida, especially within the diverse communities that characterize Lake Okeechobee. Examples of invasive exotics include Hydrilla verticillata, water hyacinth (Eichhornia crassipes), water lettuce (Pistia stratiotes), and Brazilian pepper (Schinus terebinthifolius). Invasive species management practices require the initial step of delineating the areal extents of plant populations.

The objective of this applied research effort was to employ high spatial resolution digital airborne and satellite-borne multispectral imagery to identify and delineate various exotic and native plant communities within selected study sites over western Lake Okeechobee. 


\subsection{Detailed Wetland Vegetation Mapping Over Blackwater Wildlife Refuge}

The Corps of Engineers Baltimore District has recently started the Blackwater Refuge, Dorchester County, Maryland, Section 206 Small Ecosystem Restoration project.

Accurate and detailed land use and land cover base maps are critical to monitoring and evaluating both the short-term and long-term environmental impacts of the restoration project.

The objective of this applied research effort is to employ industry standard techniques to create a detailed vegetation classification of the entire refuge. Innovative hyperspectral image processing algorithms and techniques may be developed and implemented, as necessary.

\subsection{Summary}

The following will attempt to synthesize the experiences obtained at the four above study sites to provide general guidance about how to obtain images, postprocess them, collect field data, classify the images, assess their accuracy and output products, and provide some guidelines as to cost.

ERDC/CRREL Letter Report LR-04-25 contains separate, expanded sections about each of the study sites that include more and detailed information. 


\section{IMAGE ACQUISITION}

\subsection{CAMIS}

A flight mission is needed to acquire high-resolution imagery using the CAMIS model 4768P, developed by Flight Landata (Lawrence, Massachusetts) (Fig. 1). Unlike other currently available airborne digital camera systems, which employ a single CCD technology, the CAMIS optical head consists of four Sony $\mathrm{XC} 8500$ progressive scan cameras mounted on a sited solid base. Each camera is sensitive in the range of 350-1100 $\mathrm{nm}$. Bandpass interference filters determine the wavelength interval recorded by each camera. The standard wavelength configuration was used for this mission- $25 \mathrm{~nm}$ bandpass filters centered at $450 \mathrm{~nm}$ (blue), $550 \mathrm{~nm}$ (green), $650 \mathrm{~nm}$ (red), and $800 \mathrm{~nm}$ (near infrared). The CCD dimensions in each camera are $768 \times 576$ square pixels with 8 -bit radiometric resolution. An aircraft altitude of $6115 \mathrm{ft}(1865 \mathrm{~m})$ above ground level (agl) produces a nominal spatial resolution of $1 \mathrm{~m}$ per pixel.

The CAMIS processing unit consists of a 233-MHz Pentium processor with $64 \mathrm{MB}$ of RAM, 23-GB hard drive, and a Matrox Genesis Image Processing Card with 24-MB onboard memory. A Motorola Oncore GT global positioning system (GPS) receiver obtains absolute code phase positions for the center pixel of each image. The system was mounted and flown onboard a Cessna 172 Skyhawk. The pilot navigated to each site and along each flightline using an onboard moving map with code phase GPS.

Geographic coordinates defining the extent of each individual reference are needed to develop a series of flightlines that encompassed the extent of each set of coordinates. As the CAMIS system acquires images as discrete four-band digital frames, flightline calculations are identical to those used for standard aerial photographic missions. Nominal frame-to-frame overlap (or endlap) should be $60 \%$, while nominal flightline sidelap should be at $20 \%$. Data capture occurring within \pm 1.5 hours of solar noon ensures optimal solar illumination and to minimize shadows.

\subsubsection{Poplar Island Restoration}

CAMIS images were acquired on 11 February 2001 between 1100 and 1200 hours. Conditions were sunny with light winds, providing adequate solar illumination and acceptable aircraft attitude during image acquisition. Ten unique reference wetland sites were covered. Figure 2 depicts the completed flightlines over several sites on or near Tilghman Island, Maryland. The orientations of the 
individual overlapping CAMIS frames are shown. During initial project planning, the research staff decided that image acquisition should coincide with low tide conditions. Tidal charts predicted low tide at 1333 hours on 11 February. While this time is approximately 2 hours after local solar noon, the imagery would capture the wetland hydrologic conditions relatively close to low tide.

The desired nominal spatial resolution was one $\mathrm{m}^{2}$ per pixel. This required an aircraft altitude of approximately $6115 \mathrm{ft}(1865 \mathrm{~m})$ agl. However, air traffic control at the Baltimore Washington International Airport (BWI) restricted the aircraft to a ceiling altitude of $5500 \mathrm{ft}(1677.5 \mathrm{~m})$. The airspace above $6000 \mathrm{ft}$ (1830 $\mathrm{m}$ ) is reserved for incoming commercial aircraft. This lower altitude increased the nominal spatial resolution (i.e., decreased the pixel size) to $\sim 0.75 \mathrm{~m}^{2}$ per pixel (0.87- $\times 0.87-\mathrm{m}$ pixel dimensions).

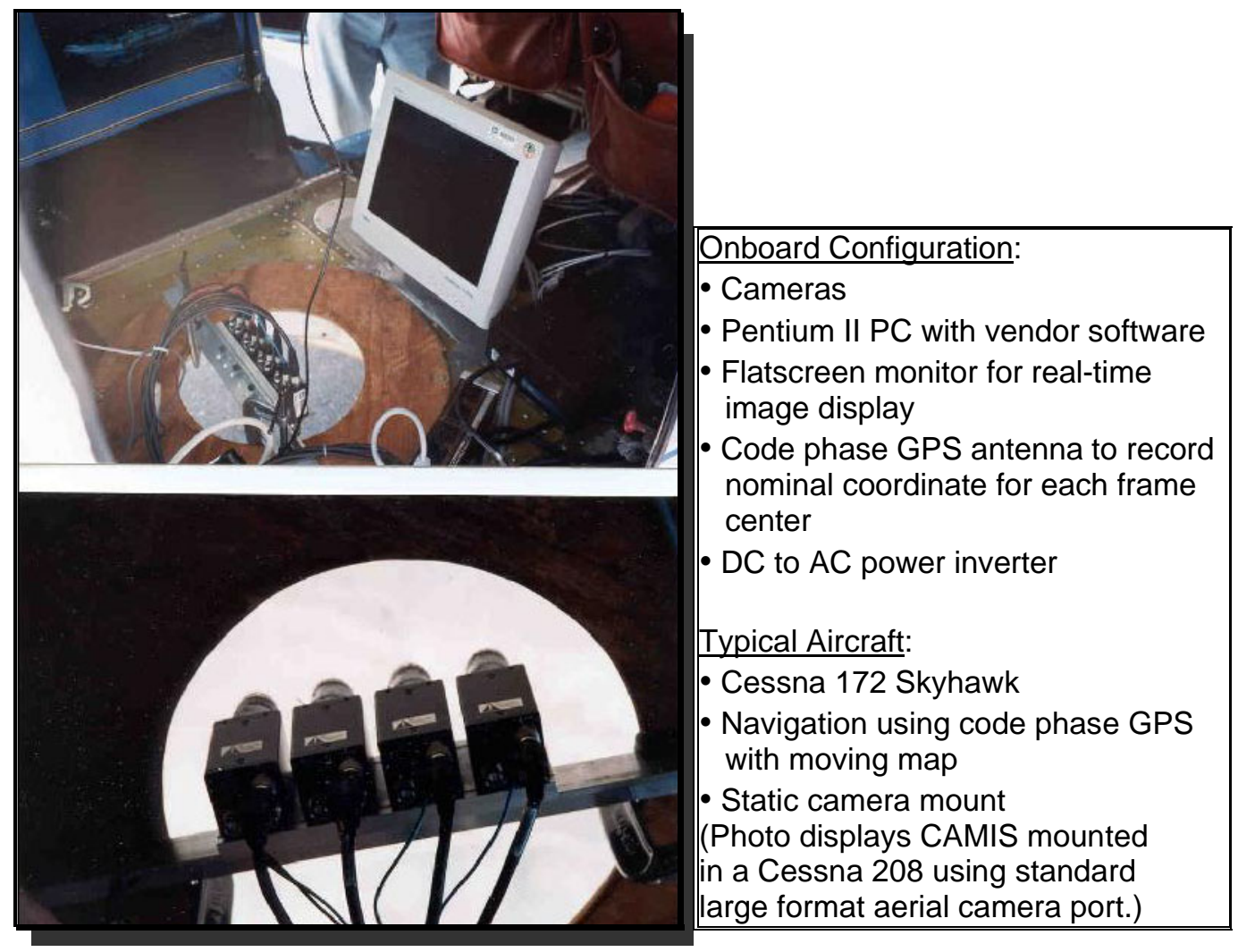

Figure 1. CAMIS model 4768P airborne digital multispectral sensor. 


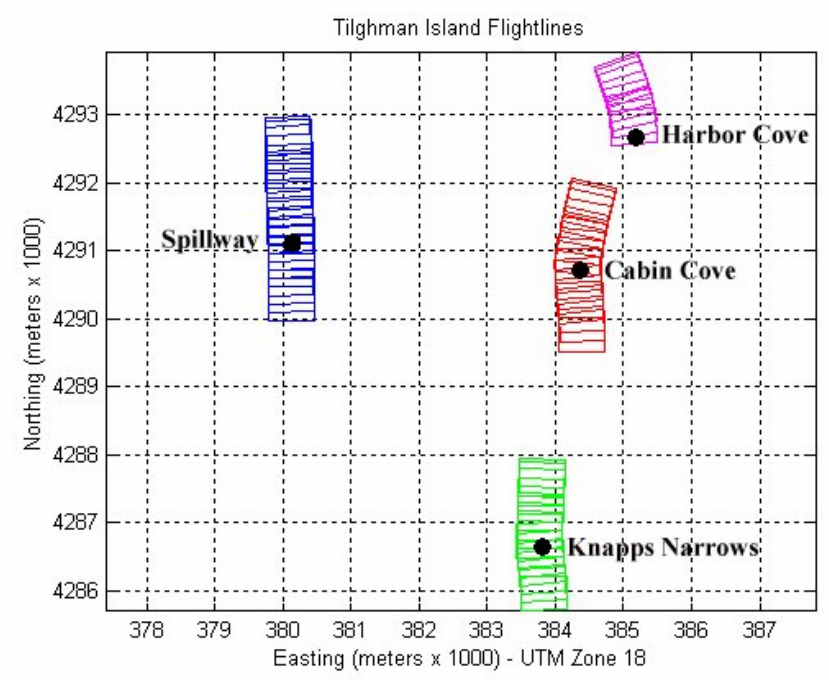

Figure 2. Sample flightlines.

\subsubsection{Levee Inspections}

CAMIS imagery was also acquired for the levee inspection study. Figure 3 shows the orientation and length of the nine flightlines developed during mission planning to cover the Syn Levee. The desired nominal spatial resolution was $1 \mathrm{~m}^{2}$ per pixel. This required on aircraft altitude of approximately $6115 \mathrm{ft}(1865 \mathrm{~m})$ agl.

Images were collected on two dates: 4 September 2001 and 17 July 2002. The first mission was only marginally successful in acquiring cloud-free images. A lingering mid-summer weather pattern resulted in the formation of low-altitude strato-cumulus clouds by 1000 hours every morning during the week of 2 September 2001. Therefore, a second mission was flown in mid-July 2002. Weather conditions were much more favorable, resulting in cloud-free frames of the entire levee. Image acquisition for both missions occurred within \pm 1.5 hours of solar noon to provide optimal solar illumination and to minimize shadows. 


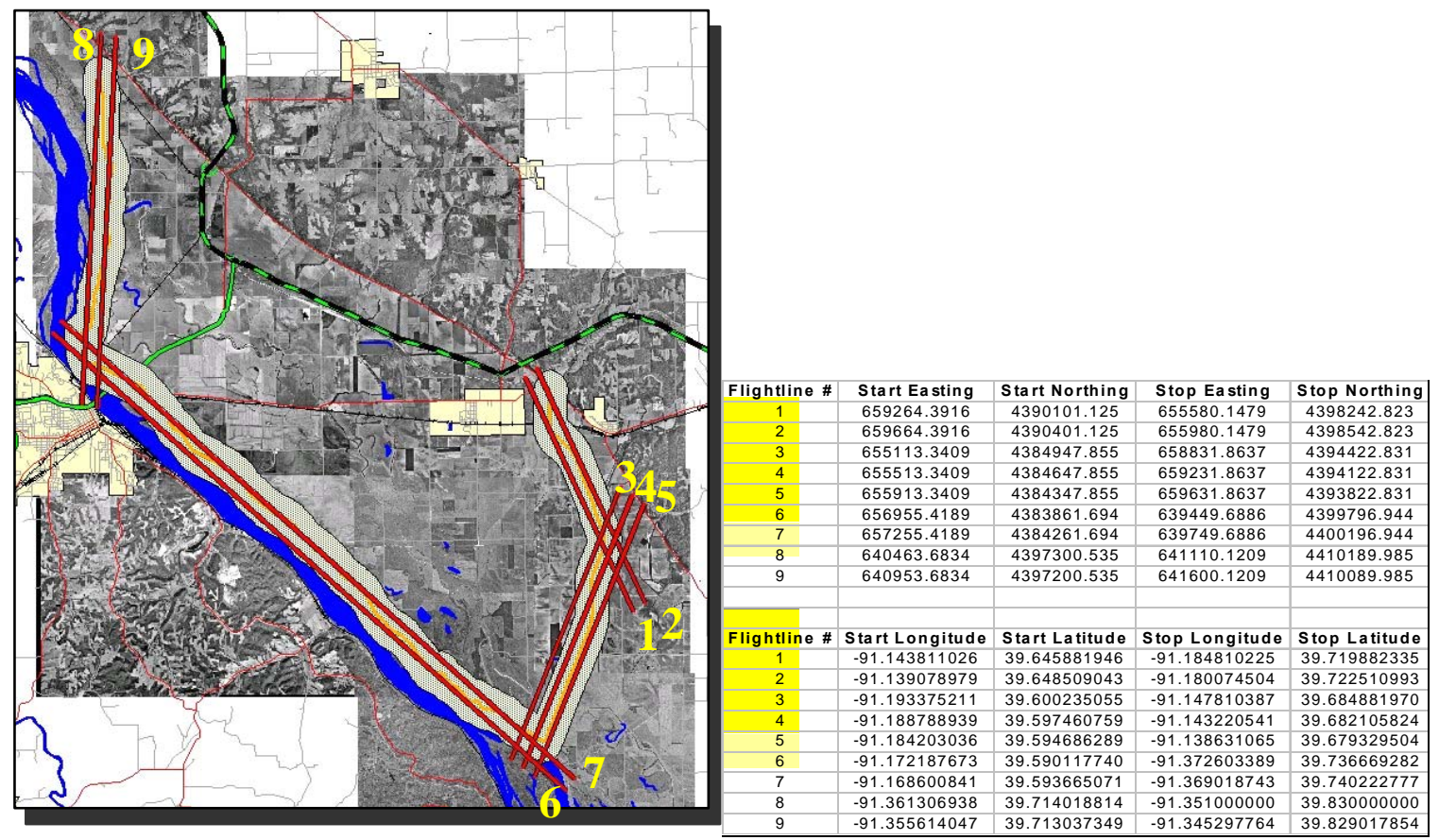

Figure 3. Orientation of the nine flightlines (red) required to acquire a complete CAMIS image set over the Sny levee. The table lists both the UTM and Lat/Long coordinates for the starting and stopping point of each flight path.

\subsubsection{Invasive Species Mapping}

The CAMIS sensor acquired 1-m spatial resolution imagery over several rectangular $(5-\times 2-\mathrm{km})$ sites over Lake Okeechobee on two dates: 31 January 2001 and 9 December 2001. The January 2001 CAMIS data proved to be of little utility to this project. South Florida was experiencing a very dry period and the water levels in Lake Okeechobee were extremely low. During field data collection, it was determined that the distribution of invasive species, either terrestrial or aquatic, was so low that delineation would be impractical with remotely sensed data. Figure 4 shows three CAMIS mosaics built from much higher quality imagery acquired in December 2001. 


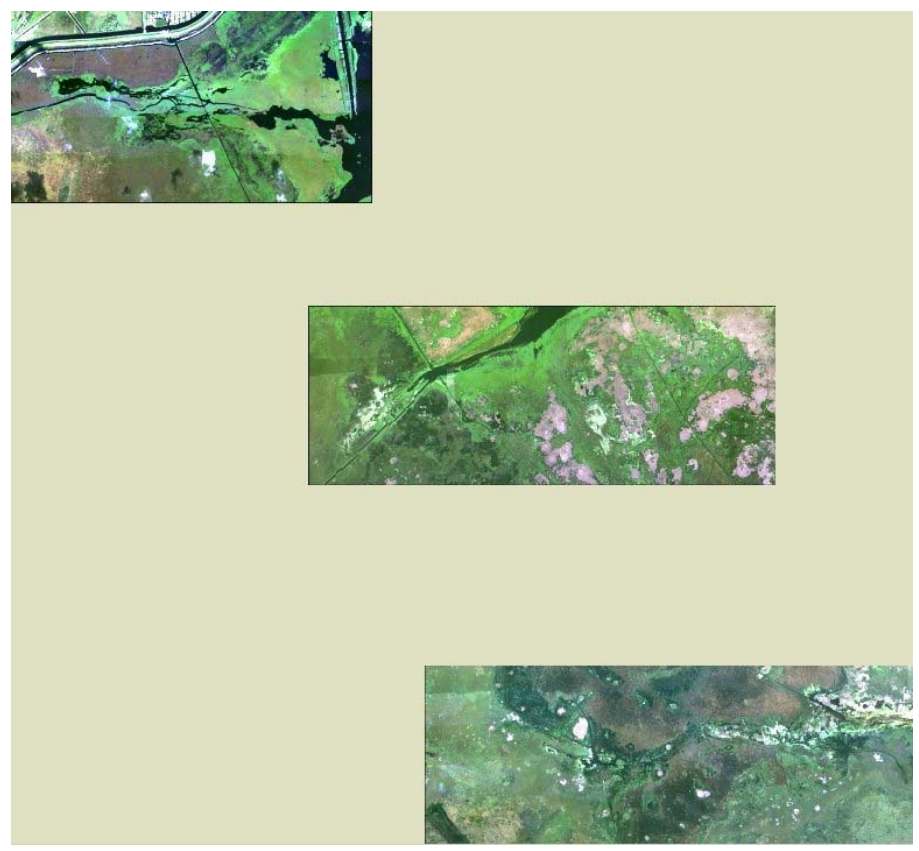

a. True color.

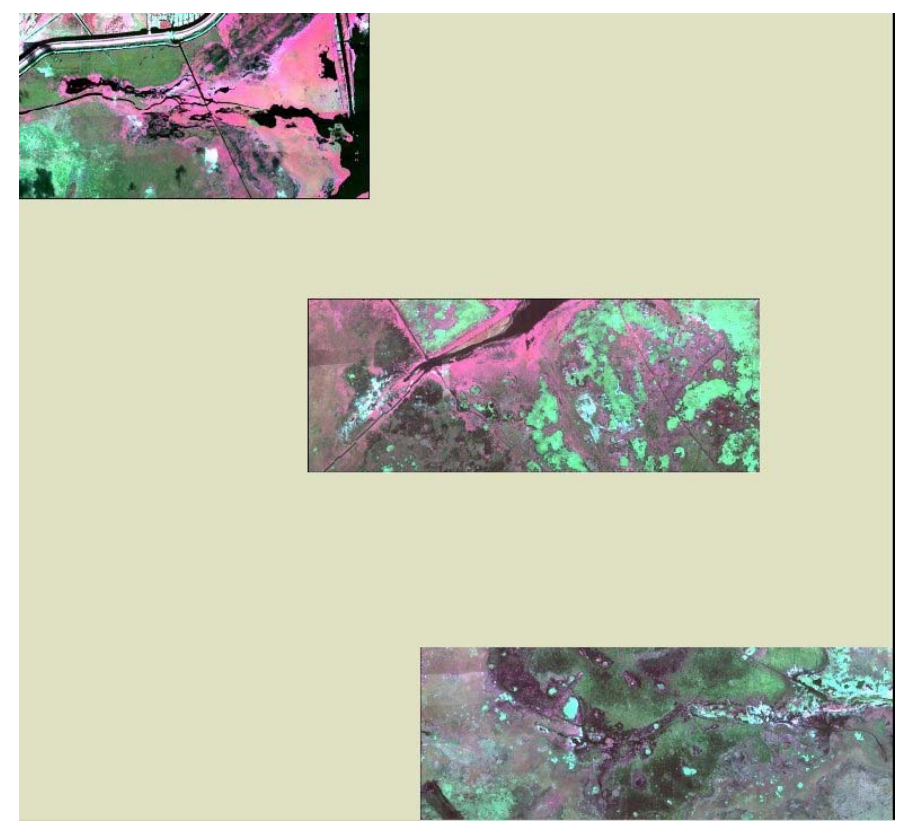

b. False color.

Figure 4. True-color and false color composites of Fisheating Bay, Monkey Box, and Moonshine Bay acquired 9 December 2001. 


\section{$2.2 \quad$ IKONOS}

A multispectral IKONOS satellite image was acquired over a larger area of western Lake Okeechobee, which included the CAMIS study sites, on 17 July 2002. This commercial satellite, operated by Space Imaging (Thorton, Colorado), acquires four-band multispectral imagery with 4-m spatial resolution (Fig. 5). The spectral band widths of the IKONOS imagery are wider as compared to the CAMIS bands, but are centered in the same regions, thus providing blue, green, red, and near infrared data. Also, IKONOS imagery is acquired with 11-bit radiometric resolution, as compared to 8-bit radiometric resolution with the CAMIS data. The commercial satellite data were purchased to compare to the airborne images. The mid-summer collection date also allowed for a seasonal comparison of vegetation signatures with the CAMIS winter images.

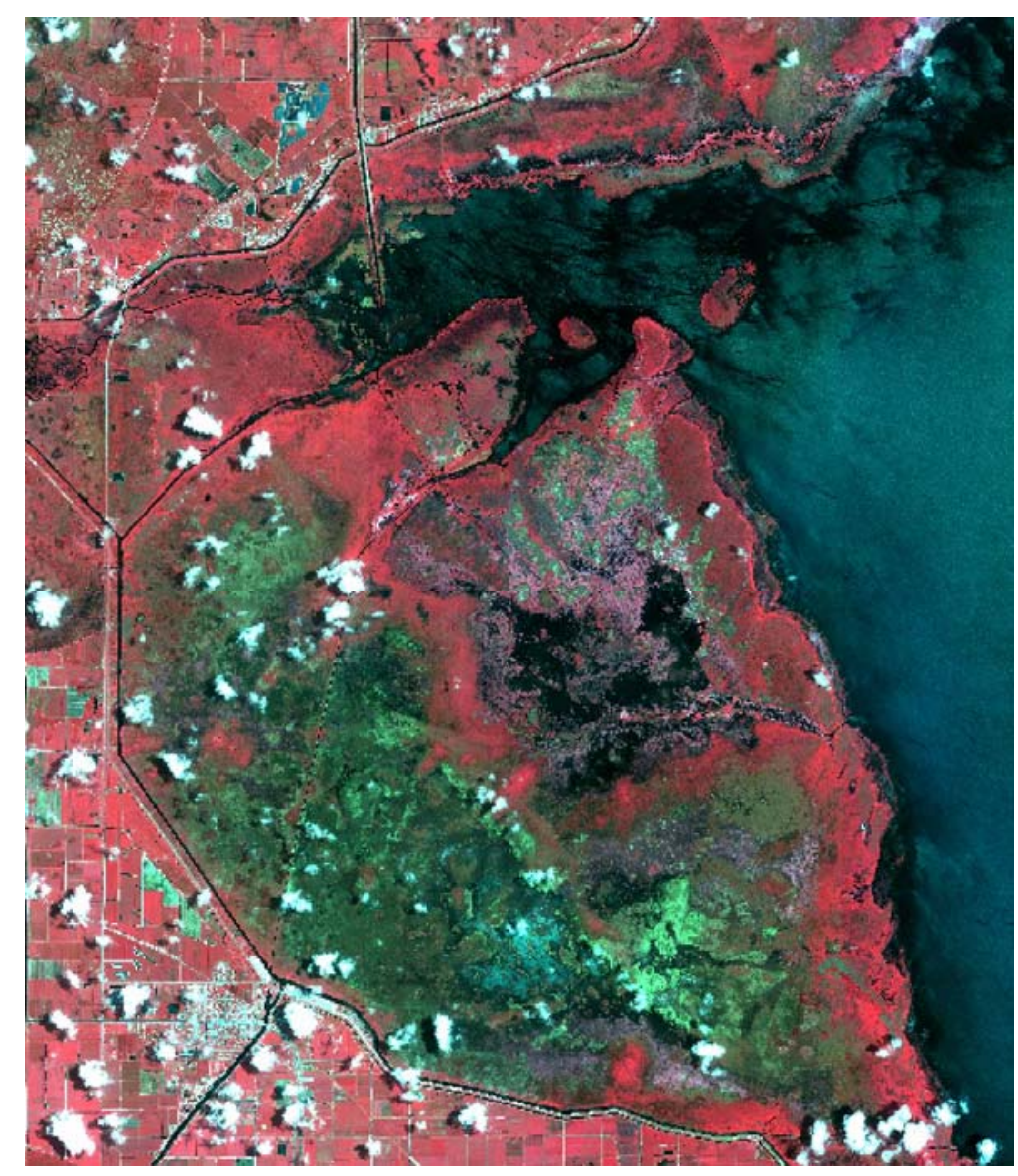

a. False-color composite.

Figure 5. IKONOS image of western Lake Okeechobee, acquired July 2002. 


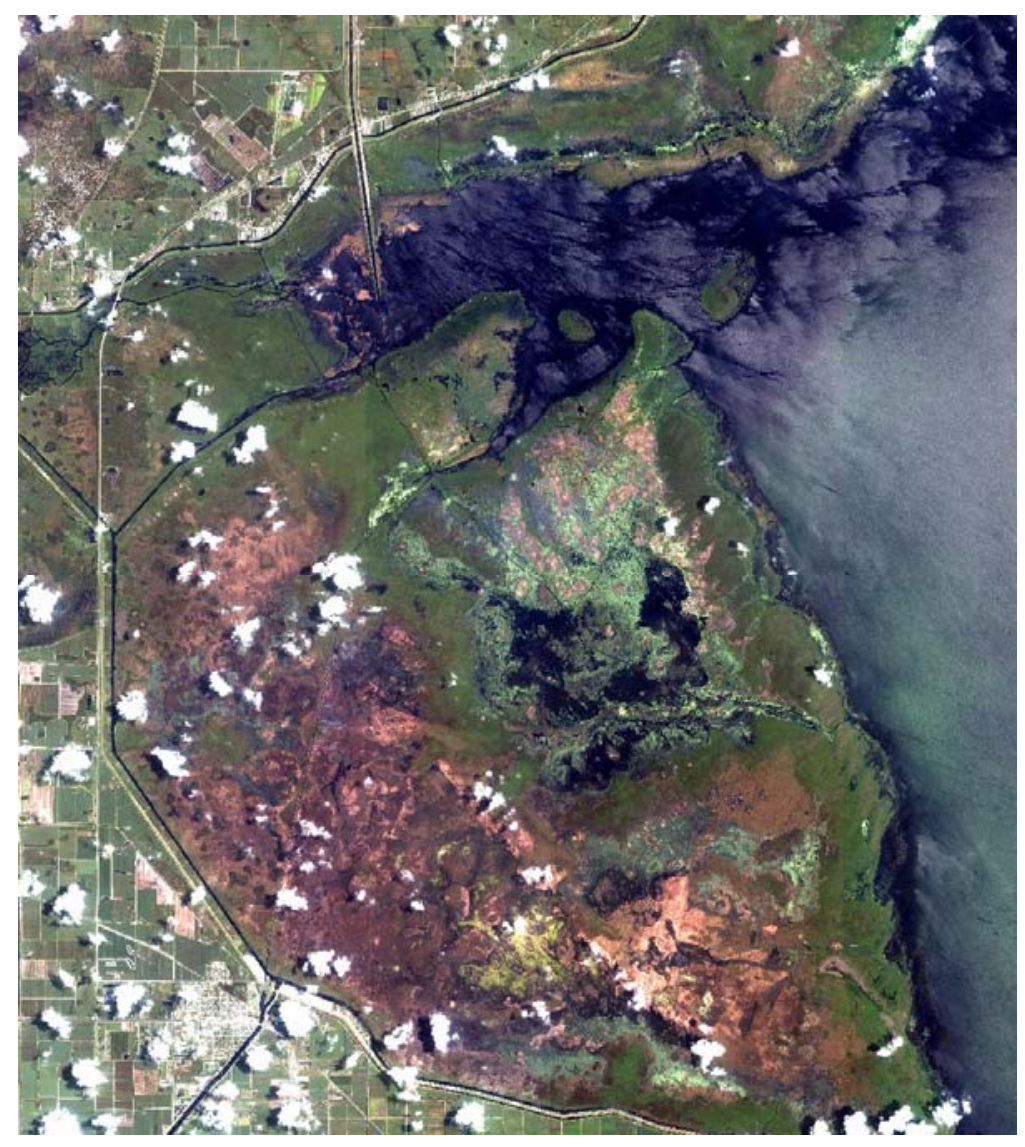

b. True-color composite.

Figure 5 (con't).

\section{$2.3 \quad$ AISA}

Airborne hyperspectral imagery was employed for the detailed wetland vegetation mapping investigation at the Blackwater Wildlife Refuge. The Airborne Imaging Spectrometer for Applications (AISA) is a commercially produced hyperspectral pushbroom type imaging spectrometer. The following system description is adapted from the website of sensor manufacturers: SPECIM*.

\footnotetext{
* Spectral Imaging Ltd., P.O. Box 110 (Teknologiantie 9A), FIN-90571 Oulu, Finland. [http://www.specim.fi/]
} 
AISA is a very compact system, consisting only of two units, the sensor head (front end) and a rugged portable PC with graphical user interface. The image data are stored as a default to a large capacity hard disk.

The basic operation of the direct-vision, dispersing prism-grating-prism (PGP) spectrograph is illustrated in Figure 6. A spectrograph based on the PGP element is composed of a narrow slit, a collimator lens, a PGP element, and a focusing lens (Fig. 6). Radiation entering the spectrograph through the slit is collimated by the first lens and refracted at the prism surface to the right incident angle of the holographic grating. The grating disperses the light according to the common grating equation. Spatial information on the entrance slit is transferred to the image plane at the axis parallel to the slit length direction. The spectrum is formed perpendicular to the optical axis with a good spectral linearity. The tubular direct vision (on-axis) construction leads to low geometrical aberrations in both the spatial and the spectral axes. The optical properties are further guaranteed by using very high quality triplet lenses that are specially designed for imaging.

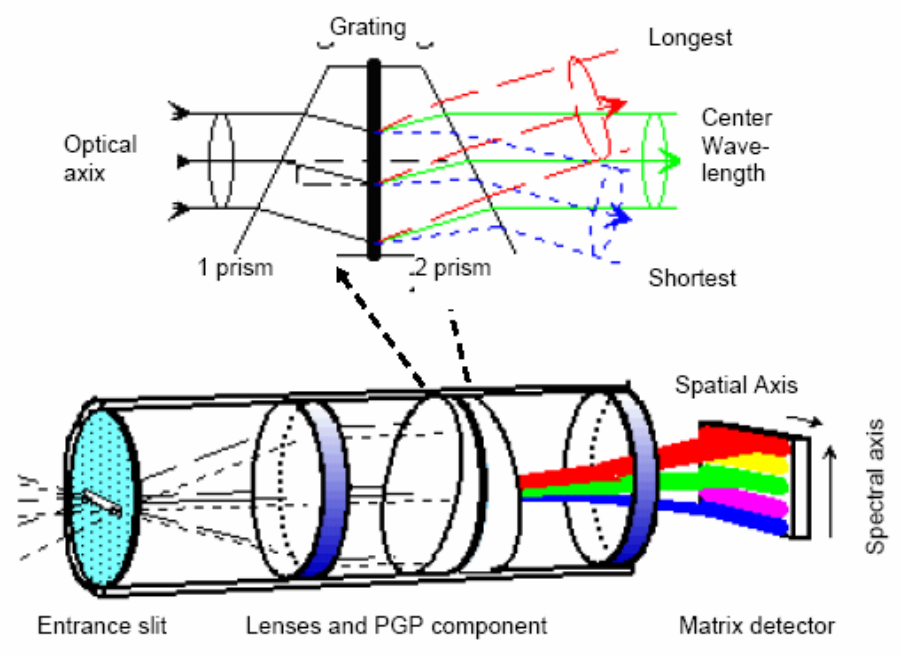

Figure 6. Direct-vision, dispersing prism-grating-prism (PGP) spectrograph used in the AISA sensor.

\subsubsection{Operating Modes}

AISA provides flexibility in its operation mode, depending on user-defined image requirements.. There are four operation modes, which are programmable 
through configuration files. The 384 spatial pixels include 360 ground-target and 20 downwelling irradiance pixels.

Mode A. Provides full spatial (384) and full spectral (288 pixels) information. Neither spectral channel bandwidth modification nor spatial binning is available, but each pixel of the focal plane is digitized as single pixel. Therefore, mode A requires long integration times. This mode is mainly for calibration, testing, and demonstration. However, it can be used in low velocity or high-altitude aerial surveys.

Mode B. Provides full spatial resolution (i.e., smallest pixels) with reduced spectral resolution. This mode offers 384 spatial pixels with no binning, although spatial binning (i.e., summing of 2,4 , or 8 pixels to output larger pixels) is available for low signal level measurements.

Spectral sampling is programmable between 1.5- to 9.4-nm bandwidths within a total wavelength range of $450 \mathrm{~nm}$. Individual spectral bands are programmable. Therefore, the total number of channels is controlled by the selected spectral bands and selected bandwidths.

Mode C. Provides full spectral resolution, with reduced spatial resolution. This mode uses the full hyperspectral capabilities of the sensor (288 bands), but with only 47 pixels in the cross-track direction (as compared to 384 with Mode B). Further spatial binning (i.e., decreased spatial resolution) is still available. Mode $\mathrm{C}$ is suitable for hyperspectral applications that require varied spatial resolutions.

Mode $D$. Provides the greatest variation in both spectral resolution and spatial resolution. While Mode D minimizes the amount of data to be collected and allows high frame rates, it does not offer the highest spatial resolution.

\subsubsection{Downwelling irradiance measurement}

The quality of airborne hyperspectral remotely sensed data is enhanced by using downwelling irradiance measurements together with the actual target measurements. In AISA this is implemented using a hemisphere (or flat) reflector attached to the aircraft roof receiving direct solar radiation. In the best case, there is a clear, sunny sky. The correction is more difficult if atmospheric conditions are cloudy or partly cloudy. The resulting signal is fiber-optically connected to the spectrograph such that it covers a few pixels from the swath width (i.e., a few columns on the detector). Thus, there is a simultaneous measurement of downwelling irradiance with the same wavelength range as is captured by the thermoelectrically stabilized charge-coupled device (CCD). This is a great advantage to systems requiring mechanical movement of the instrument or even a separate 
instrument to measure the signal representing the downwelling irradiance. Naturally, even in the best case, the measurement is only an approximation of the downwelling irradiance on the ground owing to various atmospheric effects.

It was assumed that the vendors used Specim software tools to calculate atsensor-reflectance values for each pixel using the raw radiance images and the downwelling solar irradiance data. The reflectance-calibrated data were then available for analysis and classification.

Hyperspectral imagery was collected over four flights, each flight on a different day. The aircraft maintained an altitude of approximately $3050 \mathrm{~m}$ ( 9842 $\mathrm{ft}$ ) above ground level (agl). The nominal spatial resolution of the data is documented as $3 \mathrm{~m}\left(3-\times 3-\mathrm{m}\right.$ pixels $=9 \mathrm{~m}^{2}$ per pixel $)$.

Parameters set within AISA operating mode B, as described above, produced hyperspectral imagery with 38 spectral bands. The band center for the minimum wavelength was at $461 \mathrm{~nm}$, while the maximum band center was at $891 \mathrm{~nm}$. The width of each of the individual bands varied: bands 1 through 14 maintained a band width of $4.86 \mathrm{~nm}$, bands 15 through 32 maintained width of $5.2 \mathrm{~nm}$, and bands 33 through 38 at $5.32 \mathrm{~nm}$.

The CCD output images were acquired at 12-bit radiometric resolution. Therefore, assuming a minimum digital number (DN) value of 0 (i.e., no reflected electromagnetic radiation detected by the CCD), the maximum DN in the raw imagery during acquisition was theoretically 4095 . After reflectance calibration, the DN's were transformed to a signed 16-bit radiometric quantization. Atmospheric conditions during image acquisition were not documented. The images show very few clouds; therefore, it is assumed that skies were clear.

The majority of the images were acquired in either an east-to-west or a westto-east flightpath. Several ancillary flightlines were acquired using either north or south flightpaths. Figure 7 depicts each flightline and its default filename. In general, the east-west lines are numbered from 1 to 20 , with path 1 at the north extreme of the study site and path 20 at the south. It is assumed that the trailing character in the filename (i.e., E, W, N, or S) indicates the direction the aircraft was flying during acquisition. 


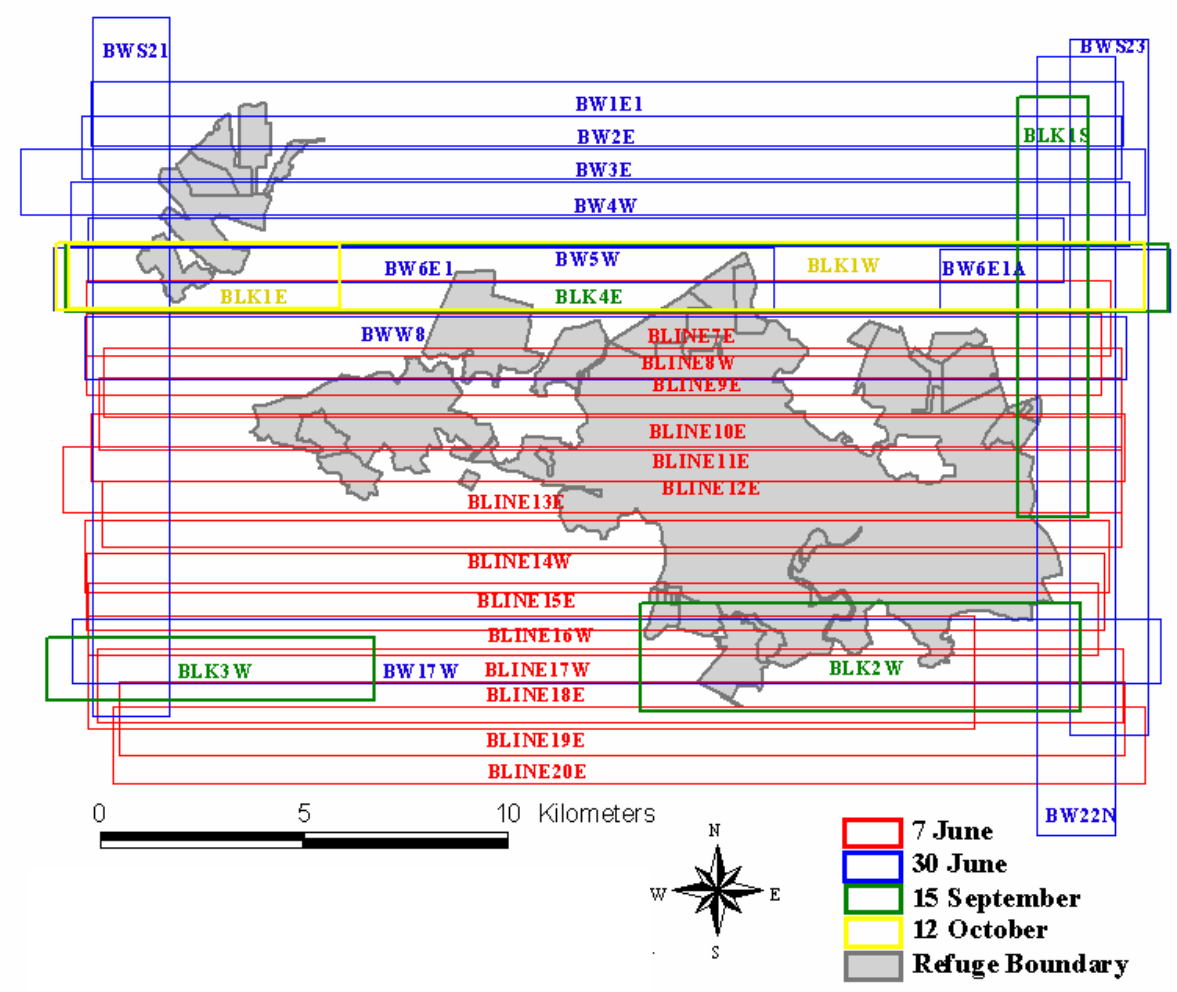

Figure 7. Location and orientation of AISA flightlines over Blackwater Wildlife Refuge. Legend shows acquisition dates. 


\section{IMAGE POST-PROCESSING}

\subsection{CAMIS}

The same post-processing algorithms were used in both the Poplar Island and the Sny levee projects. These techniques are used to correct both radiometric and geometric image distortions. The steps include:

- Band-to-band registration (a geometric correction).

- Removal of frame edge darkening and frame center hot spots (a radiometric correction).

- Frame-to-frame mosaicking (a geometric correction).

- Mosaic geometric registration (a geometric correction).

\subsubsection{Poplar Island}

Degradation of the radiometric (or color balance) quality of digital imagery is the result of influences from a variety of external and internal parameters. External parameters include: solar azimuth angle, solar zenith angle, atmospheric conditions (e.g., aerosols, water vapor), and surface bi-directional reflectance factors. Typical internal parameters that degrade image radiometric quality include lens distortions, lens field darkening, CCD anomalies, and system operation error (e.g., f-stop settings, shutter speed).

Software developed at TEC radiometrically corrected the complete set of CAMIS images. The first step was to band register each frame. Because the four cameras are aligned in sequence, with each lens sited parallel to the others, each band of a single frame has its center, or principal point, offset from the other bands. Figure 8 shows a single false color CAMIS frame before and after bandto-band registration.

The next post-processing step applied a single algorithm to address several of the external and internal parameters that influence radiometric fidelity. Figure 9 displays the visual impact of the correction algorithm on a true color composite. Typical radiometric distortions appear as a general darkening towards the edges of the frame and a bright area (hot spot) at the center. The same brightness shifts are present within most aerial photography, particularly the pronounced hot spot, or glaring, at the center of the photo. 

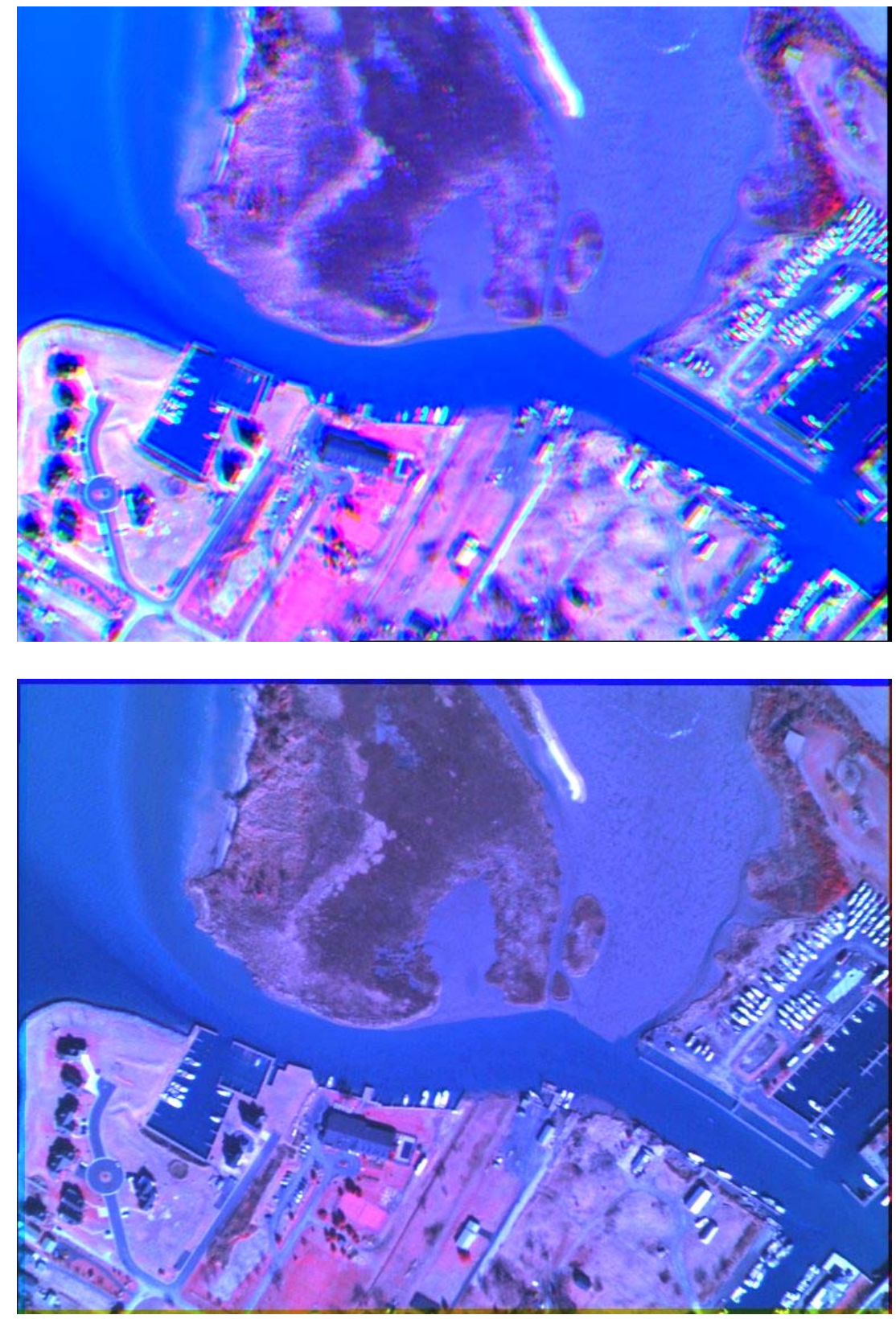

Figure 8. Raw false color CAMIS frame (top) and band registered false color CAMIS frame (bottom) Knapps Narrows, frame 106. 

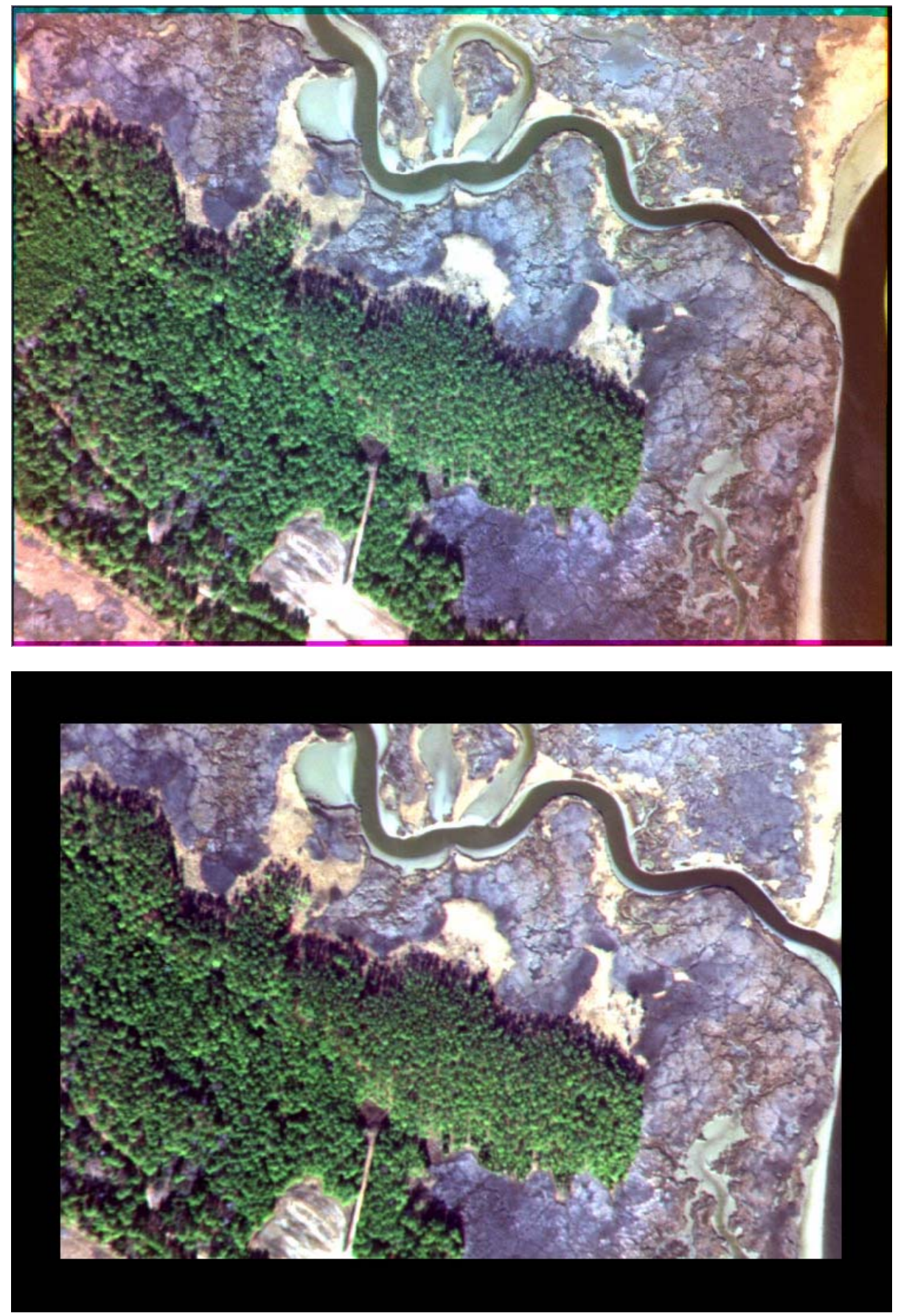

Figure 9. Band registered true color CAMIS frame (top) and radiometrically corrected true color CAMIS frame (below), Hell Hook Marsh, flightline 2, frame 108. 
The corrected CAMIS frame shows greatly improved color balance throughout the entire image (Fig. 9).

The next step was to create image mosaics encompassing each reference wetland. Commercial image processing software (ENVI) was used to mosaic adjacent digital frames. Figure 10 displays a mosaic of three overlapping images covering the PIN (Piney Creek) site. Geometric distortions were minimized by employing a rotation, scale and translation (RST) registration algorithm. In Figure 10, frame 107 served as the base image. Adding frame 106 to the bottom of frame 107 created the initial mosaic. Adding frame 108 to the top of the previous mosaic created the final three-frame composite.

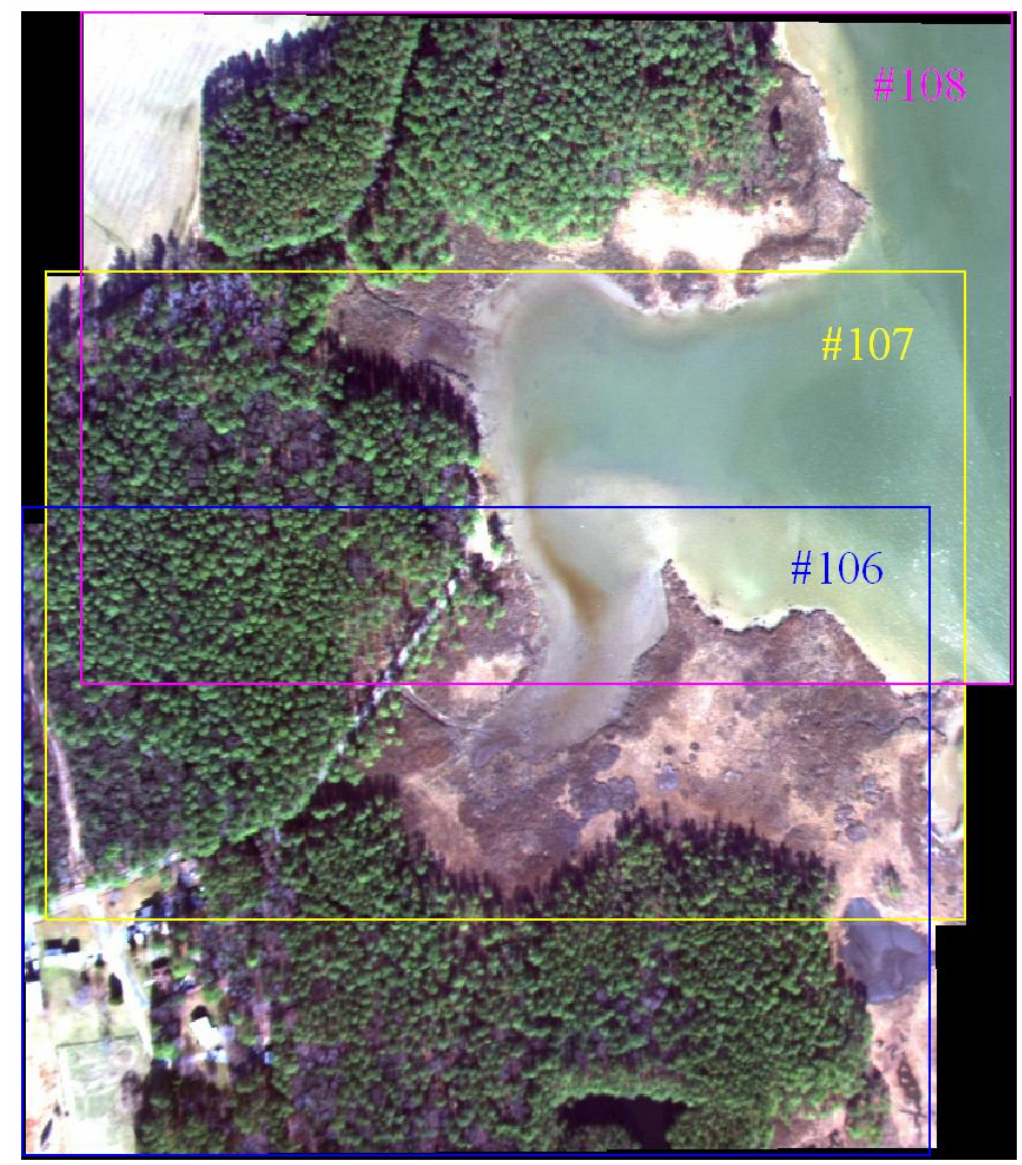

Figure 10. True color, three-frame mosaic of Piney Creek (PIN) wetland. 
The next step was to geographically register each mosaic to real earth coordinates. Commercial image processing software (ERDAS Imagine) geo-registered each individual mosaic to one of two digital base images. The primary source used as digital base maps were high resolution U.S. Geological Survey (USGS) Digital Orthophoto Quarter Quadrangles (DOQQ). The Root Mean Square Errors (RMSE), or registration accuracies, of the small mosaics covering the wetland Knapps Narrows, for example, were less than one pixel $(<0.87 \mathrm{~m})$. The RMSE for the much larger Hell Hook mosaic was roughly nine pixels $(\sim 7.83$ $\mathrm{m})$. Registration accuracy is critical to the overall quality of the final map products. With good positional accuracies of the mosaics, the digital outputs can be co-registered with other data sets, including any available field data, and utilized in subsequent reference wetland investigations.

DOQQs were not available for Piney Creek. Therefore, medium resolution digital 7.5' topographic quadrangles served as base imagery for geo-registering this site. Owing to the decreased level of detail seen in the digital topo quads (e.g., generalized shore lines, generalized road locations), the registration accuracies of the Piney Creek mosaics were much poorer. RMSEs were closer to 10 pixels. Figure 11 shows the relatively minor spatial displacement of the Knapps Narrows mosaic after geo-registration to a DOQQ. Figure 12 shows the significant warping of the Piney Creek mosaic after registration to the digital topographic quadrangle.
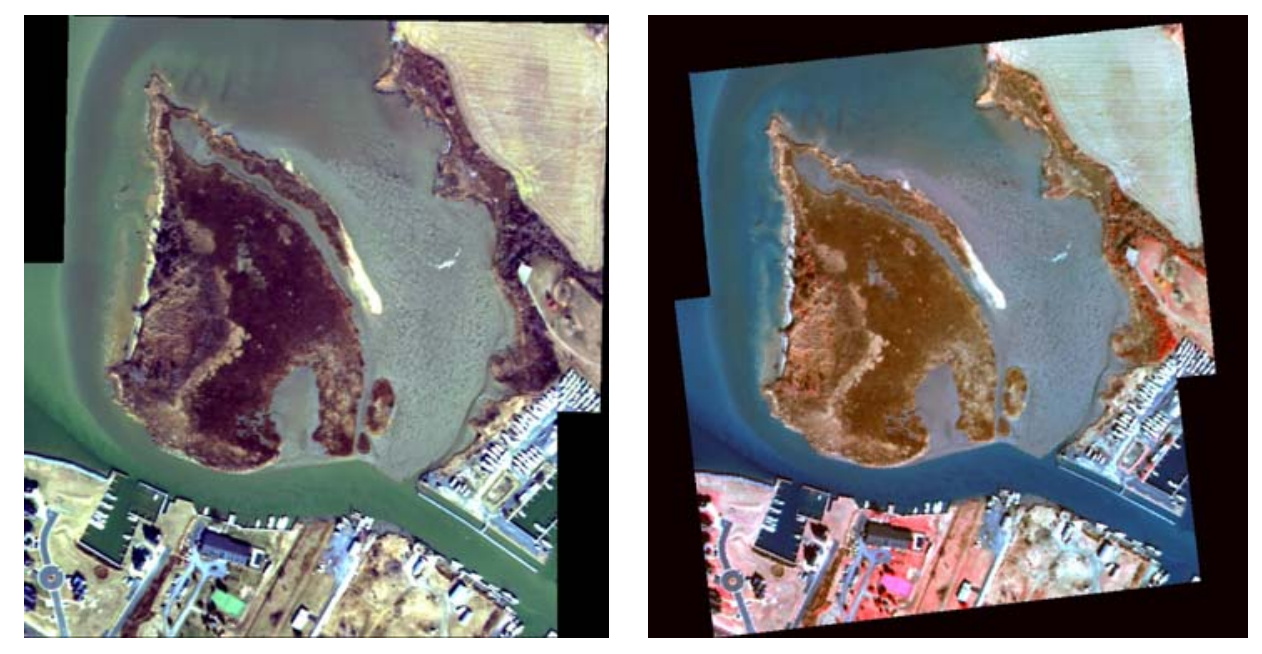

Figure 11. Knapps Narrows mosaic before and after geometric registration to $D O Q Q$. The non-registered mosaic (left) is depicted using true-color (bands 3, 2, and 1); the geo-registered mosaic (right) is depicted using false-color (bands 4, 3, and 2). 

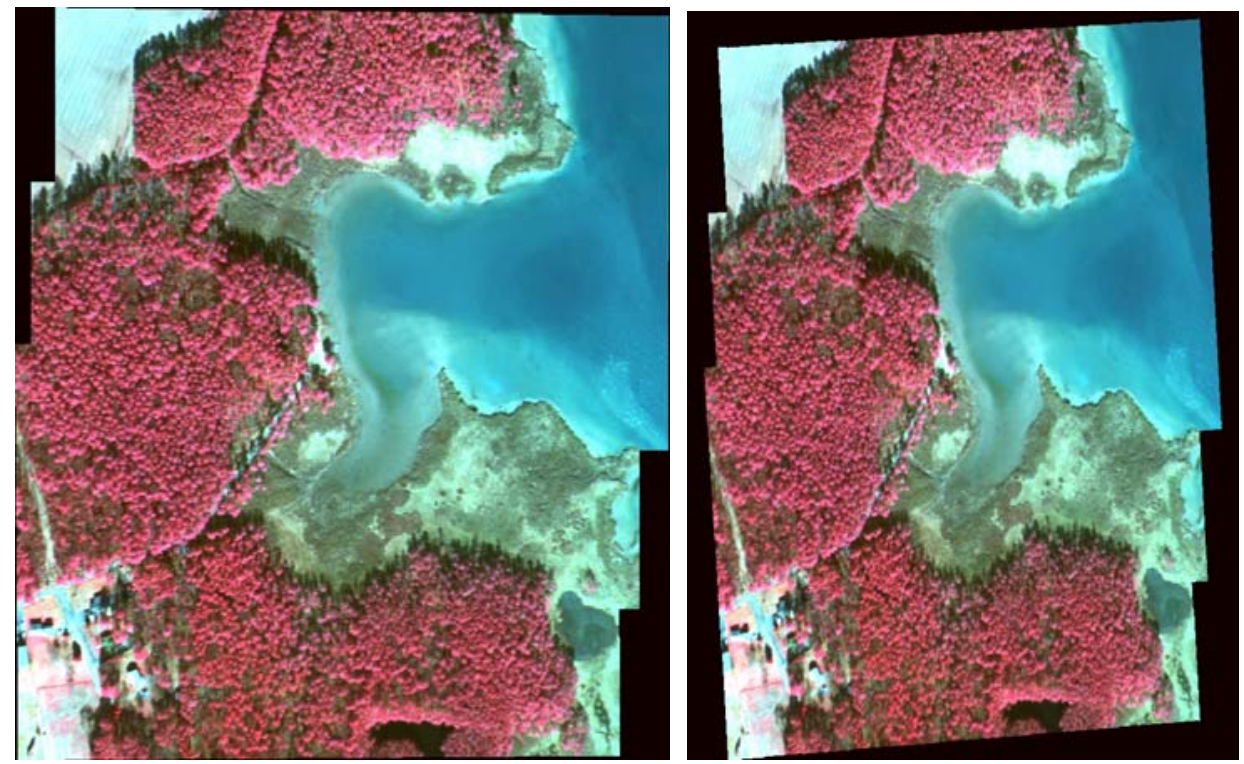

Figure 12. Piney Creek (PIN) mosaic before (left) and after (right) geometric registration to digital $7.5^{\prime}$ topographic quadrangle.
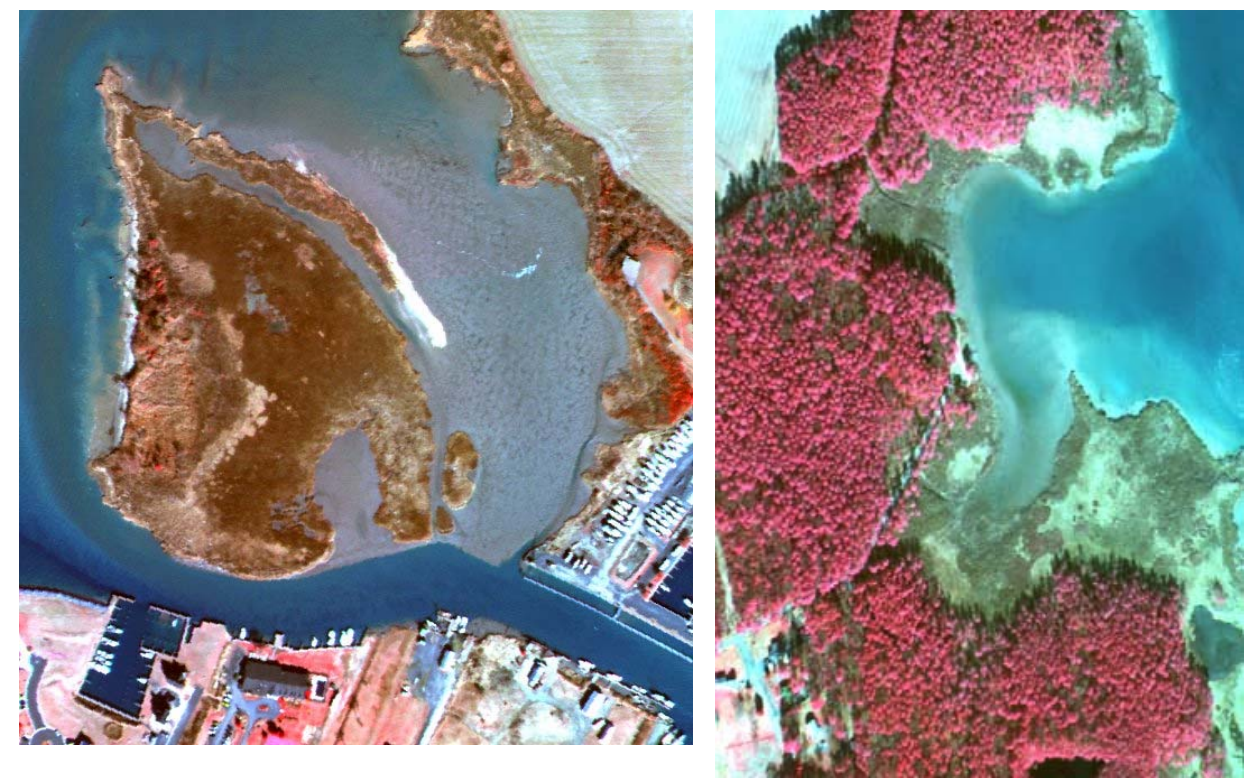

Figure 13. Knapps Narrows and PIN mosaics after subsetting (or clipping). 
All images were registered to Zone 18 of the Universal Transverse Mercator (UTM) coordinate system. Map coordinate units are meters, and the datum defined in the World Geodetic System 1984 (WGS 84).

The final step in preparing the individual mosaics was to clip out a rectangular image. This subsetting operation removes the majority of the background (i.e., black or blank) pixels around the uneven borders of the mosaics. Figure 13 shows the clipped, or subsetted, mosaics for Knapps Narrows and Piney Creek.

\subsubsection{Enhanced Levee Inspections}

The band-to-band registration procedure worked well for both acquisition dates. The radiometric correction algorithm worked poorly for the 2001 acquisition date owing to excessive clouds and cloud shadows. The resulting images showed significant shifts in the intensity and hue of colors when comparing overlapping frames. The 2002 images showed excellent color balance along flightlines after application of the radiometric correction technique.

The frame-to-frame mosaicking step created the initial multi-image mosaics within their own "pixel space." This process was completed manually using the ENVI image processing software. Visually interpreted control points are employed to "stitch" together overlapping images. Therefore, as the mosaics were built by adding one frame at a time, the $\mathrm{X}$ and $\mathrm{Y}$ coordinates were assigned to each pixel as the row and column numbers of the expanding digital file. This frame-to-frame registration procedure also used the RST geometric transformation to sequentially combine the overlapping images. The use of the RST transformation is important to maintain the rectangular shape of each individual frame during the construction of the "pixel space" mosaics. This method of frame-toframe mosaicking is extremely labor intensive. However, the image analyst has complete control over the geometric fidelity of the initial mosaics.

After completing the preliminary mosaic for a section of levee (e.g., flightlines 1 and 2), the next step was to assign real world coordinates to the linear image. The mosaics were geometrically registered to the panchromatic orthophotos. First, second, and sometimes third order polynomial transformations were employed to register each mosaic. This second, and final, geometric registration step was also labor intensive. The 2001 mosaics were poorly registered to the orthophotos. The poor output from the geometric registration was attributable to the linear nature of the pixel space mosaics. Significant warping (i.e., distortion) was observed at the ends of the flightlines (Fig. 14). Results were improved for the 2002 images by geo-registering smaller clusters of four to five frames to the orthophotos (Fig. 14). 

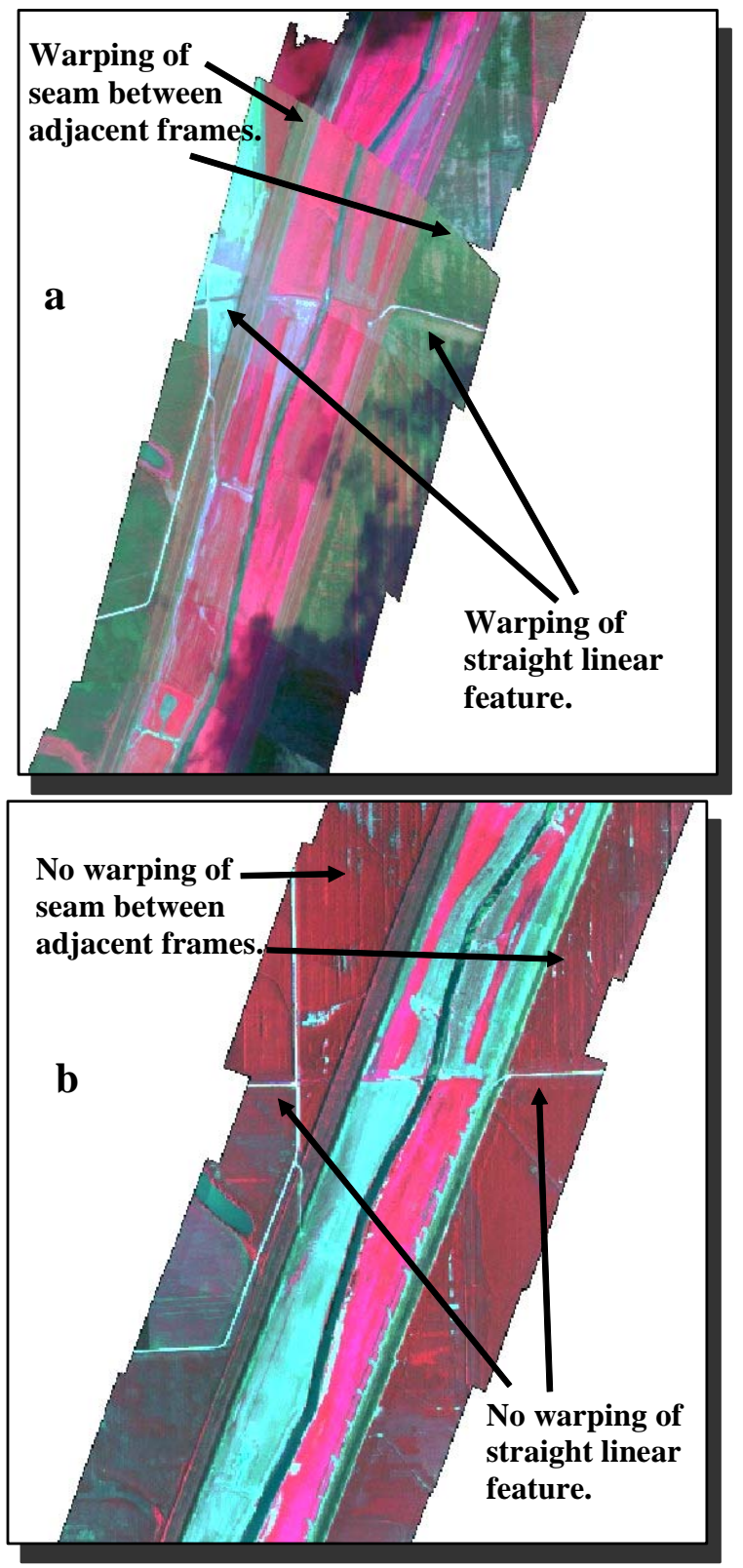

Figure 14. Examples of (a) poor, and (b) good geometric registration.

The frames omitted from lines 6 and 7 are ascribable to excessive cloud cover. Flightlines 8 and 9, however, are cloud-free and show better radiometric balance. The July 2002 imagery shows generally good radiometric balance with only one small area of cloud shadows near the center of flightlines 6 and 7. 
Approximately 160 CAMIS frames were used in the creation of each mosaic (Fig. 15 and 16).

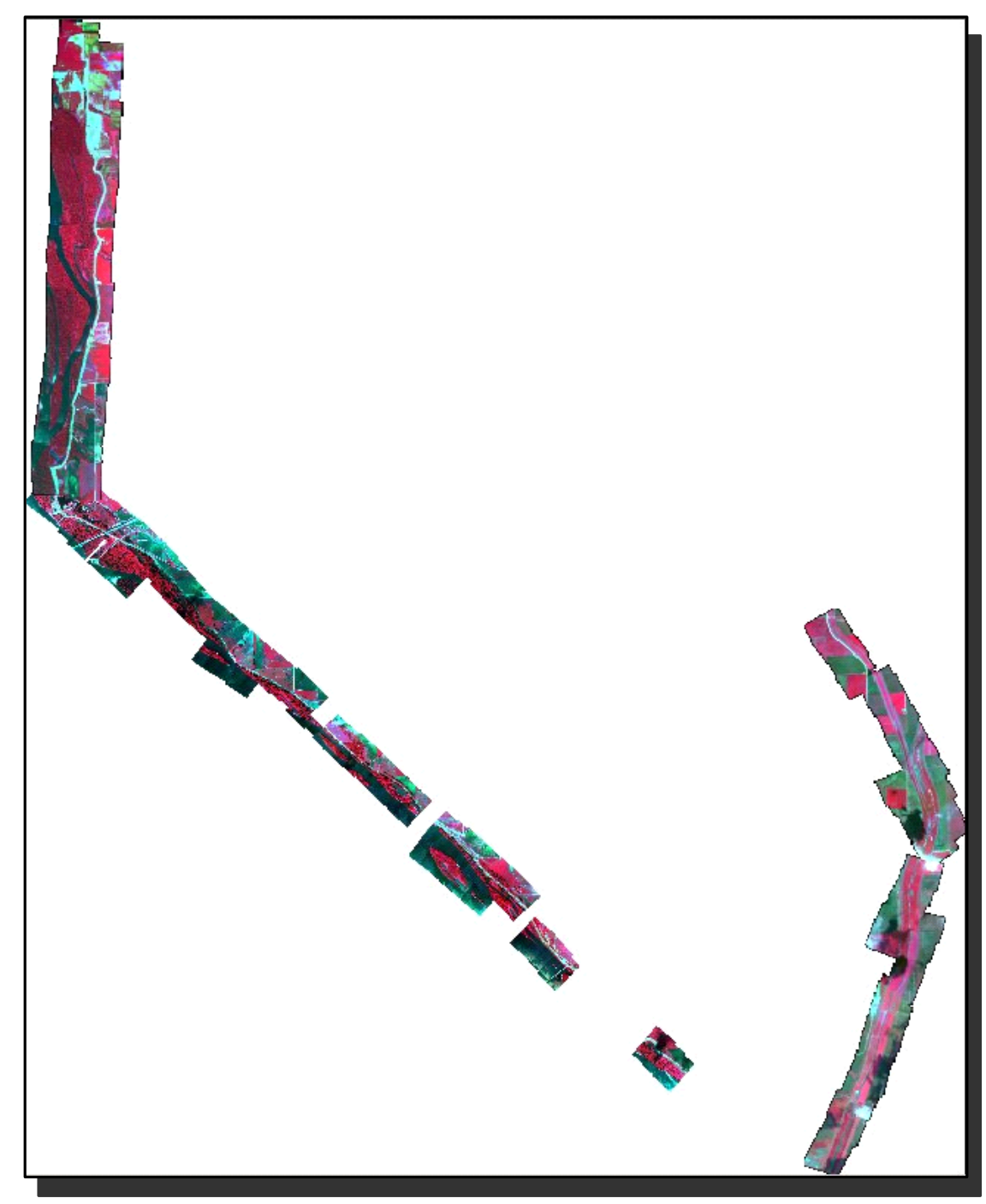

a. False color.

Figure 15. 4 September 2001 geo-registered mosaics. 


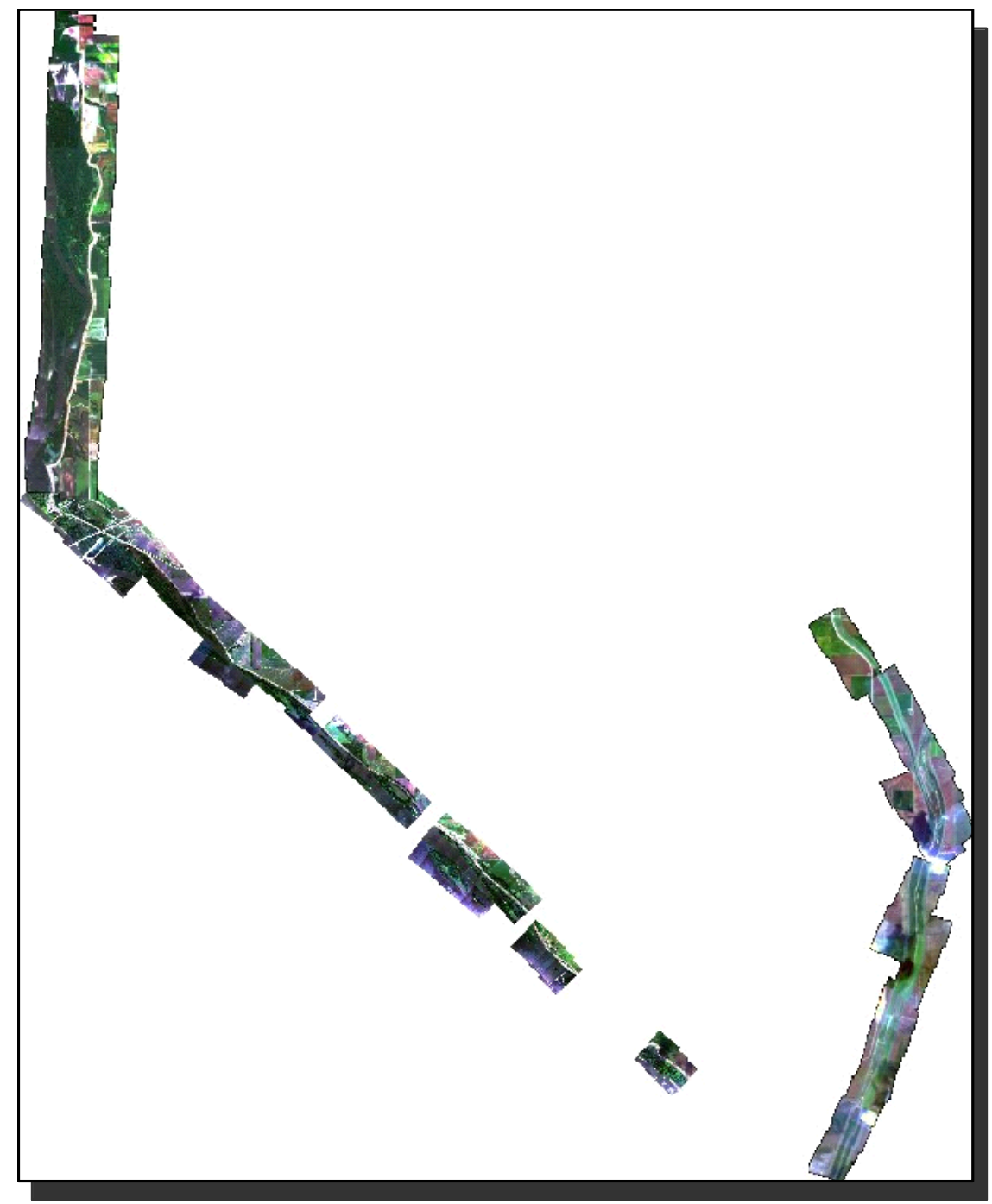

b. True color.

Figure 15 (cont'd). 


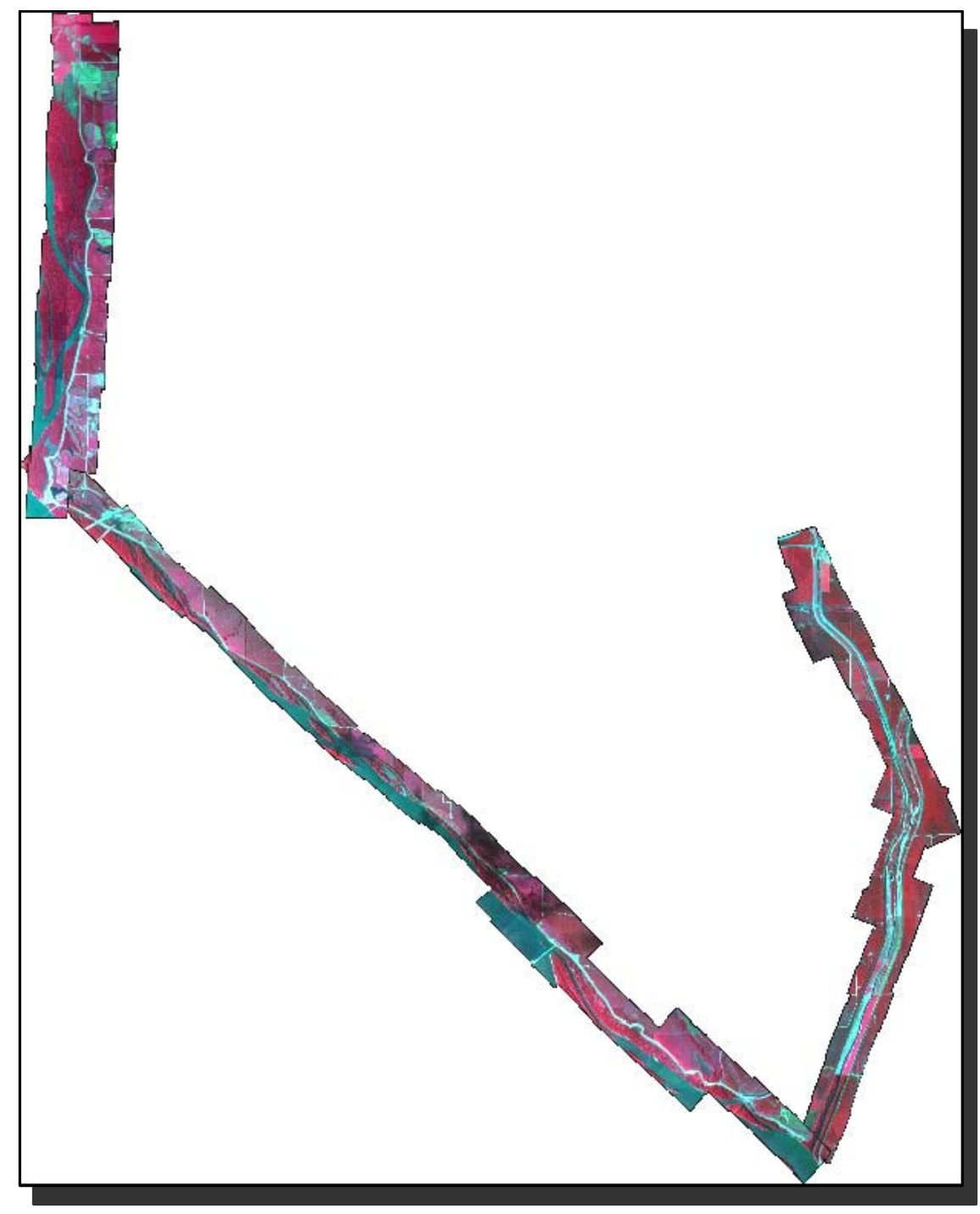

a. False color.

Figure 16. 17 July 2002 geo-registered mosaics. 


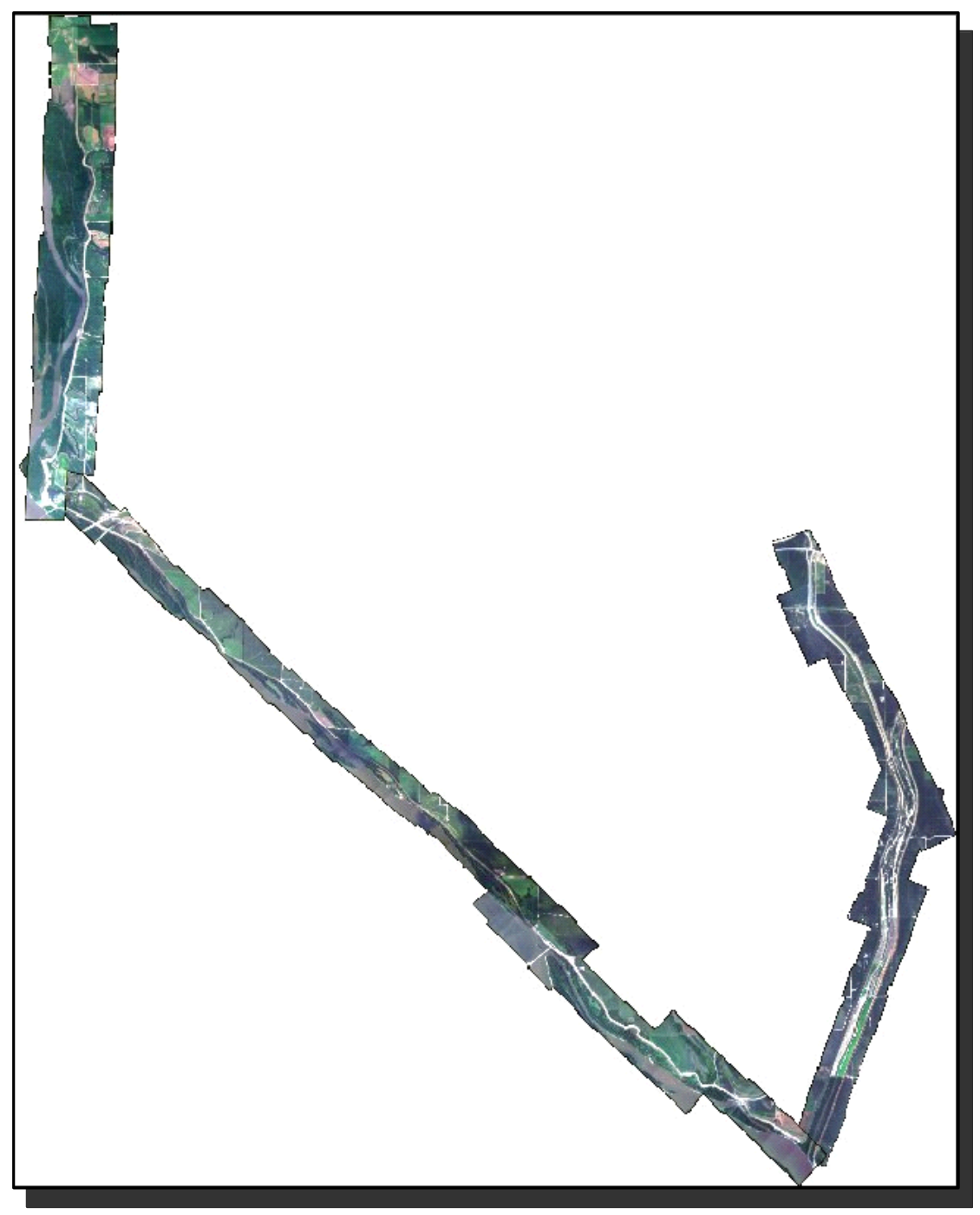

b. True color.

Figure 16 (cont'd). 


\section{$3.2 \quad$ IKONOS}

The primary post-processing step applied to the IKONOS image was to reproject its coordinates to the NAD27 datum. Additional steps included:

- Masking out (i.e., removing) cloud pixels.

- Clipping (i.e., removing) the area outside the Lake Okeechobee levee.

- Rescaling the 11-bit data to 8-bit data.

\subsection{AISA}

The reflectance data were provided on a set of nine CDs. Each flightline included a suite of files including a reflectance calibrated image file (*.dat), an ENVI formatted header file, a sensor information/calibration file, an instrument calibration file, and an aircraft navigation file.

\subsubsection{Geometric Correction}

The raw reflectance data for each flightline were unzipped and individually displayed to ensure the images were not corrupt. Based on the development of the flightline diagram (Fig. 7), combined with an assumption of radically different vegetation phonologies that would be observed in June as compared to September and October, post-processing of the AISA images was limited to the 7 and 30 June data.

Several test mosaics of a two to three adjacent flightlines, using only a subset of bands to minimize processing time, were created to evaluate the geometric fidelity of the AISA imagery. The mosaics showed acceptable georegistration of the reflectance-calibrated images, with horizontal errors no greater than three to four pixels. Certainly a higher level of geometric accuracy would be desirable. However, considering the volume of data acquired and the intended purpose of the imagery, this level of geometric accuracy was considered to be acceptable. Figure 17 shows two examples of the typical departure of linear features between adjacent flightlines. 


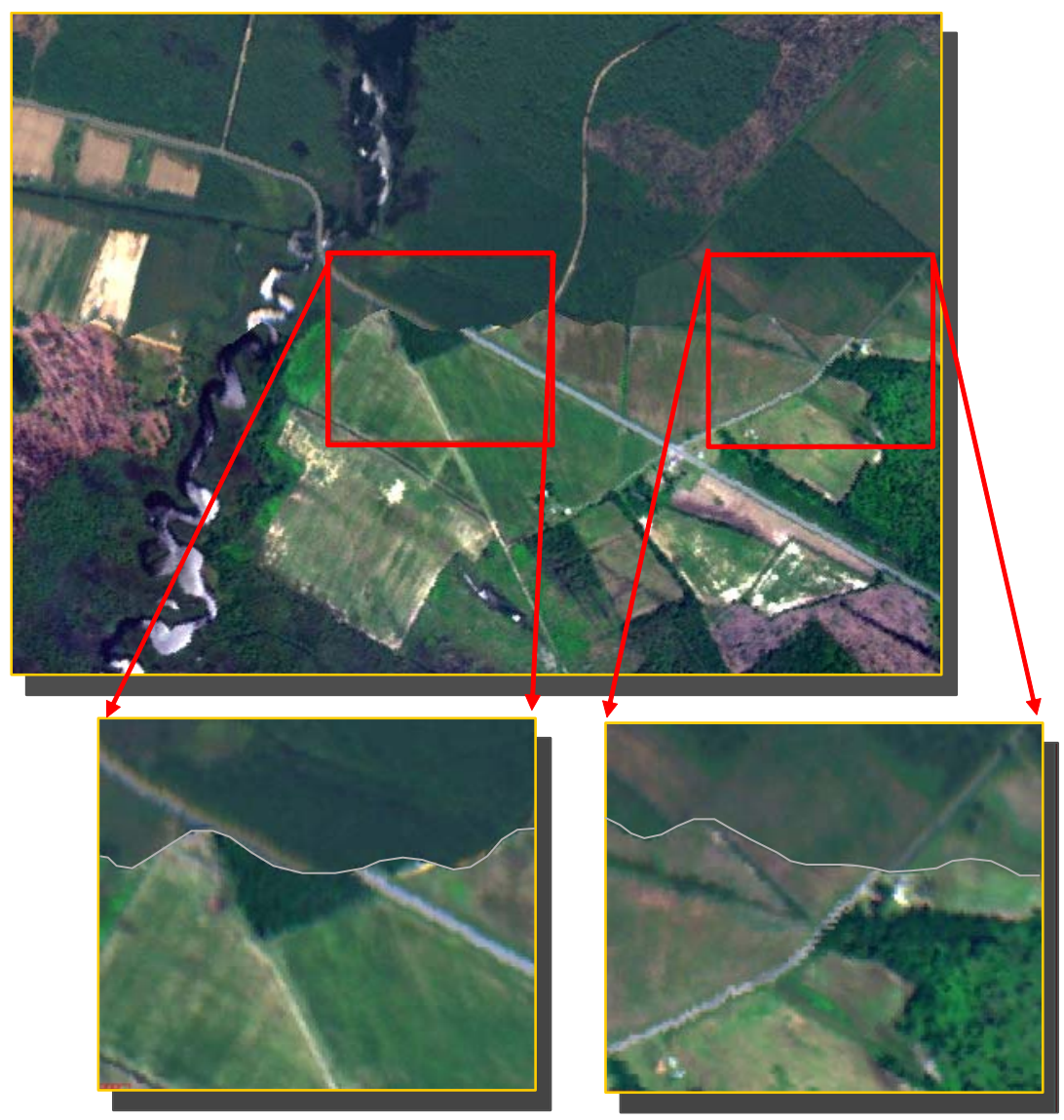

Figure 17. Examples of the geometric quality of the AISA imagery. The vendor provided no quantitative assessment of the actual error in registration. A qualitative evaluation suggests that the geo-rectification processes used to rectify the AISA flightlines are adequate for this application. The gray line, within the insets, defines the boundary (or seam) between the two adjacent flightlines. The area shown is along Route 336 north of Crapo.

\subsubsection{Radiometric Correction}

3.3.2.1 Histogram Evaluation and Adjustment. During the construction of the preliminary mosaics, the radiometric fidelity of adjacent flightlines was observed to be relatively poor. Figures 18 and 19 show the radiometric departure between two adjacent flightlines. It was clear that some form of radiometric normalization or equalization would be needed to minimize the dramatic brightness shifts between overlapping images. At the beginning of this research effort, we assumed 
that little if any radiometric corrections would be required, given that the hyperspectral imagery was calibrated, or corrected, to at-sensor-reflectance values.

This suggests that the identical land surface features (e.g., dominant loblolly pine crowns, soybean fields at the same stage of development) should have identical, or nearly identical, DNs across all flightlines acquired on the same Julian Date. If the land surface feature signatures were equal across flightlines after the reflectance calibration, adjacent images (e.g., BLINE7E and BLINE8W) would produce nearly seamless hyperspectral mosaics. This was not the case with the Blackwater images. Consistently, the seam between two adjacent images was visually detectable. Radiometric normalization or equalization, involving manipulation of the image histograms, was employed to radiometrically match overlapping flightlines.

Histogram analyses generally involves both statistical and graphical tools, as well some general understanding of the anticipated minima, maxima, and distribution of possible data values (i.e., DNs) within an image-based data set. Nearly all 38 bands from two overlapping flightlines-BLINE7E and BLINE8W-show a minimum value of 0 , corresponding with the background pixels within each image file. Raster data, such as digital imagery, must be stored in matrix (i.e., rectangular) file formats. Therefore, with non-rectangular output image types, such as AISA data acquired using a pushbroom scanner system, a rectangle with dimensions (and accompanying geographic coordinates) slightly larger than the actual image is created to store the data. The empty, or blank, pixels surrounding the image data become background pixels, and are generally assigned an output DN of 0 . From an image processing perspective, background pixels add to the overall complexity of the image statistical characteristics. Figure 20 shows flightlines BLINE7E and BLINE8W and their associated mean reflectance spectra, including the background pixels. It is good practice to, when possible, remove background pixels by trimming the original file using a slightly smaller rectangle. Some data pixels will likely be lost during the resizing of the file. If the pixels to be clipped are critical, removing the background pixels may not be possible. Such was the case with the AISA images. The amount of overlap, more accurately defined as flightline sidelap, between adjacent flightpaths was too small to accommodate background pixel clipping because gaps would have been created. In fact, the percentage of sidelap was so uniformly small that many between flightpath holidays, or holes, can be seen throughout the completed mosaics. Figure 21 shows flightlines BLINE7E and BLINE8W and their associated mean reflectance spectra without the background pixels. The means and standard deviations have shifted considerably. 


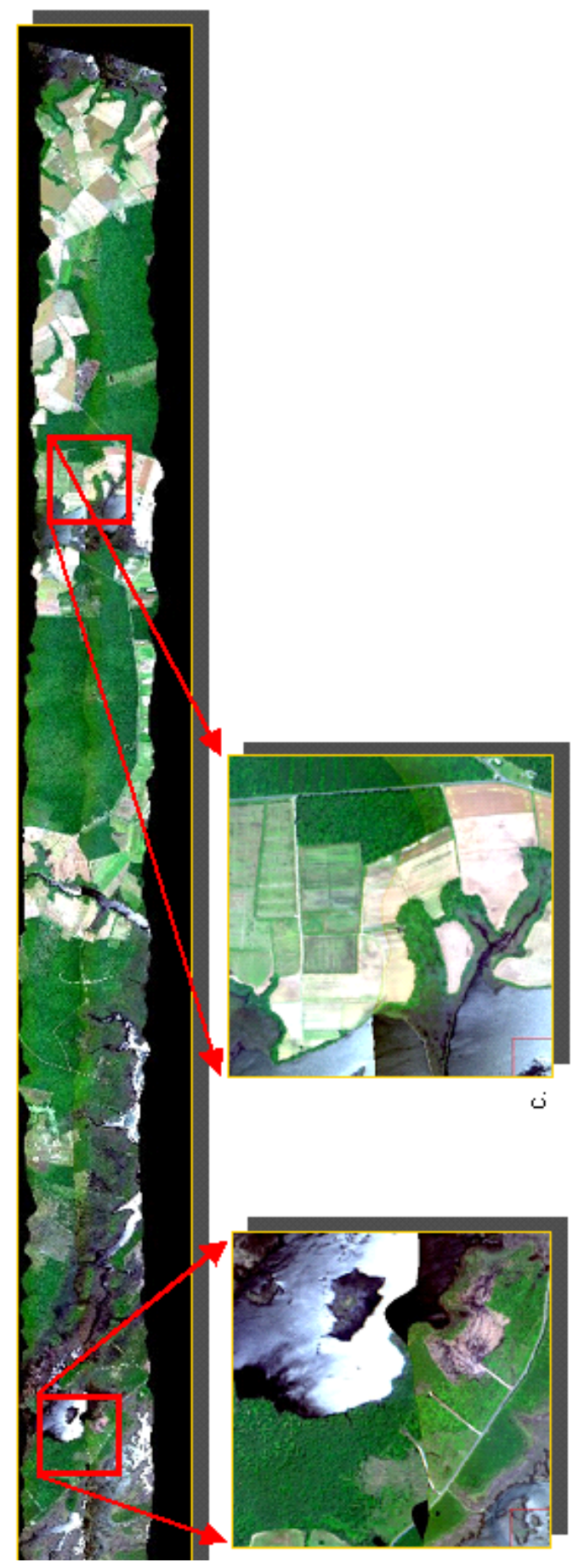

Figure 18. True color mosaic of uncorrected lines BLINE7E and BLINE8W ( $b$ and $c$ provide detail of the overlap regions). 


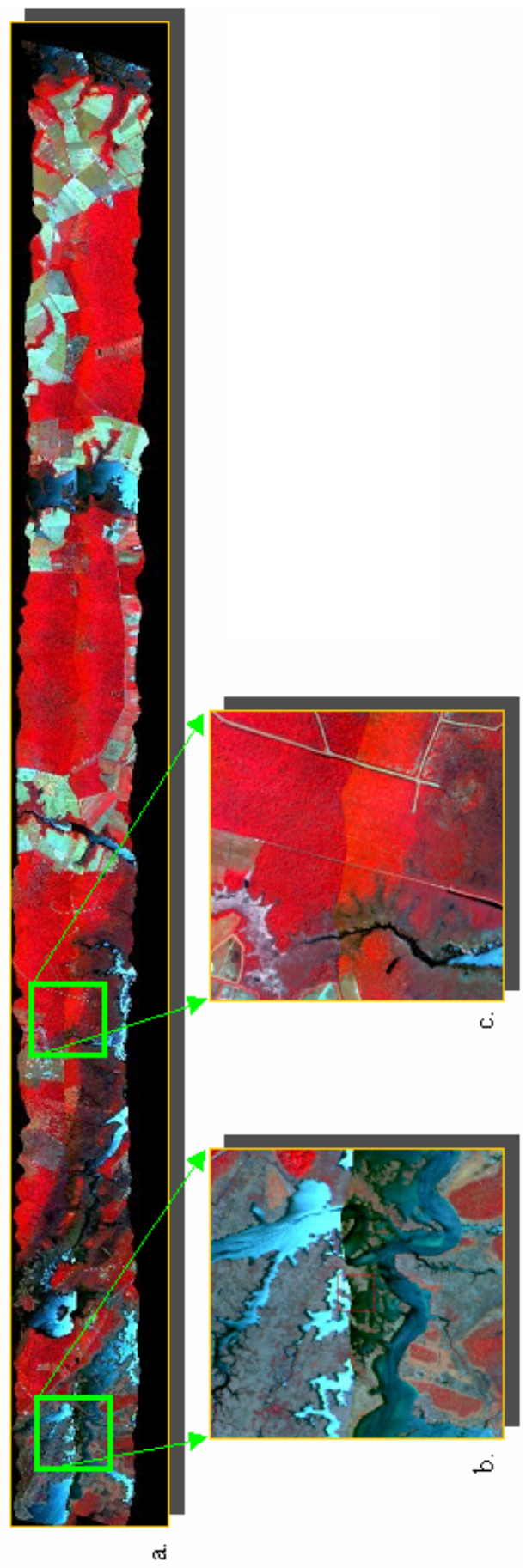

Figure 19. False color mosaic of uncorrected lines BLINE7E and BLINE8W ( $b$ and $c$ provide detail of the overlap regions). 


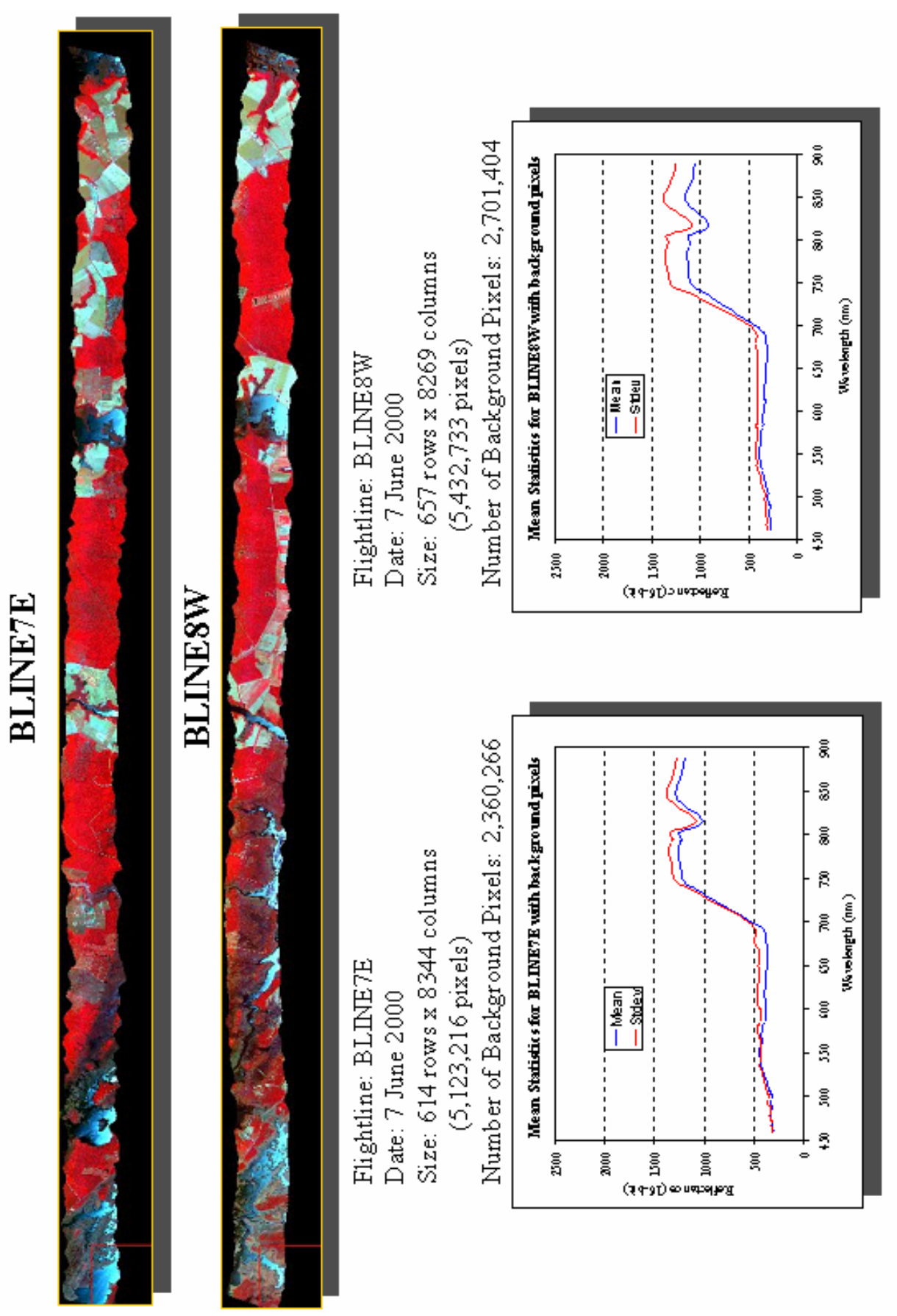

Figure 20. Flightlines BLINE7E and BLINE8W and their associated mean reflectance spectra, including background pixels. 

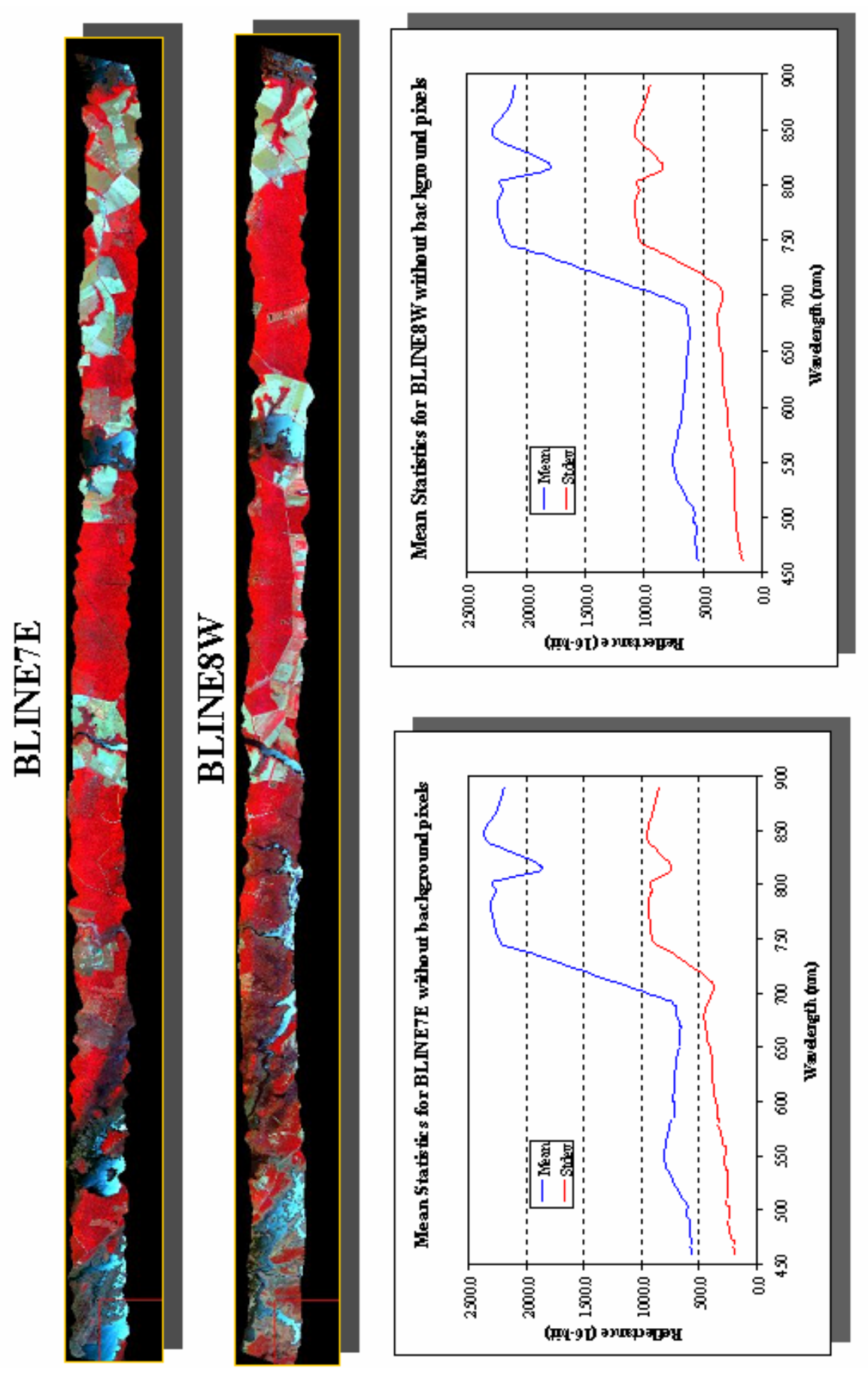

Figure 21. Flightlines BLINE7E and BLINE8W and their associated mean reflectance spectra, without background pixels. 
Reflectance-calibrated imagery is normally plotted using percent as the unit of measure for each pixel. Therefore, pixel values would theoretically have a range of 1 to $100 \%$, assuming that a value of $0 \%$ (or $\mathrm{DN}=0$ ) would be reserved for any background pixels. Commercial image processing software generally does not store and process digital imagery, either reflectance-calibrated and noncalibrated, using DNs scaled from 1 to 100 . The most common digital data formats include:

- Binary data where each pixel has a DN of either 1 or 0 .

- 8-bit integer data with a DN range of $256\left(2^{8}\right)$.

- 16-bit integer data with a DN range of $65,536\left(2^{16}\right)$.

- Floating-point data where DNs are stored as real numbers (positive or negative).

Furthermore, 8-bit and 16-bit data can be stored as signed or unsigned. Signed DNs include a negative or positive sign with each integer value. Unsigned DNs include only positive integers. Calibrated image data should be unsigned; negative DNs should not exist as the theoretical minimum reflectance integer value is +1 . As stated above, the AISA sensor acquires 12-bit data, resulting in a radiometric range of $4095\left(2^{12}\right)$. The value of 0 is reserved for background pixel. Therefore, to conform to image processing software file formats, the calibrated AISA images should be stored in an unsigned (i.e., no negative numbers), 16-bit format with a minimum reflectance DN of 1 and a maximum DN of 4096. While this may not appear to be an acceptable scale for percent reflectance, the 1 to $100 \%$ scale has simply been re-scaled to 1 to 4095 . For example, a reflectance value of $10 \%$ has a 12-bit integer value of 410 (or 409.6 rounded to the nearest whole number). A 70\% reflectance measure is assigned a DN of 2867. Background pixels should maintain a $\mathrm{DN}$ of 0 .

The AISA imagery does not follow these assumptions of the theoretical range associated with 12-bit data. The negative DNs presented a problem. Several flightlines (not shown) had reflectance minima in one or more bands approaching -32,000. A complete assessment of the 38 bands within all flightlines revealed channels 1,2 , and 38 had the most frequent negative DNs. This radiometric problem should be expected from the channels near the extremes of the range of the sensor CCD. That is, at the upper and lower limits of the CCD, the detector likely has the greatest signal-to-noise ratios and the lowest relative output (or responsivity). The number of pixels with negative values ranged from only a few to nearly 10,000. While the larger frequency appears alarming, 10,000 pixels represent less than $0.5 \%$ of the total pixels within each image. Finally, the negative pixels were nearly always assigned to the first and last pixels in the 
cross-track direction. These are the outside pixels along the northern and southern edge of each flightline.

Several methods were employed to deal with the negative DNs. If all the anomalous pixels were indeed located at the edge of the flightline, they were simply assigned a value of 0 , becoming background pixels. In this case, all 38 bands lost these edge pixels to maintain the proper file size of the actual image data. If one or more of the negative pixels within an individual band were located in the interior of the image data, they were renumbered to a value of 1 . This time consuming step in the post-processing the AISA data had very little impact on the overall radiometric quality of the imagery. However, it was thought to be critical in preparing the images for mosaicking. No other manual adjustments to the image histograms were employed.

3.3.2.2 Specular Reflectance or Water Glint. The saturated, or washed-out, water pixels are a normal phenomenon in both digital and analogue images. The AISA images are no exception. The saturation is a result of the specular reflectance properties of water. Specular reflectance occurs when nearly all of the incoming illumination (in this case, sunlight) is reflected from a non-diffuse, or smooth, surface (such as calm water) directly onto the optical focal plane. In other words, the water acts like a mirror, with the reflected sunlight saturating these pixels. Figures 18 and 19 clearly depict these saturated water pixels. Also, note that the glare decreases in intensity from south to north across the image. The glinted pixels diminish towards the north side of the flightline because the mirror (i.e., the water) reflects the sunlight at an angle that passes below the far end of the detector. Any subsequent radiometric manipulations to try to improve the quality of the vegetated pixels would likely be negatively affected by the presence of the glaring water pixels. Therefore, a masking procedure was implemented to remove all the pixels containing standing water.

Several techniques were considered for removing the water pixels. Both supervised and unsupervised classification routines, applied to each flightline independently, were investigated, but were determined to require too much time and employed subjective delineation of land cover classes. Digitizing the water areas was also considered, but again would have required too much effort. The land/water mask for each flightpath was eventually created using the Normalized Difference Vegetation Index (NDVI). This simple mathematical expression uses the ratio of the difference between the reflectance DNs of a near infrared band and a red band as compared to their sum. 


$$
\mathrm{NDVI}=\frac{\mathrm{NIR}-\mathrm{Red}}{\mathrm{NIR}+\mathrm{Red}}=\frac{\operatorname{AISA}(30)-\operatorname{AISA}(22)}{\operatorname{AISA}(30)+\operatorname{AISA}(22)}
$$

where:

$$
\begin{aligned}
& \operatorname{AISA}(30)=\quad \text { band } 30(782 \mathrm{~nm}) \\
& \operatorname{AISA}(22)=\quad \text { band } 22(671 \mathrm{~nm}) .
\end{aligned}
$$

The output floating point formatted image has a histogram range of -1 to +1 . Pixel values below 0 are generally associated with non-vegetated, or only sparsely vegetated, surfaces. Such pixels include man-made features (e.g., roads, roofs), bare earth, and water. As the NDVI values increase, so does the amount of vegetative cover within each pixel to a theoretical maximum value of 1 . Figure 22 shows the resulting NDVI image BLINE7E, as well as the original (i.e., floating point) histogram and the histogram after rescaling to an unsigned, 8-bit radiometric resolution.

To create the land/water mask, the NDVI 8-bit file was segmented into two new images, one with vegetated pixels and one with water pixels. The split was determined by examining the distribution of the NDVI histogram. Ranges of pixel values in the NDVI image associated with the major land cover classes, including water, land, and transition areas, were determined through visual interpretation. Then a precise breakpoint coinciding with the separation of land pixels from water pixels was identified. In the case of BLINE7E, an acceptable break was found at $\mathrm{DN}=145$ (Fig. 22). Finally, the NDVI image was split into two distinct binary masks. The first mask represented water pixels $\left(\mathrm{DN}_{\mathrm{NDVI}}<=145\right)$ and the second represented land pixels $\left(\mathrm{DN}_{\mathrm{NDVI}}>145\right)$. The land mask was applied to the full hyperspectral image such that only a subset of the original file remained depicting only those pixels supporting vegetation. The accuracy of this step was not quantified. However, a visual inspection was employed to determine if water pixels remained in the output "land-only" file. The output image was also inspected to determine if too many land pixels had been removed. If the land-only image was not acceptable, the NDVI histogram was interpreted again and a new breakpoint was selected. Across all flightlines, the breakpoints fell between 135 and 165. Figure 21 shows the vegetated pixels segmented from flightlines BLINE7E and BLINE8W. A plot of the mean DN's for all 38 bands is also provided in Figure 23. All subsequent processing, including image classification, was performed on the vegetation-only images.

3.3.2.3 Reduction in Data Dimensionality. An interpretation of the mean per band reflectance values, shown in Figure 23, suggests problems with data in the near infrared region of the spectrum. Specifically, the pronounced dip in the 
curve at band $34(817 \mathrm{~nm})$ is not normal, indicating a significant radiometric problem. Inspection of the raw (i.e., non-calibrated) radiance image data showed the same anomalous dip in band 34. A more extensive comparison of AISA vegetation spectral signatures to vegetation signatures contained within spectral libraries maintained at TEC revealed that bands 33 through 38 generally did not provide quality reflectance data. This analysis was not quantitative, but was based on extensive experience with analyzing vegetation signatures from more mature hyperspectral imaging systems (e.g., AVIRIS, HYDICE) and from ground-based vegetation signatures collected using hand-held spectroradiometers. The decision was made to drop these bands from the images. Evaluation of the remaining 32 bands suggested that many could likely be dropped without any significant impact to image classification accuracy. Again, this was a qualitative evaluation based on research team experience. Therefore, of the original 38 bands, a total of 14 were retained.

As stated above, signal-to-noise ratios for bands 1 and 38 severely diminished the quality of these bands. However, band 1 is the only channel close to the center of the blue region of the spectrum $(\sim 450 \mathrm{~nm})$ and was kept. Similarly, band 38 , while of relatively poor quality, was kept to at least provide a signature end point well into the near infrared region. Figure 23 depicts a plot of the average DNs for BLINE7E and BLINE8W after this reduction in data dimensionality.

An additional benefit of reducing the dimensionality of the data was a significant decrease in the file size. For example, the full 38 band flightline BLINE7E is $\sim 380 \mathrm{MB}$. After removal of 24 bands the file size is reduced to $\sim 140$ MB. By working with smaller files, subsequent image processing steps required much less CPU time.

3.3.2.4 Cross-Track Illumination Correction. Test mosaics created using the 14-band, vegetation-only images still had pronounced seams where adjacent flightlines overlapped. The mosaics showed that the south edge of each flightline was consistently darker than the north edge of the overlapping flightline within forested areas. An inverse shift in brighter to darker pixels was observed in the water and marsh vegetation pixels, with brighter pixels at the lower edge of the flightlines. This significant departure in the supposedly calibrated data was attributed to cross-track illumination irregularities, which can be caused by any number of factors, including:

- Within sensor (i.e., system) radiometric distortions.

- Flawed reflectance calibration.

- Bi-directional reflectance influences. 


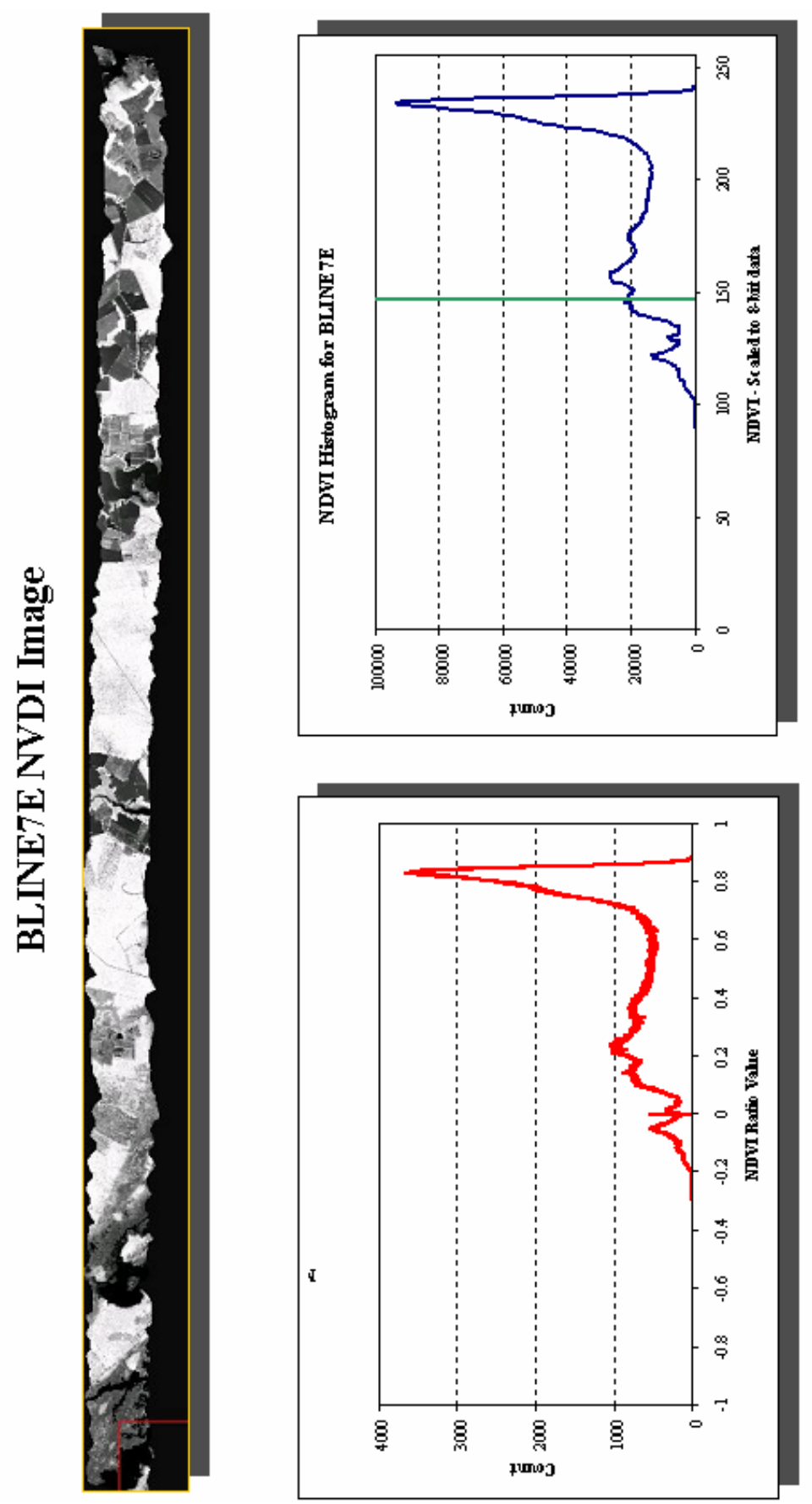

Figure 22. Example of NDVI image. Graphs show floating point NDVI values and NDVI values scaling to 8bit data. 

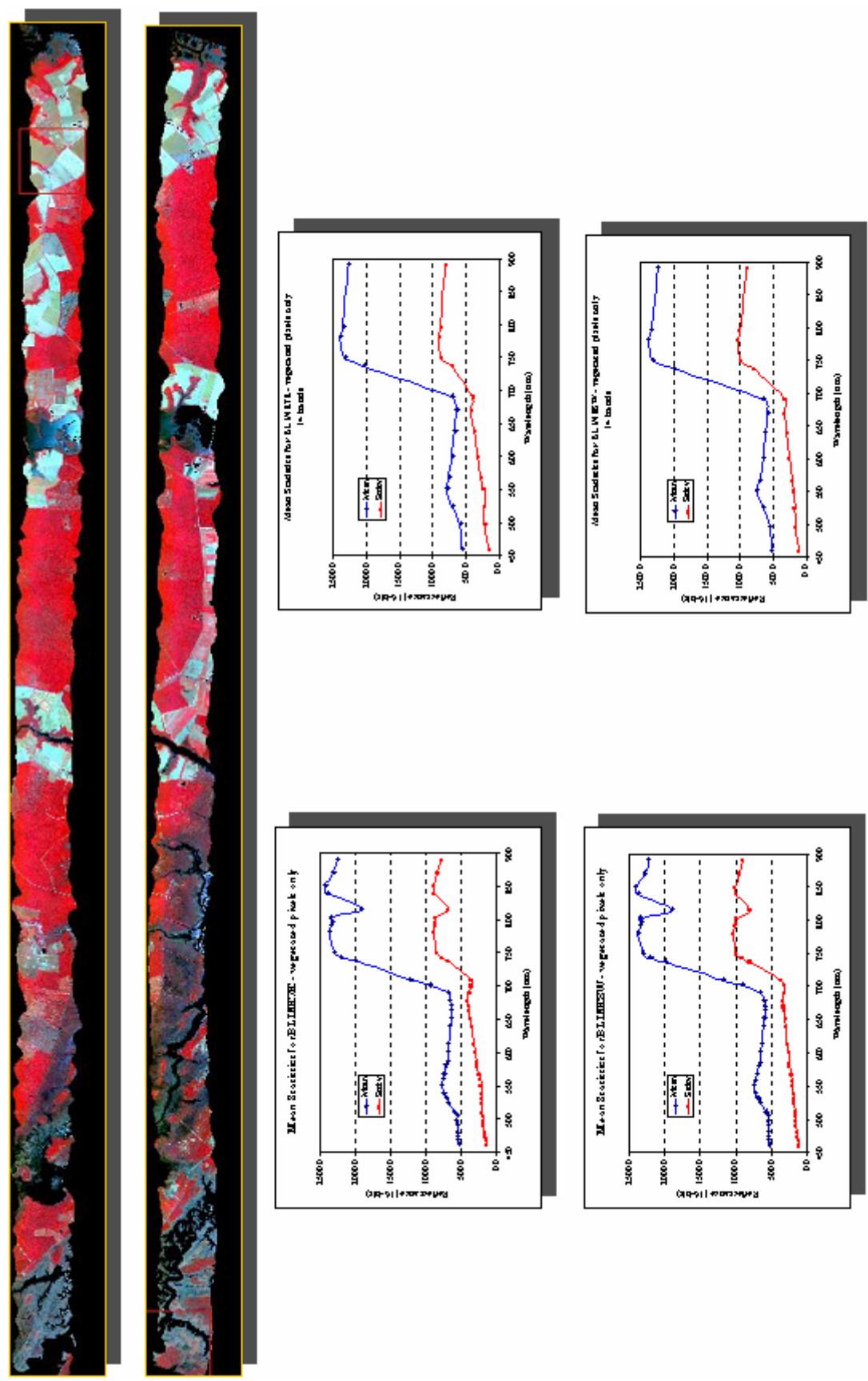

Figure 23. Flightline BLINE7E and BLINE8W and their associated mean reflectance spectra after removal of water pixels and reduction in data dimensionality. 
Regardless of the cause, the illumination anomaly within all of the images represented a significant problem in creating usable mosaics. The ENVI imaging processing software offers a cross-track illumination correction. The routine calculates the overall row (east-to-west) average DNs within an image and then fits a polynomial to the averages, for each band, to potentially correct (or flatten) the reflectance data in the cross-track direction (south-to-north). If applied properly, the darker southern edge of each image should brighten, while the pixels at the northern edge should darken slightly. This correction routine was applied to each flightline after removal of the water pixels. The correction algorithm ignores the background pixels (i.e., $\mathrm{DN}=0$ ) when calculating the across-track averages and when applying the polynomial functions.

3.3.2.5 Histogram Equalization. As a final step in preparing adjacent images for mosaicking, an attempt was made to equalize the histograms of two overlapping flightlines. This technique uses a simple mathematical formula to match the general shape of the mean DN curves between two image files. Figure 24 displays the mean and standard deviation DN curves for the 14-band images BLINE7E and BLINE8W. These curves were calculated using only the overlap regions of the two adjacent flightlines. The formula employed to match one histogram to the other is:

$$
\text { DNout }=\left[\left(\frac{D N i n-\mu D N t}{\sigma D N t}\right) \times \sigma D N r\right]+\mu D N r
$$

Where

$$
\begin{aligned}
\text { Dnout } & =\text { output pixel value } \\
D N i n & =\text { input pixel value } \\
\mu D N t & =\text { mean pixel value of transform image } \\
\sigma D N t & =\text { standard deviation of transform image } \\
\sigma D N r & =\text { standard deviation of reference image } \\
\mu D N r & =\text { mean pixel value of reference image. }
\end{aligned}
$$

Flightline BLINE7E was transformed to the reference image BLINE8W. Within the image processing software, the equation is applied to the 14-band input image (BLINE7E) using matrix algebra. The four mean and standard deviation terms are one-dimensional arrays (or vectors). The output image is an adjusted 14-band image. Figure 25 depicts the output mosaic, as both a true-color and false-color file. While the equalization calculation worked nearly perfectly, 
as is displayed by the second mean/standard deviation graph, the correction still left a noticeable seam between adjacent flightlines.
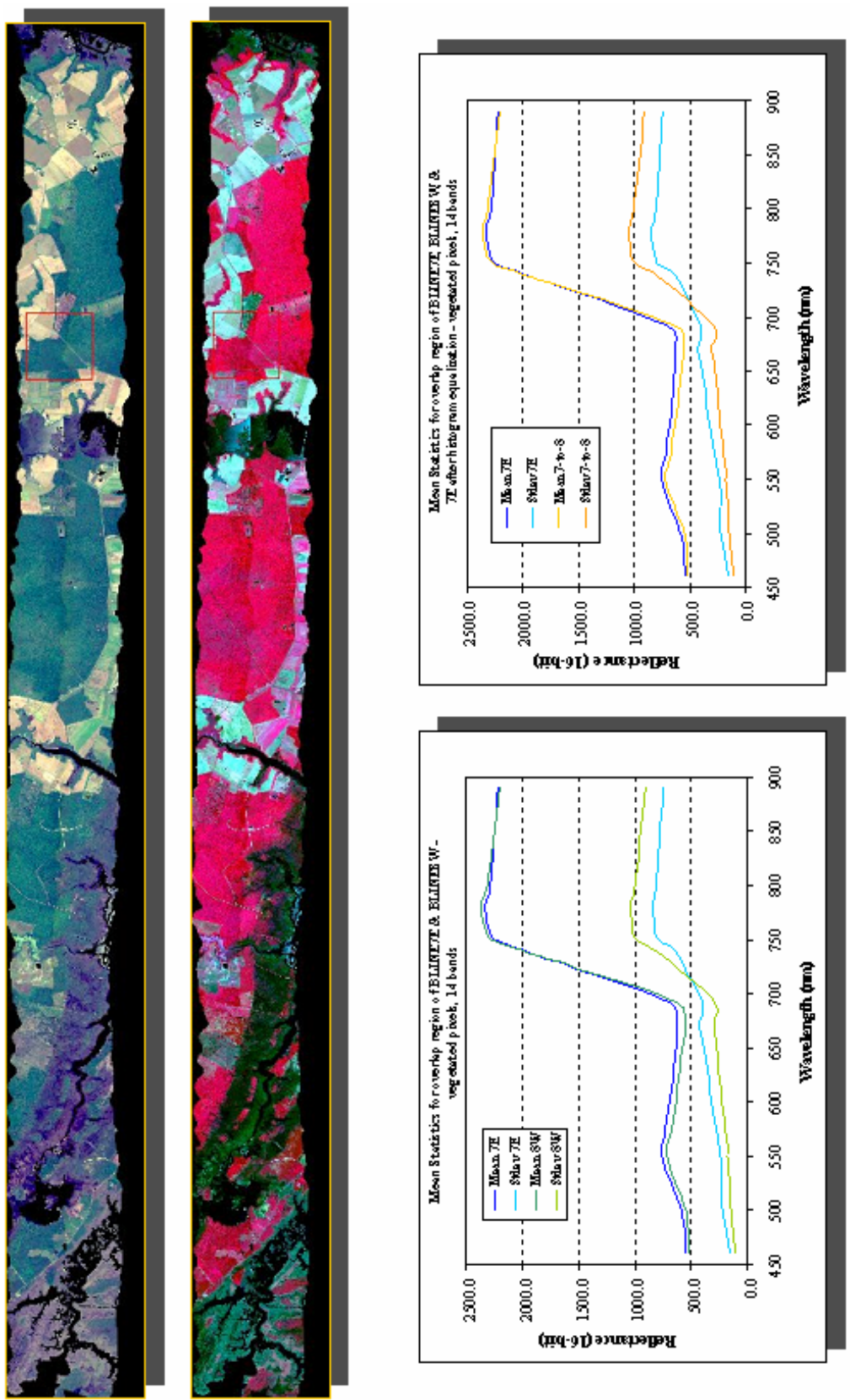

Figure 24. Histogram equalization results between BLINE7E and BLINE8W. 
a

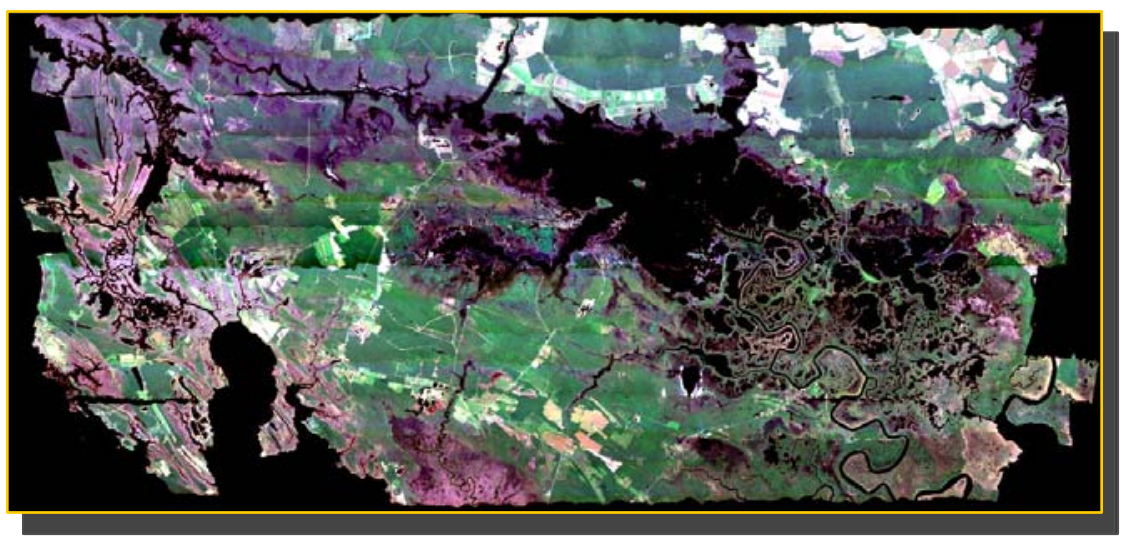

b.

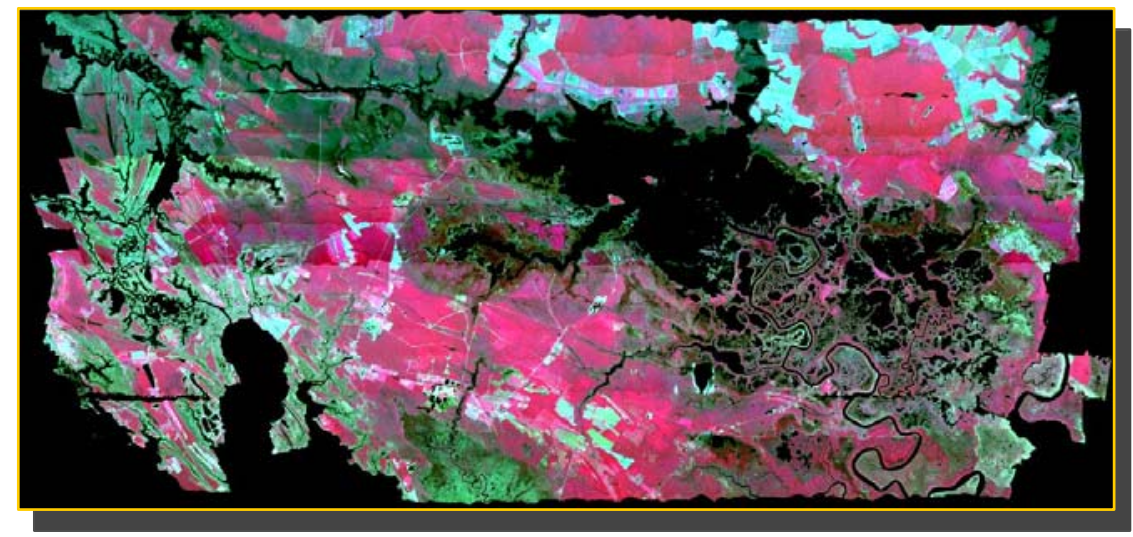

Figure 25. Southern mosaic-flightlines BLINE7E through BLINE20E (a-true-color composite; b-false-color composite).

The technique was more successful between some pairs of flightlines and less successful between other pairs. Figure 25 displays the final output mosaic of flightlines BLINE7E through BLINE20E. Several seams are clearly visible. The most prominent is the seam between flightline 10E and 11E. Repeated attempts to improve the significant radiometric distortion between these two flightlines were equally unsuccessful.

\subsubsection{Mosaicking}

At the onset of this project, it was assumed that this would be the only postprocessing step that would be required to prepare the AISA images for vegetation classification. However, an array of additional post-processing techniques was applied to each AISA flightline to try to improve radiometric anomalies and distortions. The quality of the resulting mosaics, in terms of consistent reflectance values between and among the individual images, remained marginal. 


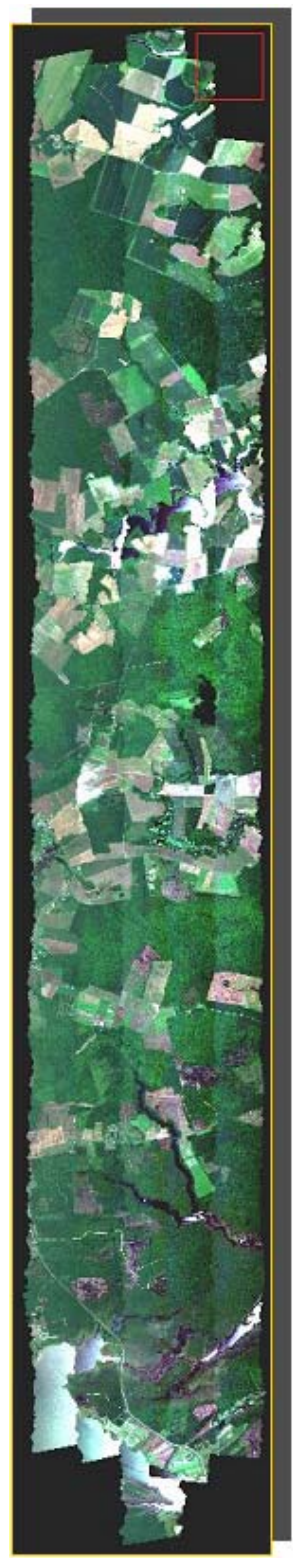

๘่

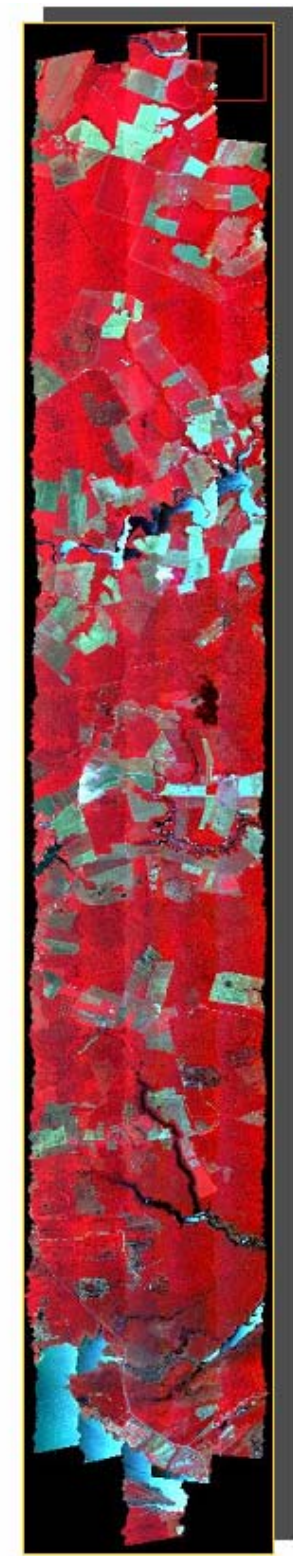

$\dot{0}$

Figure 26. Final mosaics for flightlines 1 through 5 acquired on 30 June 2000 (a is a true color composite and $b$ is a false color composite).

Flightlines BW1E1, BW2E, BW3E, BW4W, and BW5W, all acquired on 30 June 2000, produced the highest quality mosaic (Fig. 26). During repeated at- 
tempts to remove seams between overlapping flightlines and to equalize the reflectance values, the best result for the final mosaic included the water pixels. The relatively small percentage of water pixels within these flightlines meant that the specular reflectance (i.e., glint or glare) problem had little effect on the quality of this mosaic. Seams are still visible and the histogram equalization procedure was moderately successful. 


\section{FIELD DATA COLLECTION}

Ground-truth data collection, or site characterization information acquired for each reference wetland in the Poplar Island Restoration Project, was not included as part of this pilot project to minimize cost. Likewise, the levee assessment project's goal was to see if remotely sensed data could take the place of ground inspections.

\subsection{Invasive Species Mapping at Lake Okeechobee, Florida}

Field data were acquired during acquisition of both the CAMIS missions. Additional field data were not collected at the time of IKONOS acquisition. Plant species associations were recorded at point locations throughout the three study areas. Differentially corrected GPS coordinates were recorded at each sample plot. Terrestrial photos were also taken to qualitatively document the density and distribution of the emergent and submerged plant species. During the January 2001 mission, a total of 22 sites were sampled. During the December 2001 mission, a total of 36 sites were sampled (Fig. 27). Considering the Corps' primary mission is to manage water hyacinth and water lettuce, areas supporting these two species were more intensively sampled. None of the January 2001 field data were applicable to the December 2001 imagery owing to significant changes in vegetation distributions between the dates.

\subsection{Wetland Vegetation Mapping Over Blackwater Wildlife Refuge}

Four brief field data collections, in support of this image classification effort, were completed during the Fall of 2001 and Summer of 2002. USFWS staff at the Refuge provided the 2001 data, while TEC staff collected a small set of additional field sites in 2002. Plot coordinates, collected using Garmin hand-held GPS receivers, are in the UTM projection with datum WGS84. These coordinates were not differentially corrected. Therefore, the average coordinate accuracy is assumed to be between 5 and $10 \mathrm{~m}$. As shown in Figure 28, sample sites WP001 through WP023 fell within the 30 June mosaic. Sites WP024, WP025, and WP026, as well as the 7 August 2002 sample sites, were located in the 7 June mosaic, adjacent to Shorters Warf Road. 


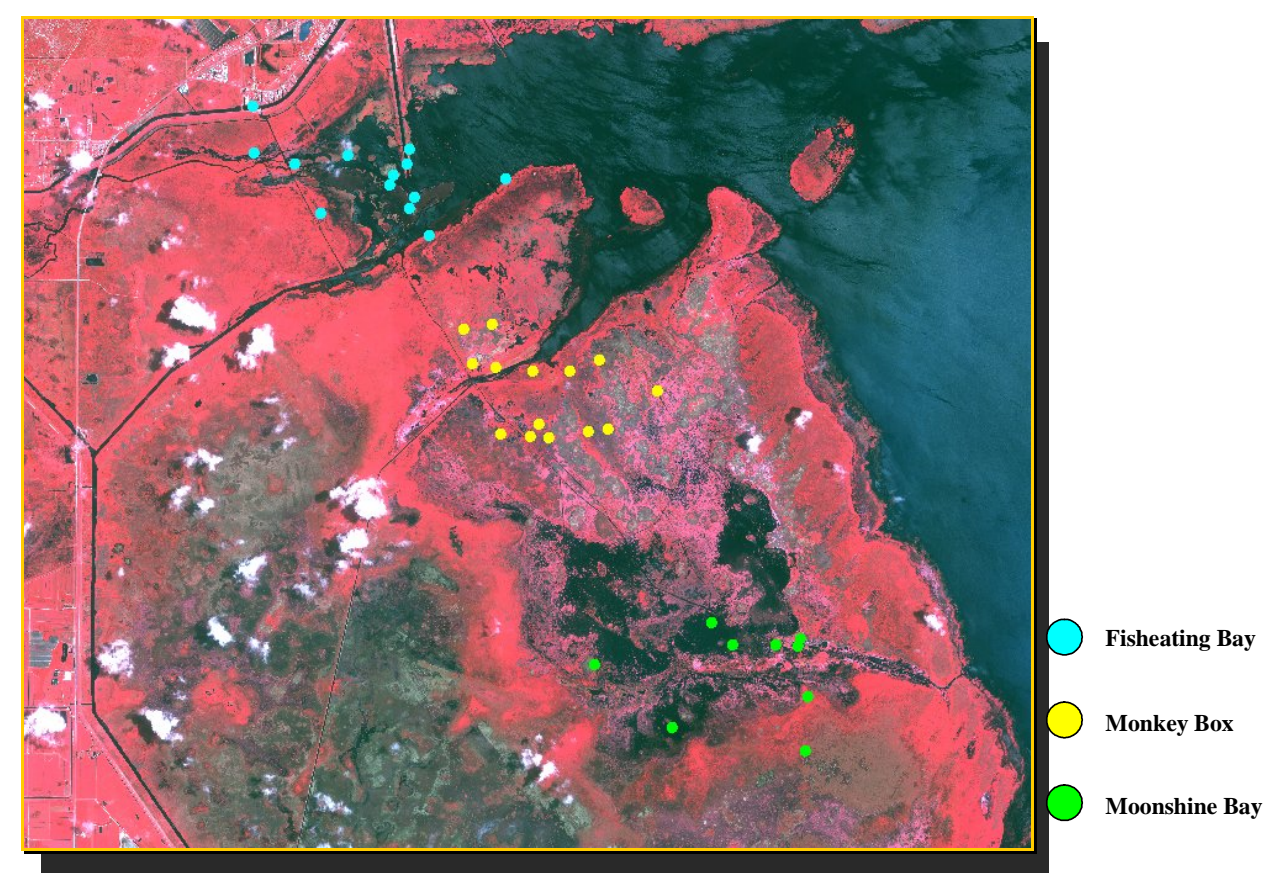

Figure 27. Location of field plots established during 9 December 2001 CAMIS image acquisition.

The Refuge provided additional field data, collected during the summer of 2000. These data were reported as percent vegetative cover, by species, within 1$\mathrm{m}^{2}$ quadrats. The plots were divided into five areas (2 through 6 ) throughout the southeast portion of the Blackwater Refuge and the northeast portion of the Fishing Bay Wildlife Management Area. Areas 2 and 3 were located just west of Shorters Warf Road. Areas 4, 5, and 6 were to the east, in the Fishing Bay Area, and were not located within the southern ASAI mosaic. The 240 quadrats within areas 2 and 3 supported non-woody vegetation. Litter, mud, or water occupied non-vegetated areas within the plots. Only those plots with at least $50 \%$ cover by any of three dominant species were used for spectral signature extraction. Given that the radiometric variation between some flightlines in the southern mosaic approached $20 \%$, it was assumed that subtle spectral variations associated with mixed species compositions in many plots would be lost in the between-flightline noise. Therefore, a total of 111 quadrats were determined to be suitable for signature extraction. Figure 29 displays the locations of these sample sites and indicates the dominant species present. The accuracy of the geographic coordinates for these data is unknown. 


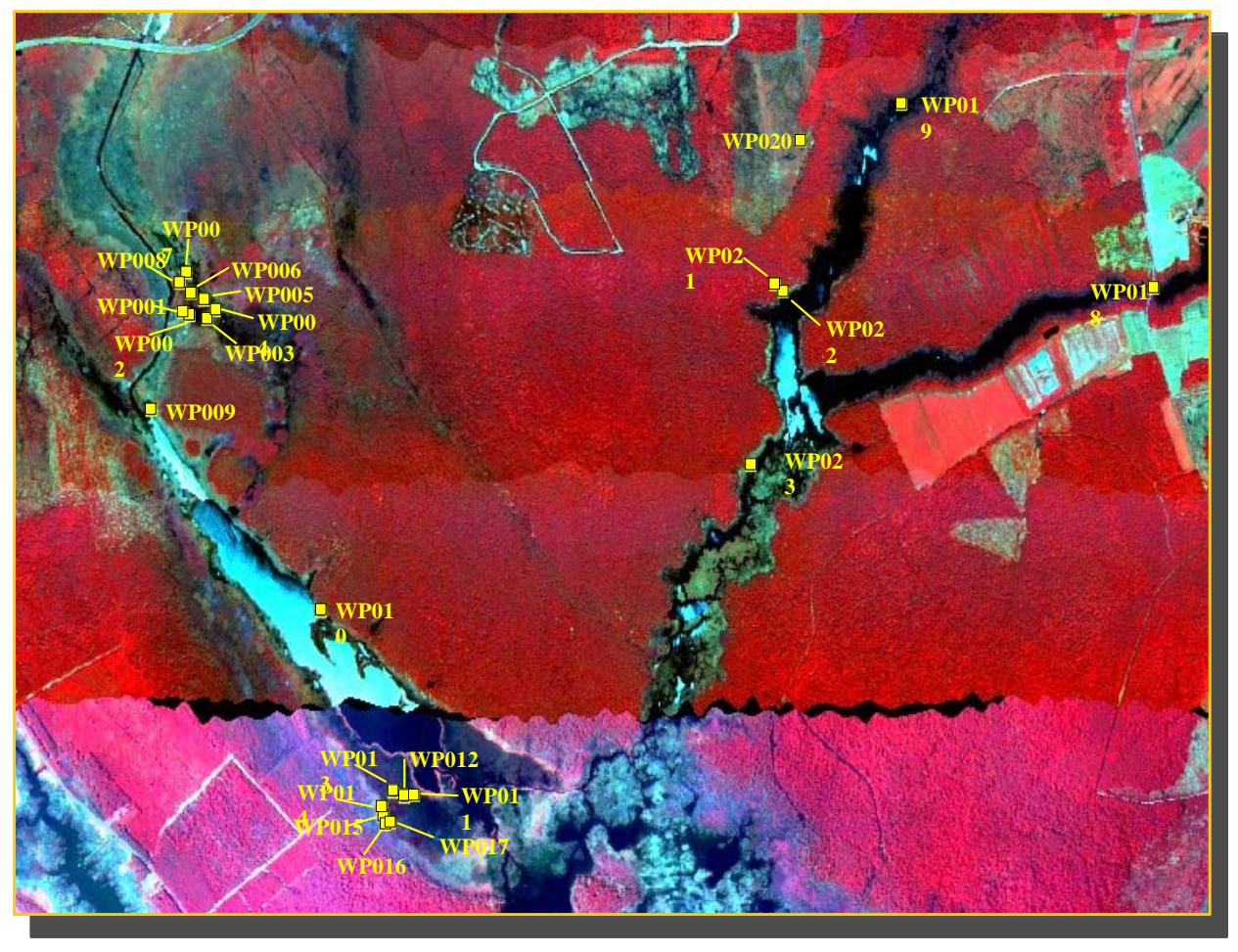

Figure 28. Location of training sites within western part of northern mosaic.

The Refuge also provided a forest type map, in the form of an ArcView shapefile (Fig. 30). The database was exported and deciphered, with a total of seven forest cover types identified, including cutover stands, loblolly pine dominated, loblolly pine-oak mixed, loblolly pine-hardwood mixed, mixed hardwood, morbid/declining stands, and stands with scattered overstories. The shapefile polygons were displayed as overlays on the mosaics and suitable locations within individual stands were selected for training data extraction. A total of 28 training sites were delineated. 


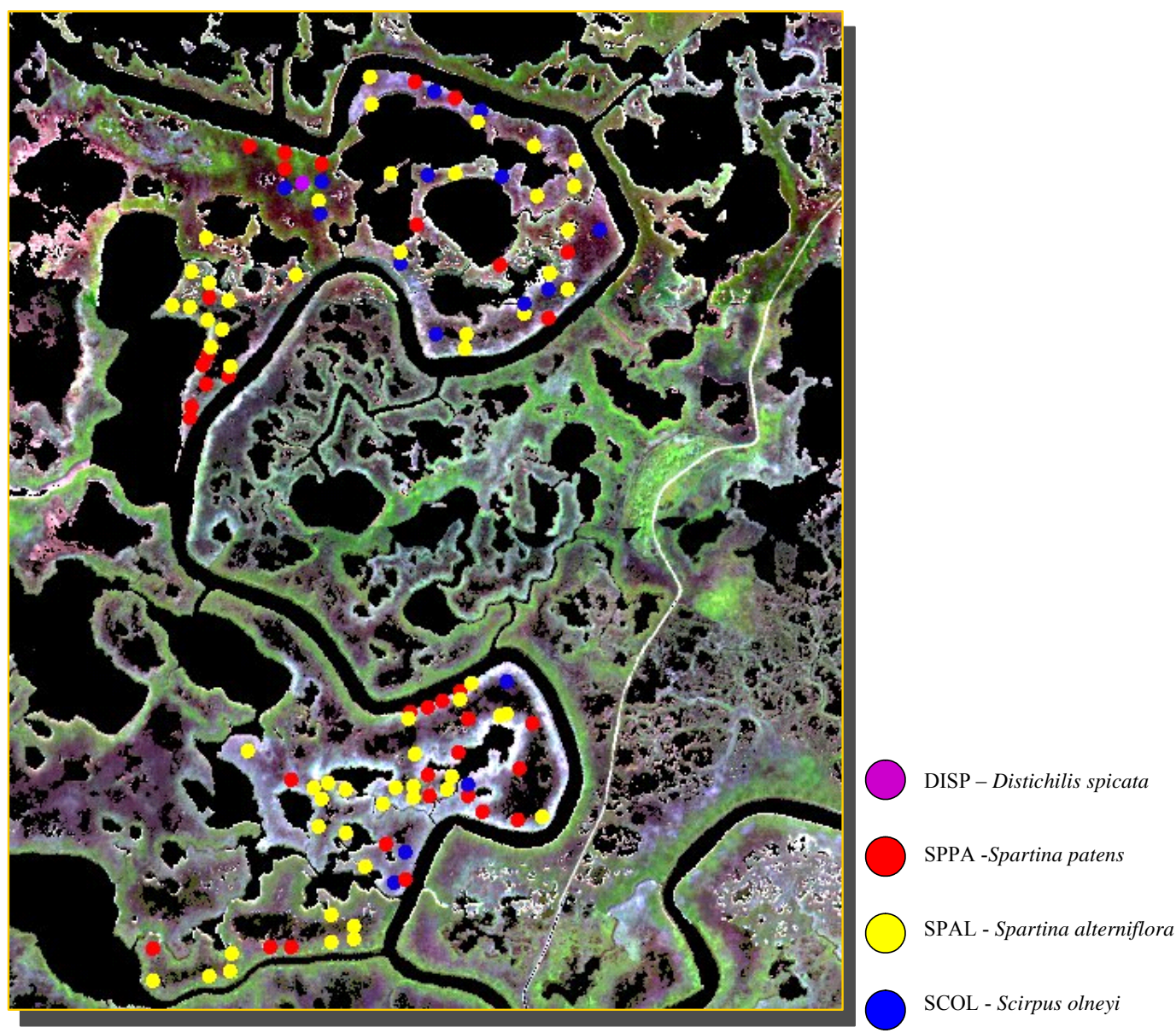

Figure 29. Location of training sites adjacent to Shorters Warf Road. 


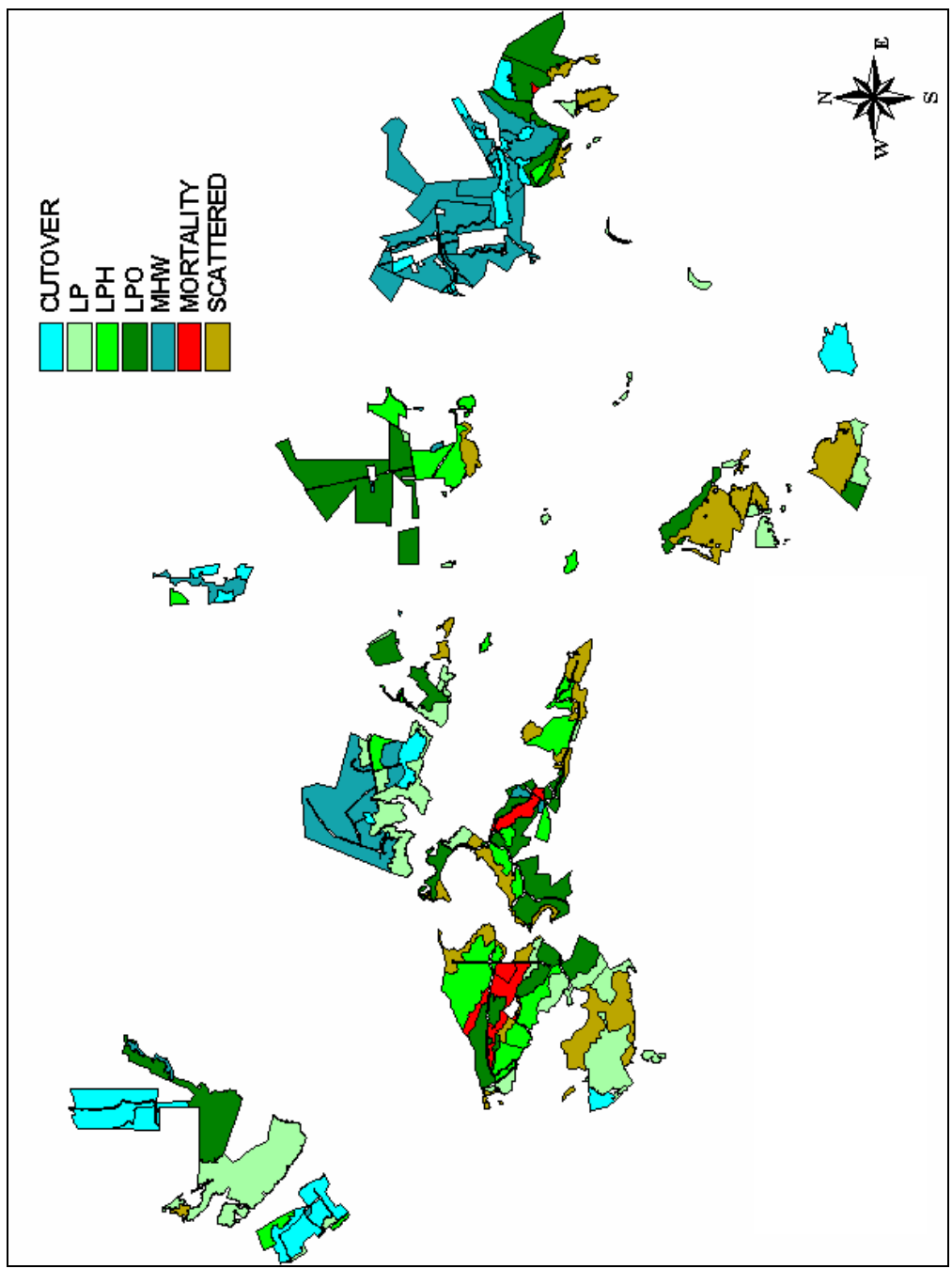

Figure 30. ArcView shapefile polygons used to collect training spectra for classification of forest types. 


\section{IMAGE CLASSIFICATION}

Classification of remotely sensed imagery, using either manual or automated techniques, typically requires ground-truth information and accuracy assessment. Ground-truth data quantifies vegetative parameters, such as species composition and canopy densities, within discrete, geo-referenced sample plots throughout the study area. These sample data statistically train the classification of the entire data set. For image processing applications, ground-truth sample plots are precisely located within the imagery. Multivariate statistics (i.e., training statistics) that define the unique spectral responses of the vegetative classes are extracted from the imagery at these locations. Supervised classification algorithms, a subset of a larger group of statistical techniques called discriminant analyses, use the training data to assign each pixel to one of many discrete vegetation classes.

If training data are absent, more interpretive discriminant analysis techniques are available to classify remotely sensed imagery into discrete land cover classes. Unsupervised classification, or nonhierarchical clustering, is a standard imageprocessing tool used to delineate spectrally unique feature classes, or clusters. This technique is more subjective than the supervised technique, relying on image analyst interpretive skill to accurately classify each spectrally unique cluster.

\subsection{Poplar Island Unsupervised Classification}

As the specific objective of the Poplar Island effort was to delineate only three broad land cover classes without ground truth, a modified unsupervised classification technique was employed. The modified approach included:

- Segmenting each mosaic into two primary classes (vegetation vs. nonvegetation) using the Normalized Difference Vegetation Index (NDVI).

- Classifying each primary segment into eight land cover types using an unsupervised clustering routine.

- Combining the two cluster maps into a final classification.

The resulting class maps displayed 16 land cover types, ranging from deep water (typically the darkest feature), to exposed tidal mud flats, to marsh vegetation of varying density and species composition, to evergreen forests, to sand (typically the brightest feature).

No attempt was made to collapse the 16 classes into the three land cover types required for the Poplar Island restoration effort. Because of the absence of site-specific ground truth data, the 16 classes could be combined (i.e., recoded) in various combinations to produce a final class map depicting water, intertidal 
zones, and vegetation. Therefore, the end user has the flexibility to assign each of the 16 interpreted classes into one of the three general land cover types. Of particular concern are the transition classes that will define the boundaries between 1) persistent water and intertidal zones, and 2) sparsely vegetated intertidal areas and non-tidal vegetated marshland. Field personnel familiar with each site will likely be needed in the end to accurately determine which groups of the 16 classes to collapse to produce three classes. Note that each of the individual mosaics was classified independently.

The class names were assigned based on the spectral response (i.e., cluster spectral signature statistics) of each cluster relative to its statistically adjacent clusters. This was a subjective process. Therefore, the end user must ultimately assign the most accurate cluster names during the recoding of the 16 classes to the three primary land cover types.

\subsubsection{NDVI Mask for Poplar Island Restoration}

Figure 31 shows an example of the NDVI image for the Knapps Narrows site. The NDVI value for each pixel is calculated using the following equation, which is the same equation used earlier on AISA data:

$$
\mathrm{NDVI}=\frac{\mathrm{CB}(4)-\mathrm{CB}(3)}{\mathrm{CB}(4)+\mathrm{CB}(3)}
$$

where

$$
\begin{aligned}
& \mathrm{CB}(4)=\text { the infrared CAMIS band }(800 \mathrm{~nm}) \\
& \mathrm{CB}(3)=\text { the red CAMIS band }(650 \mathrm{~nm}) .
\end{aligned}
$$

The NDVI operation outputs a single raster layer. Therefore, the pixel values are displayed using a grayscale (black and white) lookup table. The unique value assigned to each pixel ranges from -1 to +1 . Values approaching -1 indicate unhealthy or dead vegetation. As live vegetative biomass increases, the index value also increases to a maximum of +1 . Figure 29 also shows the resulting histogram of pixel values derived from the NDVI image for Knapps Narrows. The NDVI image for each reference wetland was split into two segments by visually interpreting the NDVI histogram, the NDVI image, and the original multispectral mosaic. The first image segment contained all non-vegetation areas, depicted with darker pixels; the second contained the brighter vegetated pixels. This subjective distinction was not completely accurate, as pixels near the center of the NDVI histogram (i.e., near the threshold value) represented mixed pixels, or pixels displaying spectral properties of both vegetated and non-vegetated features. Leaf-off 
conditions during image acquisition meant that the deciduous trees and shrubs were without leaves. In addition, the reed and grass species were fully senesced. The lack of photosynthetically active vegetation may have somewhat diminished the overall interpretability of the NDVI image by reducing the contrast between vegetated and non-vegetated segments. However, the NDVI image still was able to adequately separate those pixels that were primarily vegetation from those pixels that did not include vegetation. This is based on near infrared reflectance properties of chlorotic and necrotic vegetation being still very different from the near infrared spectral response of non-vegetated features, such as water and bare soil.

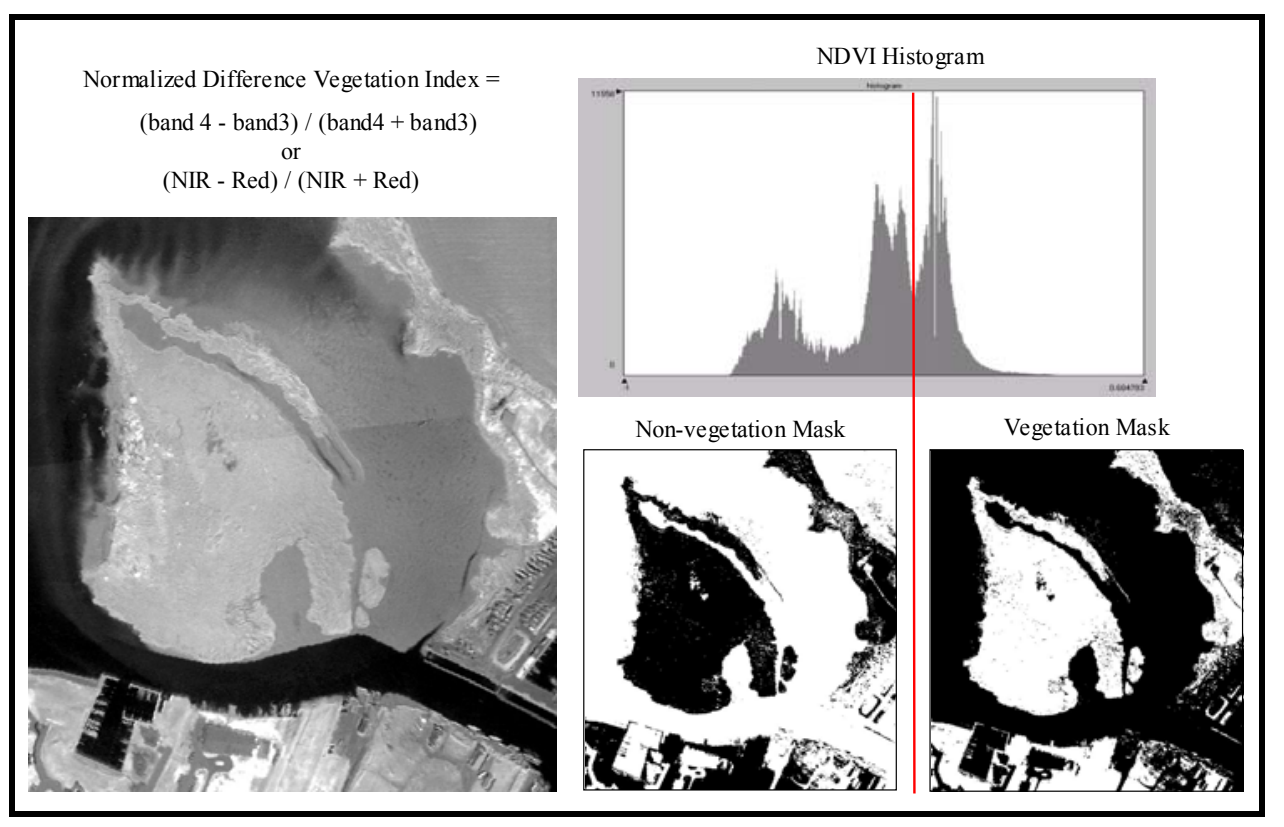

Figure 31. NDVI image (left), NDVI histogram (upper right), and primary NVDI masks.

The segmented images for each mosaic were converted into two masks. An image mask assigns a value of 1 to pixels that are "switched on" and a value of 0 to pixels that are "switched off" (Fig. 31). The non-vegetation mask had all nonvegetation pixels assigned a value of 1 , and all vegetation pixels assigned a value of 0 . The vegetation mask maintained the reverse values (i.e., veg $=0$, non-veg $=$ 1). Each mask was independently applied to the original four-band mosaic creating two unique multispectral images (Fig. 32). One image contained primarily vegetated pixels and the other contained the remaining non-vegetated pixels. 


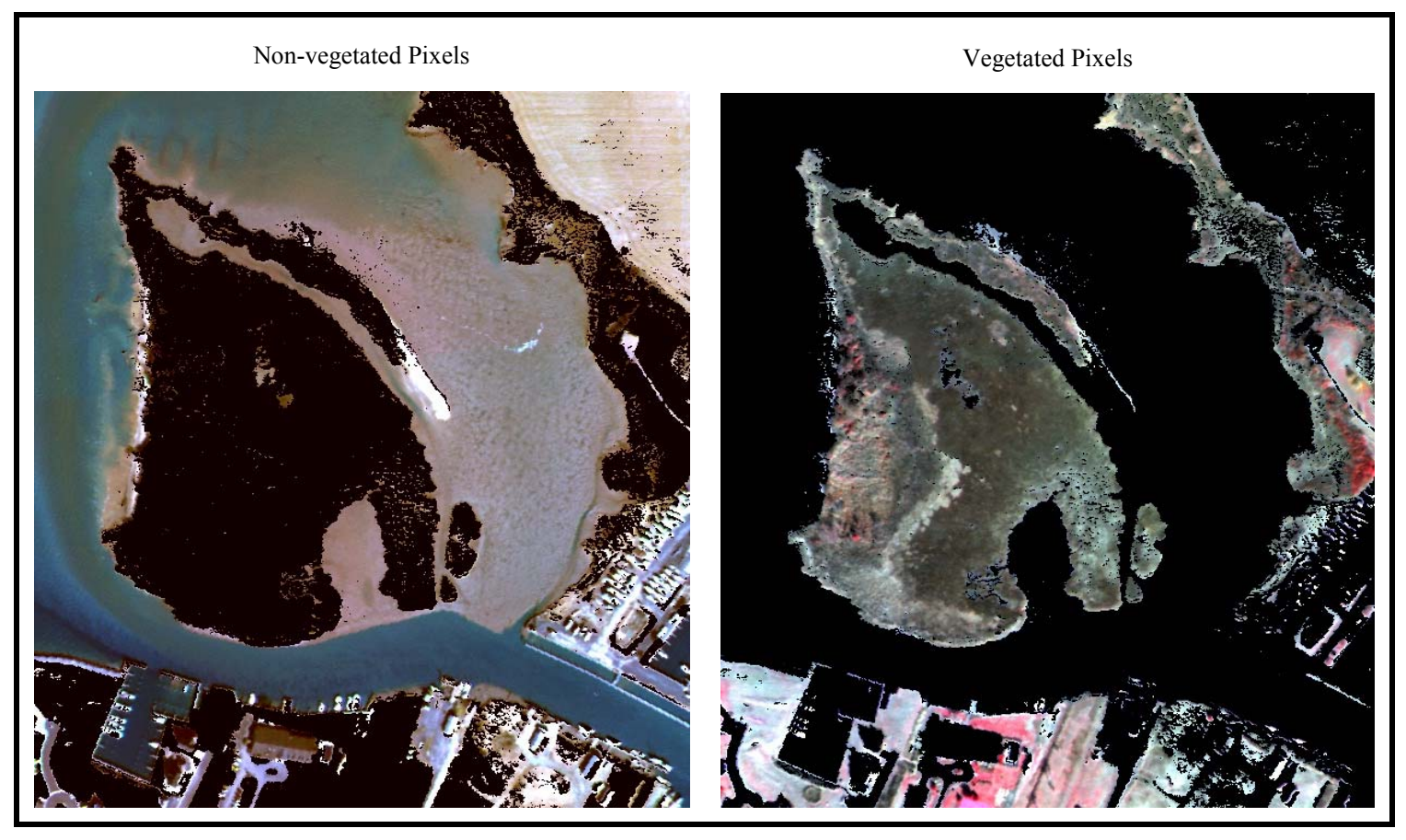

Figure 32. Primary image segments after NDVI masking.

\subsubsection{Unsupervised Clustering}

ERDAS Imagine image processing software contains a standard nonhierarchical clustering routine. This unsupervised classification algorithm was applied to both the non-vegetated and the vegetated four-band, multispectral image segments created by the NDVI masking procedure. Imagine allows the analyst to determine the total number of land cover classes (or clusters) classified within the input image. For this study, eight thematic classes were delineated within each of the primary segments (vegetated and non-vegetated). The selection of eight classes for each of the two image segments was based on field experience at sites similar to the reference wetlands. A subjective evaluation of the general land cover structure and relative abundances of the vegetated and non-vegetated surface features suggested that eight clusters would sufficiently classify each segment. Using the original four-band mosaic as an interpretative replacement for ground-truth data, the analyst assigned a land cover designation to each of the eight unique spectral classes was (Fig. 33). Finally, combining the segmented thematic images produced a complete land cover classification of the area of interest (Fig. 34). 
Land cover designations needed to be modified during compilation of the final class map for each site. Many of the class names are relatively imprecise or primitive in describing the land cover features. In addition, many classes are associated with two or more cover types, with some including both vegetated and non-vegetated features.

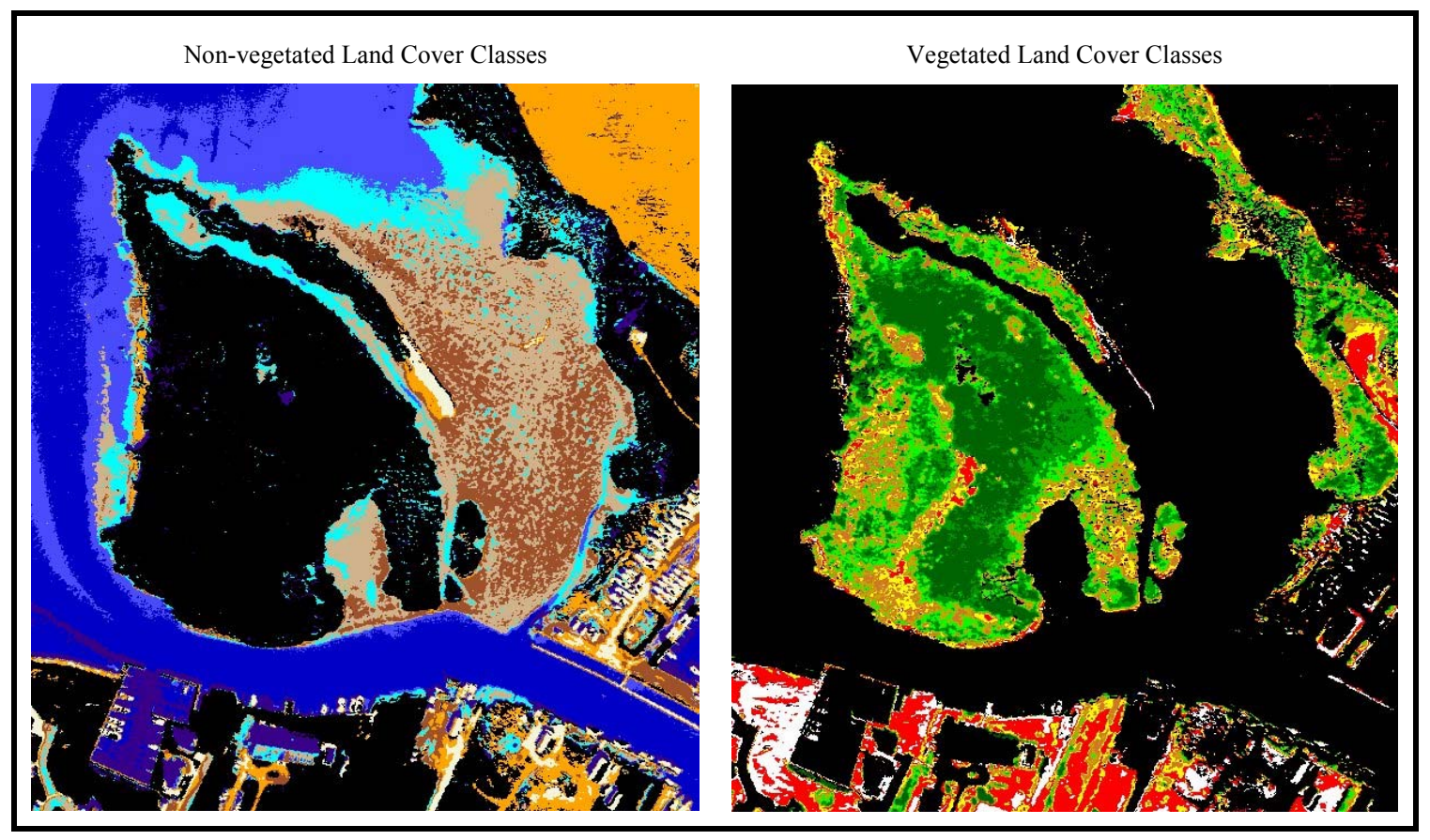

Figure 33. Results of unsupervised classification of each primary segment. 


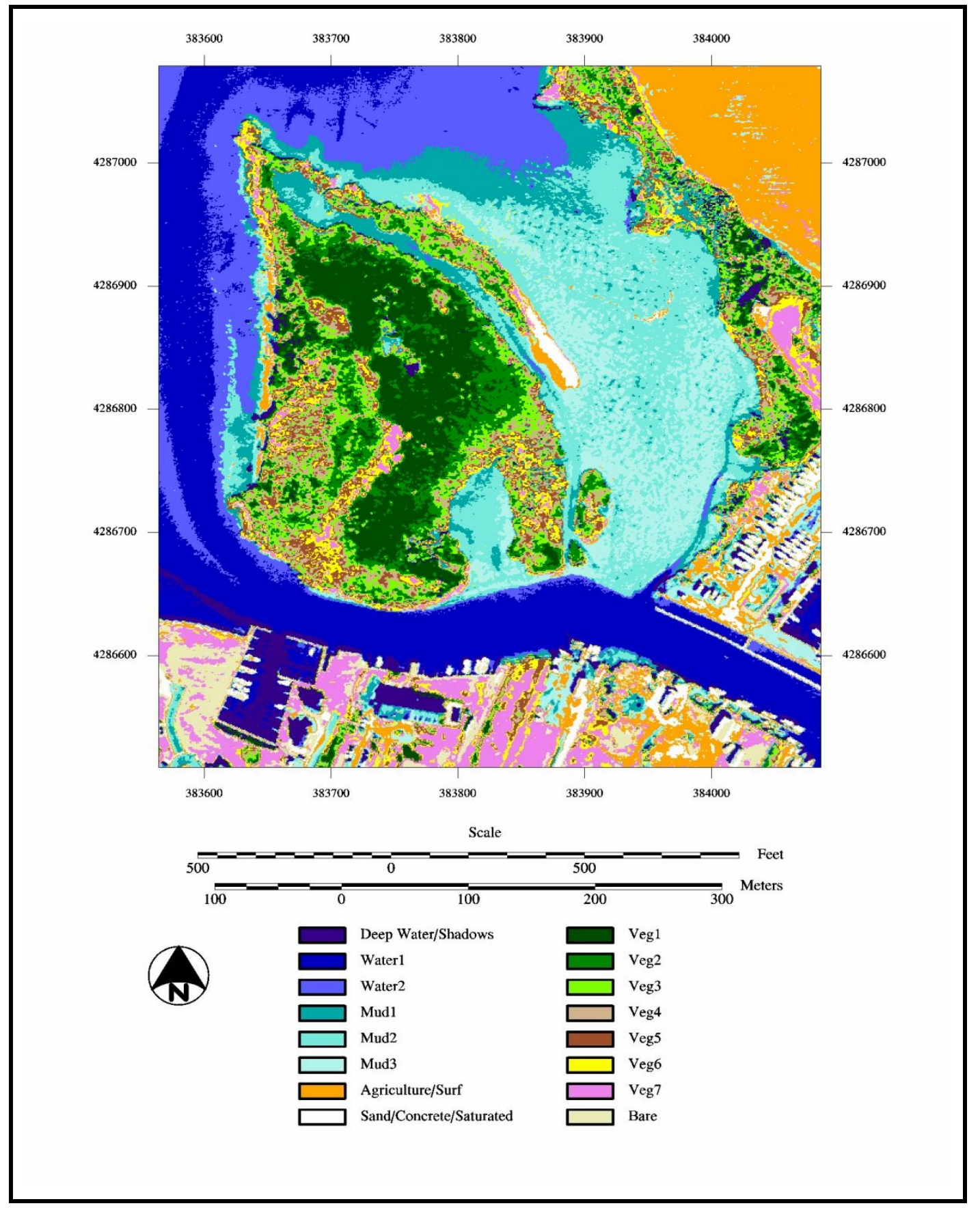

Figure 34. Class map of Knapps Narrows-full resolution. 


\subsubsection{Minimum Mapping Unit}

Very high-resolution imagery typically provides far more detail than can be efficiently used for landscape mapping. For example, high quality, large-scale aerial photography will provide adequate clarity and detail to accurately identify surface features that are no larger than a few square feet. It is unlikely that a photo interpreter would be required to delineate image features only a few feet in diameter. Instead, the analyst will work within the limits of a predefined Minimum Mapping Unit (MMU). This two-dimensional area represents the minimum size of the polygon delineated within the aerial photo. Land use/land cover classifications commonly use a MMU of 1 acre to 1 ha for large scale maps and much larger MMU sizes, such as 10 to 100 ha for very small-scale land cover maps. The MMU used in this application is very small as compared to most land cover classification schemes. All reference wetland class maps were subjected to a nine-pixel $\left(\sim 6.8 \mathrm{~m}^{2}\right)$ minimum mapping unit filtering routine. This two-step routine first uses a simple raster GIS technique to find and remove all raster polygons that are less than or equal to nine pixels. A raster polygon is a contiguous group of pixels that have the same land cover class designation. The thematic image is then "sieved" to remove those raster polygons below the nine-pixel minimum threshold.

The "holes" (removed raster polygons) are then filled by iteratively passing a $3 \times 3$ majority filter over the image until all of the deleted pixels are reassigned to a new class value.

There were two reasons for applying the MMU filter:

- The nine-pixel "sieve" effectively removed the majority of the thematic noise in the output class maps. With high spatial resolution imagery, single pixels and small groups of adjacent pixels make up raster polygons that are very difficult to ground truth. The very fine level of detail provided by these maps is typically not required and may actually degrade the interpretability and the overall accuracy of the classification.

- Output products include ArcView shapefiles. Preliminary examination of shapefiles created from the full resolution class maps suggested that the interpretability of these vector files would be very difficult. File size was also a problem with the full resolution maps. The filtered class maps stored the data with greatly reduced file sizes and appeared to offer more easily interpreted data.

Figure 35 displays the result of the MMU filter on the Knapps Narrows class map. Employing an extremely small MMU for this project maintained the detail provided by the classified CAMIS images. 


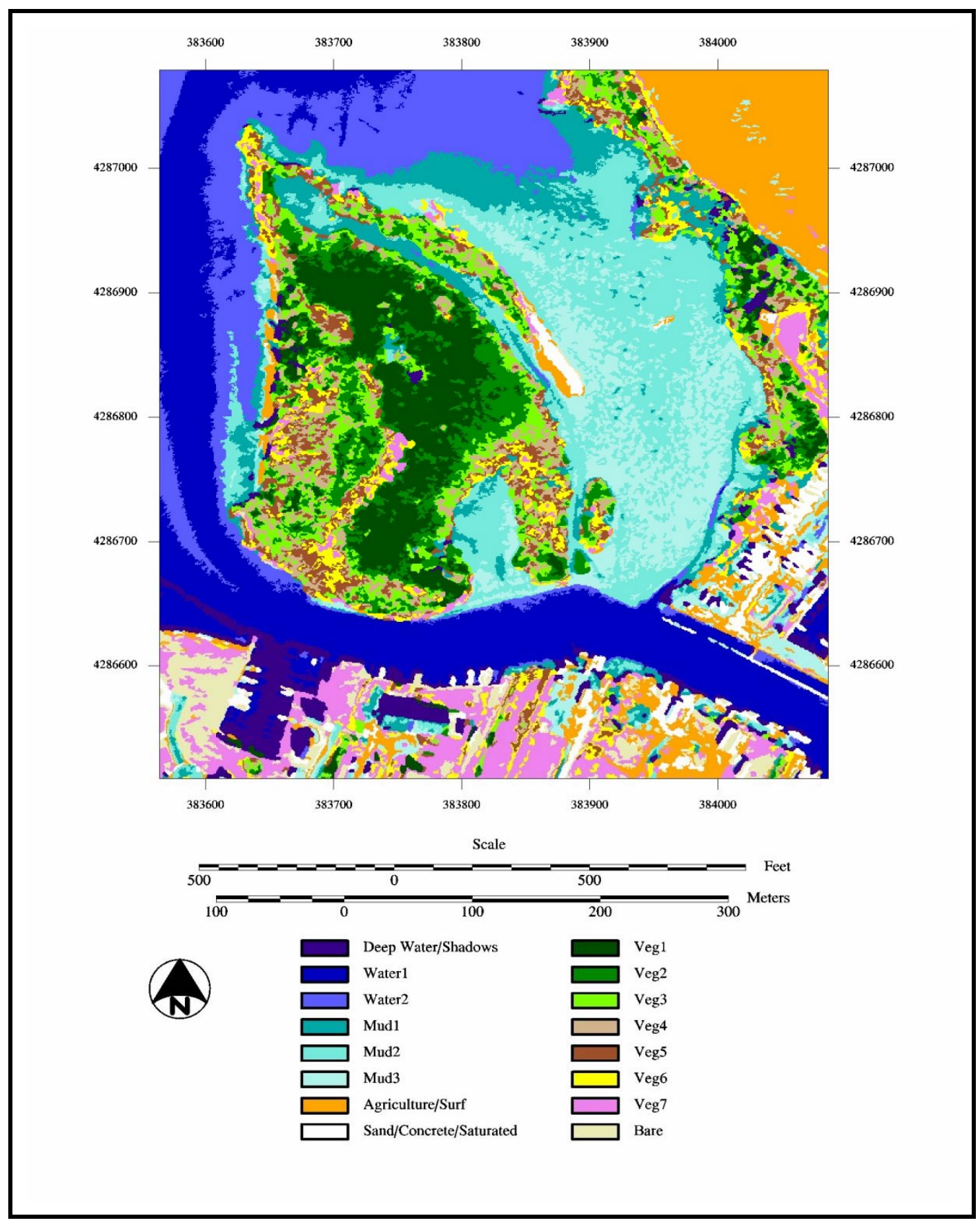

Figure 35. Class map of Knapps Narrows after application of minimum mapping unit filter (MMU = nine pixels). 


\subsection{Invasive Species Mapping at Lake Okeechobee, Florida}

Two different image processing techniques were used to develop vegetation class maps for each of the two image sources:

- $\quad$ The CAMIS airborne mosaics were processed using a supervised classification algorithm, which requires field data.

- The IKONOS satellite image was processed using an unsupervised cluster routine that did not require the use of coincident ground truth data.

\subsubsection{CAMIS Imagery}

The goal of most applications of digital remotely sensed data is to assign each multispectral pixel to predefined, discrete class (or group). This foundation for classification techniques begins with statistically based multivariate discriminant functions that determine the most likely group membership for each pixel. There are a variety of supervised classification algorithms. The maximumlikelihood classifier is one of the most frequently employed discrimination techniques. This algorithm uses the class-conditional probability density functions to calculate the likelihood that a given pixel, possessing its unique spectral vector, belongs to each of the pre-defined classes. The equation used with the maximum likelihood/Bayesian classifier is:

$$
D=\ln \left(a_{\mathrm{c}}\right)-\left[0.5 \ln \left(\left|\operatorname{Cov}_{\mathrm{c}}\right|\right)-\left[0.5\left(X-M_{\mathrm{c}}\right) T\left(\operatorname{Cov}_{\mathrm{c}}-1\right)\left(X-M_{\mathrm{c}}\right)\right]\right.
$$

Where

$$
\begin{aligned}
D & =\text { calculated weighted distance (i.e., the likelihood estimate) } \\
C & =\text { unique class } \\
X & =\text { measurement vector of the candidate pixel (i.e., the unique spectral } \\
M_{\mathrm{c}} & =\text { mean vector of class } c \text { (calculated from the training statistics for }
\end{aligned}
$$
vector) that class)

$a_{\mathrm{c}}=$ percent probability that any candidate pixel is a member of class $C$ (commonly the analyst accepts the default value of 1.0)

$\operatorname{Cov}_{\mathrm{c}}=$ covariance matrix of the pixels in class $c$ (calculated from the training statistics for that class)

$$
\left|\operatorname{Cov}_{\mathrm{c}}\right|=\text { determinant of } \operatorname{Cov}_{\mathrm{c}} \text { matrix }
$$




$$
\begin{aligned}
\operatorname{Cov}_{\mathrm{c}}-1 & =\text { inverse of } \operatorname{Cov}_{\mathrm{c}} \text { matrix } \\
\mathrm{Ln} & =\text { natural logarithm function } \\
T & =\text { transposition function used in matrix algebra. }
\end{aligned}
$$

This maximum likelihood equation, including notations and descriptions for each variable, is taken from the ERDAS Imagine Field Guide*.

There are a number of slightly different versions of the maximum-likelihood classifier published in various multivariate statistical textbooks and image processing textbooks. All variations calculate the same weighted distance estimate $(D)$ for every pixel. Actually, $D$ is calculated $c$ times for each pixel; that is, if there are five classes, then $D$ is calculated five times for every pixel. The weighted distance estimates for a single pixel are then sorted, with that pixel assigned to the class with the lowest $D$ value (i.e., the smallest weighted distance).

5.2.1.1 Signature Collection. The supervised classification algorithm must have multivariate statistics to calculate several variables in the equation above, including: $M_{c}, a_{\mathrm{c}}$, and $\operatorname{Cov}_{\mathrm{c}}$. The multivariate statistics represent a priori information and, therefore, train the classification algorithm concerning the means and ranges of pixel values that define each class. These training statistics were generated from the 36 training sites (i.e., the sample plots). Using ERDAS Imagine, we extracted pixel values (integer values for each of the four CAMIS spectral bands) from the December 2001 CAMIS images at each sample site by navigating to the $\mathrm{X}, \mathrm{Y}$ coordinates collected in the field. Typically, at least 25 pixels composed the training site. The training pixel values were stored in a separate database. Training statistics were then generated from each of the 36 sample sites. A number of plots were replicates of the same vegetation type. These statistics were combined to generate a total of 24 initial spectral signatures.

5.2.1.2 Signature Separability. Before applying the newly created spectral signatures as training data to the CAMIS mosaic, the signatures were evaluated for separability. Signature separability is a standard tool in commercial image processing software packages. The Transformed Divergence algorithm was used, although a variety of algorithms are available within Imagine. The four-dimensional mean vectors and covariance matrices are computed for the training statistics. A transformed divergence score is calculated to estimate the magnitude of the pair wise differences, or separabilities, of each of the 24 class mean vectors. The results of the signature separability tests highlighted those signatures that, while supporting unique vegetation species associations (as observed in the field), were not distinct with respect to their spectral properties. The lack of sepa-

* ERDAS (1999) Imagine Field Guide. $5^{\text {th }}$ Ed., p. 250. 
rability among several signatures suggested that different species associations with similar plant canopy architectures (e.g., height, density) maintain closely related vegetative textures. In addition, plant associations with similar amounts of senescent biomass tended to have similar spectral responses. The following bullets summarize the observations from the signature separability analyses:

- Floating mats of water hyacinth mixed with other species are spectrally similar to mixed vegetation types without hyacinth.

- Tall reeds, such as Phragmites and Typha, are spectrally similar to shorter species that have senescent biomass, including herbicide treated water hyacinth.

- Sample sites that include both green vegetation and open water are spectrally similar regardless of the species present.

- Dense mats of pure water hyacinth showed the same reflectance characteristics as dense stands of smartweed.

With nearly 5000 ha $(\sim 12,000$ acres $)$ depicted within the three CAMIS mosaics, 36 training sites (i.e., sample plots) provided a relative limited vegetation characterization.

Two options were available to minimize the negative effects associated with the inseparability of the vegetation signatures described above. One option was to aggregate the signatures to create fewer classes. This step should improve the separability of the training statistics. However, lumping classes together would reduce the potential detail in the final thematic map. A second option was to employ all 24 signatures in the maximum likelihood classifier and then combine the spectrally similar thematic classes. The second option was selected in an attempt to maintain the greatest level of spatial detail in the final thematic product.

The 24 classes in the supervised classification product were aggregated to a final 18 classes.

5.2.1.3 Minimum Mapping Unit. A minimum mapping unit (MMU) filtering routine was also applied to the full resolution class map. This filtering algorithm first delineates all of the raster polygons throughout the thematic image. A raster polygon is defined as a group of adjacent (i.e., connected) pixels with the same thematic class value. The adjacency criteria define the polygon using pixels joined along the four flat edges of the square pixel and joined at the corners. The second step is to define an appropriate MMU. For this research effort, a minimum mapping unit of 144 pixels $\left(144 \mathrm{~m}^{2}\right)$ was selected. This size is unusual because it covers the same two-dimensional area as nine 4- $\times 4$-m IKONOS pixels. The next step is to delete all raster polygons that are less than 144 pixels. This operation is synonymous with applying a polygon sieve to the thematic image, 
where all raster polygons below the MMU threshold size are "dropped." The final step in the technique is to iteratively apply a majority filter to the sieved image. This majority filter, usually a $3 \times 3$ pixel matrix, uses a neighborhood operation to fill in the now empty pixels that were lost in the sieving step. As the center pixel of the $3 \times 3$ matrix passes over each dropped pixel, the class values for the eight surrounding pixels in the matrix are tallied. The blank center pixel is then assigned the same class value as the majority of the pixels within the matrix. The filter then moves to the next sieved pixel and repeats the operation. The filter is passed over the image many times until all pixels that were deleted in the sieving operation are reassigned a new class value. The operation ignores any background pixels that were already present in the full resolution thematic map.

Figure 34 shows the aggregated vegetation class map after application of the MMU filter.

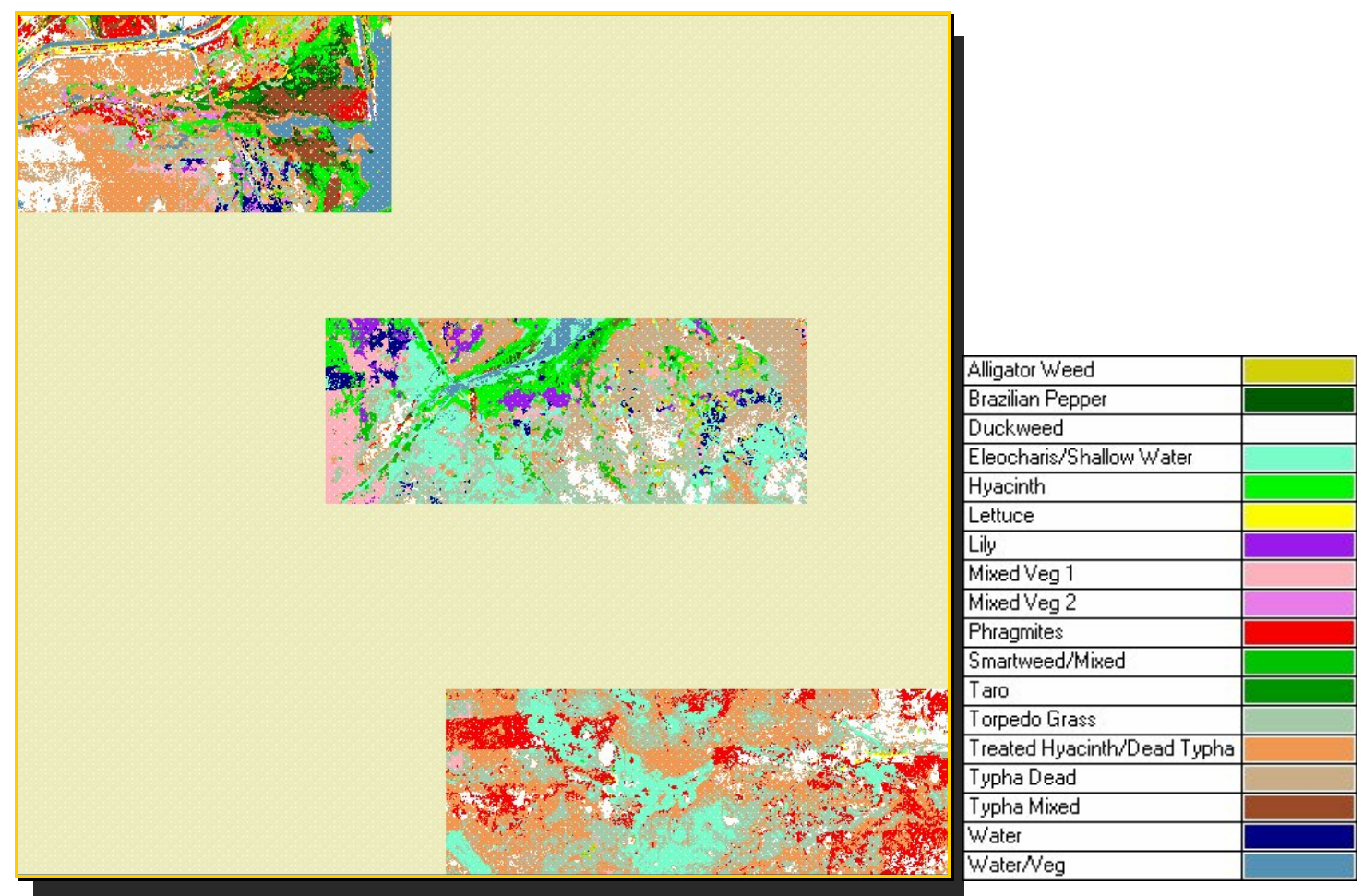

Figure 36. Supervised classification results of CAMIS mosaics after application of the 144 pixel minimum mapping unit filter. 


\subsubsection{IKONOS Image}

5.2.2.1 Histogram Matching. As stated above, no field data were acquired at the time of the acquisition of the four-band multispectral, 4-m spatial resolution IKONOS image. The classification of these data, therefore, had to rely on analyst interpretation to develop the necessary unique vegetation signatures. An attempt was made to use the signatures acquired from the CAMIS mosaics. This procedure required that the spectral histograms from the July 2002 IKONOS image be matched to the December 2001 CAMIS spectral histograms. An exhaustive analysis of the two sets of four histograms (i.e., blue, green, red, and near infrared) was unsuccessful in developing IKONOS bands with 8-bit distributions similar to those of the CAMIS bands. The primary reason for the failure of this technique is assumed to be the date of acquisition of each image set. South Florida does experience a subdued senescence of vegetation during the winter months. The CAMIS images were captured during this period. The IKONOS satellite data were collected during high summer, when the vegetation is actively growing. In addition, water levels within Lake Okeechobee significantly impact the distribution of vegetation types, particularly the areal extent of water hyacinth and water lettuce. Water levels had changed between the two acquisition dates. The differences in plant phenology and species distributions resulted in a very poor matching of the IKONOS histograms to the CAMIS histograms. The supervised classification technique was abandoned.

5.2.2.2 Unsupervised Classification. An unsupervised classification technique was employed to develop a class map from the IKONOS data. The cluster techniques applied to the IKONOS imagery over Lake Okeechobee were similar to those applied to the CAMIS mosaics for the Poplar Island Reference Wetlands Mapping project. At Poplar Island, the images were first segmented into two primary data sets using the Normalized Difference Vegetation Index (NDVI). One segment represented primarily non-vegetated surface features, while the other segment represented primarily vegetated pixels. Then each segment was classified using an unsupervised clustering routine within the Imagine software. Next, the classes from the two independent segments were labeled with vegetation or non-vegetation types using visual interpretation of the original multispectral CAMIS frames. Finally, the segments were combined and the classes were logically aggregated to create final land cover maps.

For the IKONOS scene over Lake Okeechobee, an NDVI image was again used to stratify the multispectral image, but this time into four segments. The objective of increasing the number of segments was to enhance the separability of the classes derived from the unsupervised classifications. The first segment included the lowest NDVI values and thus represented features with the least 
amount of vegetation. Examples of classes with the lowest NDVI values include: open water, low density vegetation surrounded by water (e.g., Eleocharis), and stands of dead Typha. The second segment, with NDVI values from the lower middle portion of the histogram, included low to medium density vegetation types, such as veg-water mixed and herbicide treated hyacinth. The third NDVI segment comprised pixels with medium to high density vegetation, including mixed vegetation, hyacinth-grass mix, and Phragmites. The last segment contained the brightest (i.e., greatest green biomass) vegetation pixels. Pure water hyacinth, dense areas of water lilies, and dense stands of smartweed are included in the fourth NDVI segment. A total of 18 clusters were classified within each of the four segments. The spectral signatures were plotted and, along with visual interpretation of the original multispectral IKONOS scene, were used to assign a descriptive class name to each cluster. Similar clusters were aggregated within each segment. Then, the four segments were combined, with similar clusters again aggregated. The final IKONOS derived vegetation map contained 18 thematic classes (Fig. 37).

5.2.2.3 Minimum Mapping Unit. A nine-pixel MMU threshold was applied to the IKONOS class map to remove the thematic speckle and improve the utility of the final product (Fig. 37).

\subsection{Wetland Vegetation Mapping Over Blackwater Wildlife Refuge}

The ENVI software routines for building training data sets to perform discriminant analyses, including a variety of supervised classification algorithms, extract reflectance value statistics using user defined polygons. These polygons, called Regions of Interest (ROIs), can be of any size. The objective of extracting DNs from a multi- or hyperspectral digital image is to define the $n$-dimensional statistical space unique to individual, or groups, of separable surface elements. Because training data sets must build multivariate statistics, including the mean, the standard deviation, and the accompanying covariance matrix, for each individual land cover class, ROIs generally encompass more than one single pixel. Furthermore, multiple ROIs must be used, potentially throughout the entire image space, to account for spatial variability within the scene.

\subsubsection{Image Subsetting for Wetland Vegetation Mapping Over Blackwater Wildlife Refuge}

During several preliminary image classifications and signature analyses, preformed on sets of adjacent flightlines as the larger mosaics were constructed, a number of land cover classes were identified as potentially problematic. Specifically, the agricultural fields within and adjacent to the Refuge maintain spectral 
properties very similar to several of the marsh vegetation associations of interest. Attempts to assign unique signatures to the croplands, particularly in the larger southern mosaic, proved unsuccessful. Attempts to mask (i.e., turn off) the agricultural pixels resulted in the loss of a significant number of marsh vegetation pixels. Therefore, the Refuge boundary, plus a 500-m buffer zone, was used to subset (or clip) the full southern mosaic (Fig. 38). This step removed the majority of the agricultural lands. The northern mosaic was not clipped, with the entire set of six flightlines classified into vegetation classes including five agricultural classes.

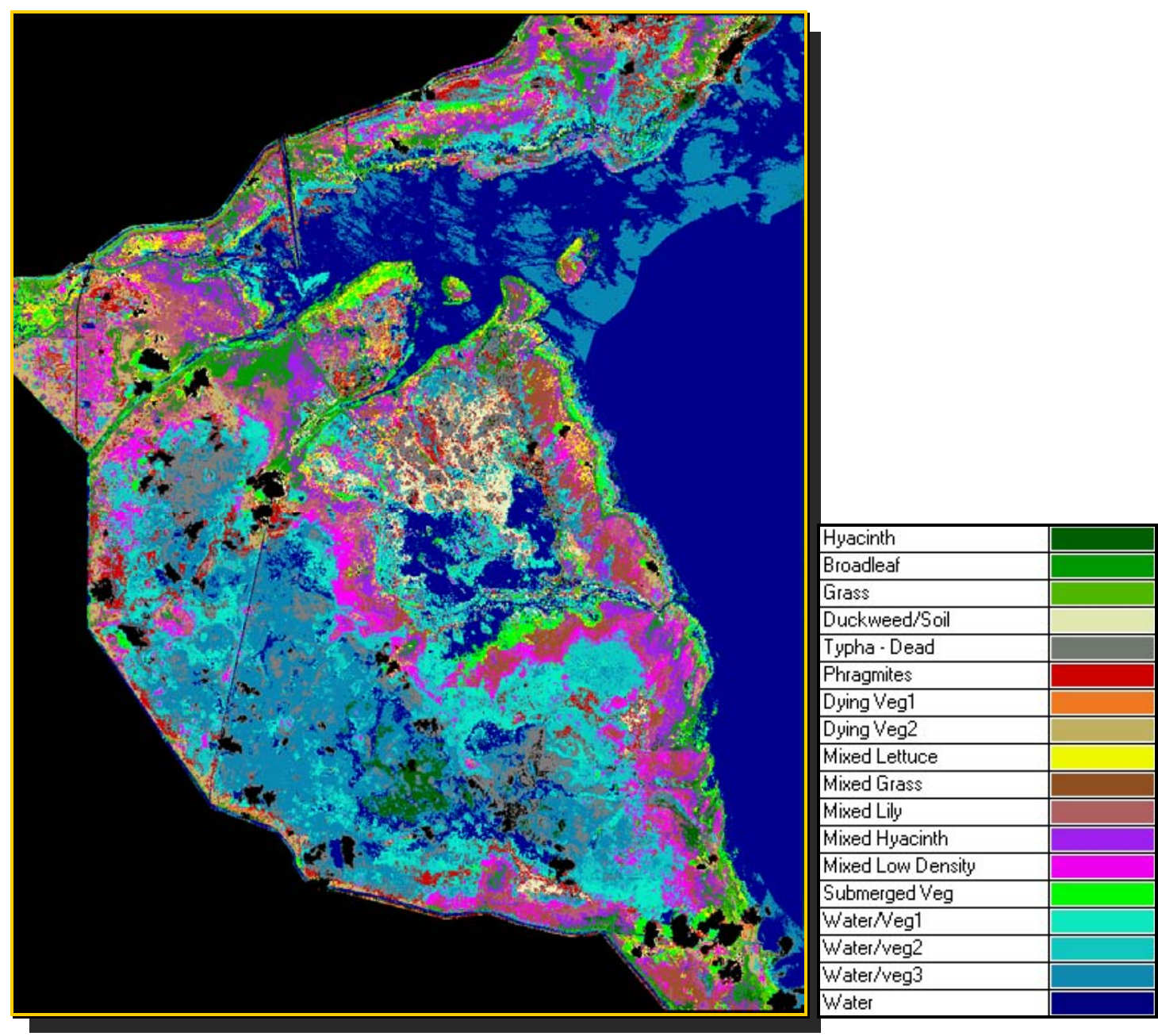

Figure 37. Unsupervised classification results of IKONOS image after application of the nine-pixel minimum mapping unit filter. 

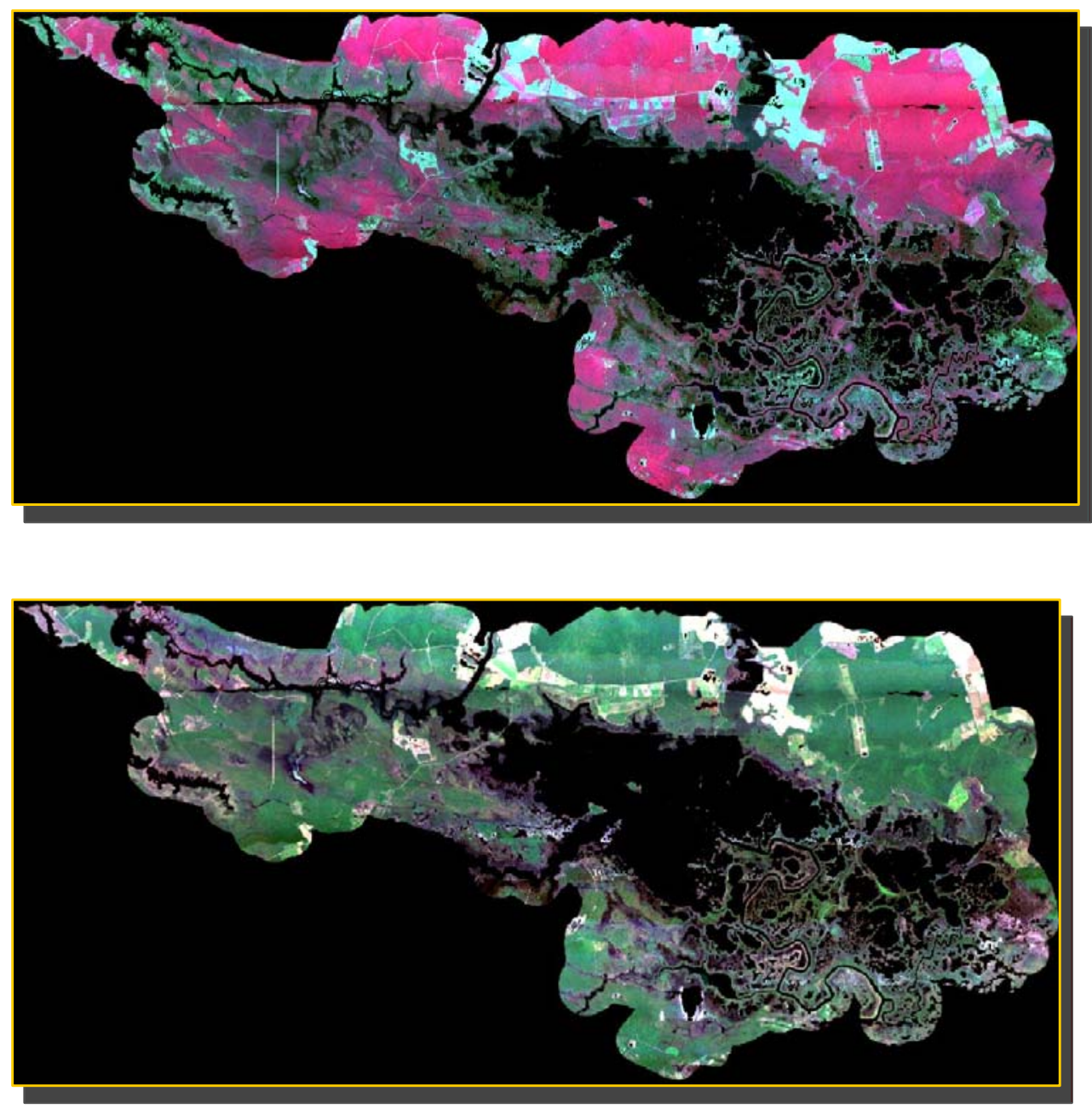

Figure 38. Southern Mosaic-flightlines BLINE7E through BLINE20E. (top) false-color composite, and (bottom) true-false-color composite.

\subsubsection{Signature Separability Analyses for Wetland Vegetation Mapping Over Blackwater Wildlife Refuge}

Before applying the newly created spectral signatures as training data to the 14-band images, the signatures were evaluated for separability. Signature separability is a standard tool in commercial image processing software packages. A variety of algorithms are generally available. ENVI offers two: Jeffries-Matusita and Transformed Divergence. Both use the $n$-dimensional mean vectors and covariance matrices of the training statistics to measure the magnitude of the difference, or separability, of the mean vectors. The Transformed Divergence statistics 
were used for these data. The algorithm calculates a separability statistic for all pair-wise combinations of signatures.

The final signatures used for classification for the southern (7 June) and the northern (30 June) mosaics, respectively, were generated by a meticulous and iterative evaluation of the class statistics for each individual training site, which identified these mean signatures as the most representative of the spectral properties within the northern and southern mosaics. During this evaluation the separability analysis was repeated many times. The output of each iteration identified the statistically indistinct training sites. Non-separable classes were either dropped from the data set if they were determined to be outliers, or merged with other training sites. The results of the last transformed divergence tests suggested that all of the pair-wise comparisons were at least moderately separable. However, because this analysis was based on an extremely small sample size (i.e., total number of pixels in each training class relative to the total number of pixels in the mosaics), it was expected that an inspection of the final class map would reveal some overlap between relative similar vegetation classes. Figures 39 and 40 display the spectral curves of the 37 class signatures used for the southern mosaic. Note the overlap of many of the signatures, particularly in the marsh grass communities.

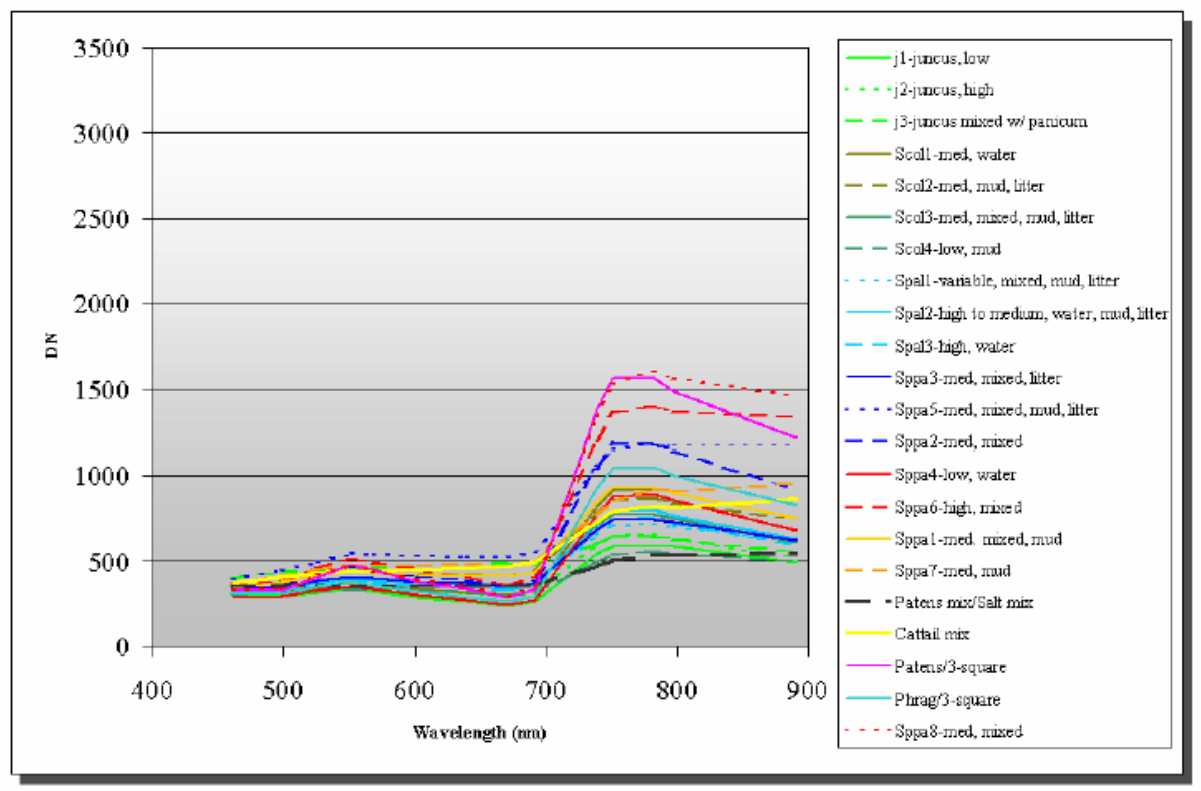

Figure 39. Spectral plots of low reflectance signatures used to classify the southern mosaic. 


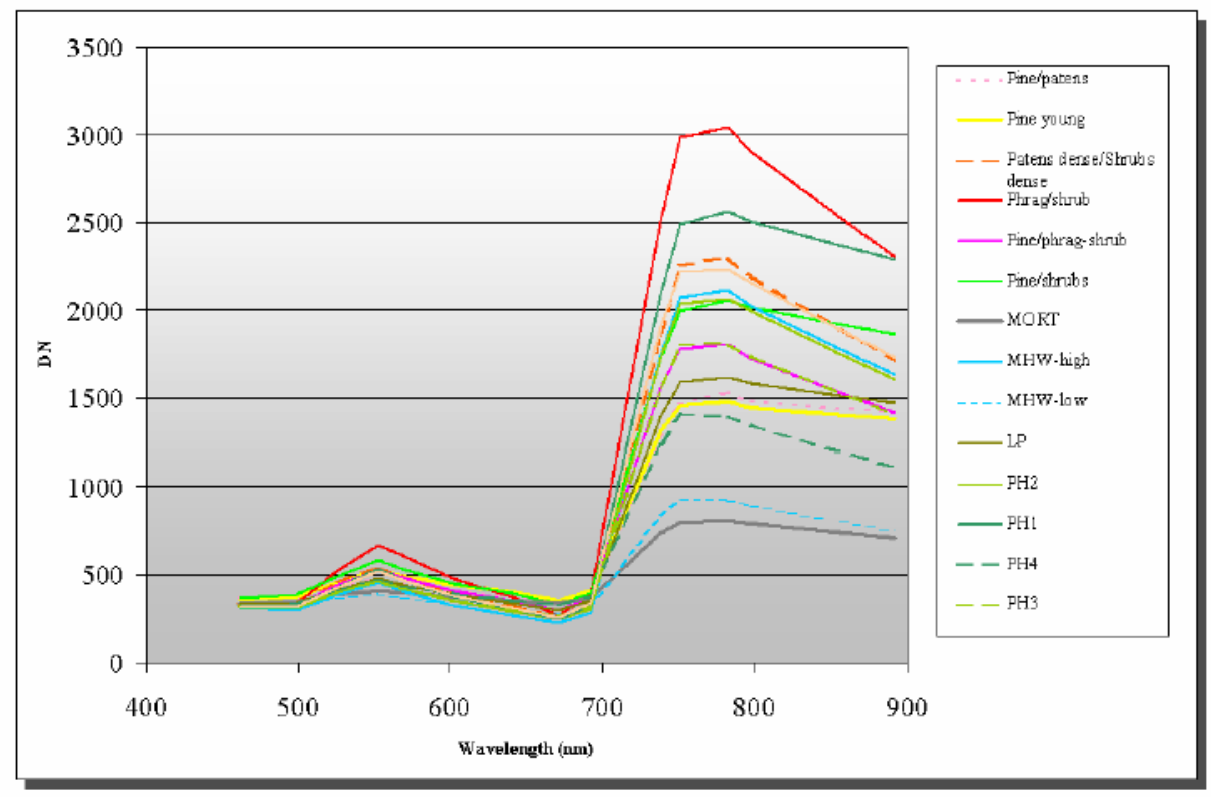

Figure 40. Spectral plots of high reflectance signatures used to classify the southern mosaic.

\subsubsection{Image Classification for Wetland Vegetation Mapping Over Blackwater Wildlife Refuge}

5.3.3.1 Spectral Angle Mapper. The images were classified using the Spectral Angle Mapper (SAM) routine within ENVI. This function assigns each individual pixel to a class based on the calculated angle, measured as the arc distance, between that pixel's DN values (using all 14 bands) and each of the mean vectors of the training signatures. The maximum spectral angle is user-defined within the routine and is given in units of radians, not degrees. For the first run of the SAM algorithm in the southern mosaic, a spectral angle of 0.08 radians $\left(\sim 4.58^{\circ}\right)$ was chosen. The 14-row, 1-column vector for the first pixel in the image (i.e., the unknown spectrum) and the 14-row, 1-column mean vector for a single reference spectrum are used as inputs into the following equation. 


$$
\alpha=\cos -1\left\{\frac{\sum_{i=1}^{n b} t_{\mathrm{i}} r_{\mathrm{i}}}{\left(\sum_{i=1}^{n b} t_{\mathrm{i}}^{2}\right)^{1 / 2}\left(\sum_{i=1}^{n b} r_{\mathrm{i}}^{2}\right)^{1 / 2}}\right\}
$$

where

$$
\begin{aligned}
\alpha & =\text { spectral angle } \\
t_{\mathrm{i}} & =\text { spectral vector of unclassified pixel } \\
r_{\mathrm{i}} & =\text { mean spectral vector of training (reference) signature } \\
n b & =\text { number of bands }
\end{aligned}
$$

The spectral angle is then calculated 37 times for each image pixel $\left(t_{\mathrm{i}}\right)$ because there are $37 r_{\mathrm{i}}$ reference signatures or classes. The pixel is then assigned to the class where the spectral angle is the smallest. If all 37 angular estimates are greater than the maximum specified angle (i.e., 0.08 radians or 4.58 degrees), the pixel is assigned to an "unclassified" group.

The results of the initial classification using the SAM algorithm showed some confusion among the vegetation classes. Also, some pixels (approximately $10 \%$ in both mosaics) were not classified. Additional SAM runs using larger spectral angles $(0.09-0.12)$ successfully increased the number of pixels that were classified. However, the confusion among classes was also increased. Therefore, the results from the 0.08 spectral angle were retained for the final class maps.

5.3.3.2 Maximum-Likelihood Classifier. A maximum-likelihood classifier was then applied to the unclassified image pixels. This algorithm uses the classconditional probability density functions to calculate the likelihood that a given pixel, with its unique spectral vector (i.e., variable $t_{\mathrm{i}}$ from the SAM equation), belongs to each of the reference classes (i.e., variable $r_{\mathrm{i}}$ ). Every pixel is then assigned to the class with the maximum probability of membership. This classification technique is used extensively with both multispectral and hyperspectral imagery.

5.3.3.3 Minimum Mapping Unit Filter for Wetland Vegetation Mapping Over Blackwater Wildlife Refuge. A minimum mapping unit (MMU) filtering routine was also applied to the full thematic resolution class maps. This filtering algorithm first delineates all of the raster polygons throughout the thematic image. A raster polygon is defined as a group of adjacent (i.e., connected) pixels with the same thematic class value. The adjacency criterion defines the polygon using pixels joined along the four flat edges of the square and those pixels joined 
only at the corners. For example, the full resolution class map for the southern mosaic had 1,541,906 raster polygons ranging in size from a single pixel to over 100,000 pixels. Both accuracy assessment and vector conversion of the thematic map is greatly hampered by so many polygons. The second step is to define a MMU. For this project, a MMU of 25 pixels $\left(225 \mathrm{~m}^{2}\right)$ was selected. At $9 \mathrm{~m}^{2}$ per pixel, 25 pixels cover only 0.0225 ha (0.0556 acres). The next step is to delete all raster polygons that have fewer than 25 pixels. This operation is synonymous with applying a polygon sieve to the thematic image, where all raster polygons below the MMU threshold size are "dropped." The final step in the technique is to iteratively apply a majority filter to the sieved image. This majority filter, usually a $3 \times 3$ pixel matrix, uses a neighborhood operation to reassign sieved pixels to a larger polygon. As the center pixel passes over each dropped pixel, the class values for the eight surrounding pixels in the matrix are tallied. The center pixel is then assigned the same class value as the majority of the pixels in the matrix. The filter then moves to the next sieved pixel and repeats the operation. The filter is passed over the image many times until all pixels within the deleted polygons are reassigned a new class value. The operation ignores any background pixels that were already present in the full resolution thematic map.

The threshold of 25 pixels was assumed to be adequate for retaining the majority of the thematic information throughout the study site, while removing a significant amount of thematic speckle (or noise) and reducing the number of raster polygons to 32,710 . 


\section{ACCURACY ASSESSMENT}

Quantitative thematic accuracy assessment is critical in determining the quality of vegetation classification. Typically, independent field data are used to calculate thematic accuracy.

\subsection{Accuracy Assessment Lake Okeechobee}

The field data sample size was inadequate to provide for a thorough assessment of the thematic accuracy of the either the full resolution or MMU filtered vegetation class maps. However, a pseudo-accuracy calculation was completed using the actual training site locations. This step is typically a preliminary procedure in assessing the quality of the thematic map. In this case, the accuracy of the 24 class, MMU filtered map was $\sim 80 \%$. The aggregated 18 class, MMU filtered map was only $\sim 70 \%$ correct. These results suggest that a number of the sample sites were incorrectly classified.

For example, the areas of mixed hyacinth are classified as Phragmites and/or Typha/Mix. Also, much of the Phragmites classified throughout Moonshine Bay (the southern most mosaic) is actually mixed vegetation. A more thorough visual interpretation of the thematic product, combined with additional analyses of the inseparability among the training signatures, suggests that the final CAMISderived vegetation class map is no greater than $60 \%$ accurate.

\subsection{Accuracy Assessment for Wetland Vegetation Mapping Over Blackwater Wildlife Refuge}

Owing to budgetary and time constraints associated with this effort, no ancillary field points were available. Instead, thematic accuracy was determined using the training data field sites. Training sites are commonly used in this manner only as a preliminary step in assessing classification accuracy.

The training sites used for classification were initially associated with very detailed vegetative parameters, including species composition, species density and background material. However, because of the relative lack of geometric accuracy in locating the $1-\mathrm{m}^{2}$ quadrats on the imagery combined with the marginal spectral separability of all of the class spectra, the final southern mosaic class map was recoded to only nine vegetation classes. The geo-registered training sites for each of the mosaics were similarly aggregated to the same classes.

Image processing software generally offers accuracy assessment routines. In this case, ERDAS Imagine was used to overlay a single point, generally the center pixel of the training area, onto the renamed class maps. The thematic class 
from the image was then compared to the true class for that point. For both class maps the overall thematic accuracy was approximately $60 \%$. This relatively low overall accuracy suggests only a marginally useful vegetation classification. 


\section{$7 \quad$ OUTPUT PRODUCTS}

\subsection{Image Quality for Poplar Island Restoration}

As described above, flying conditions were good (full sun and light winds). However, the decrease in aircraft altitude reduced the pixel dimensions (i.e., increased spatial resolution) and, more importantly, decreased the image footprint (i.e., the surface area captured within a single frame). The lower altitude did not adversely affect frame "endlap" (overlap between consecutive frames along a single flightline) but significantly decreased the "sidelap" (the amount of frame overlap between adjacent flightlines). The three flightlines covering the four sites within Marsh Creek and Muddy Creek maintained enough sidelap to adequately cover the areas of interest. However, the four flightlines acquired over Hell Hook Marsh contained "holidays" (gaps) between flightlines 1 and 2. A continuous holiday exists between flightlines 3 and 4 over Hell Hook Marsh (Fig. 41).

Owing to both leaf off conditions and the inclusion of large areas of open water, the color contrast of the features within the images ranged from very dark (e.g., water) to very bright (e.g., sand, concrete, healthy pine canopies). To compensate for the relatively low spectral response of these dark features, image acquisition settings included a longer integration time (or slower shutter speed). The increased integration time led to saturation (glaring) of some of the brighter pixels. In fact, pixel saturation was apparent during acquisition. However, the system was set to acquire using a slower shutter speed, assuming that an increase in image contrast would potentially enhance the spectral differences among the darker features. Many of the frames displayed some pixel saturation. However, the overall radiometric quality of the images was acceptable with enhanced color contrast observed throughout the water, wet soil, and senesced vegetation classes.

The greatest disappointment for the project was the exceptionally poor quality of the images acquired over Spillway 6 on Poplar Island. The extreme level of saturation within these images resulted from the highly reflective disturbed soils associated with construction activities. While not evident during acquisition, pixel saturation rendered this flightline relatively useless for land cover classification. Figure 42 shows both the false color and true color mosaic for the spillway site. 


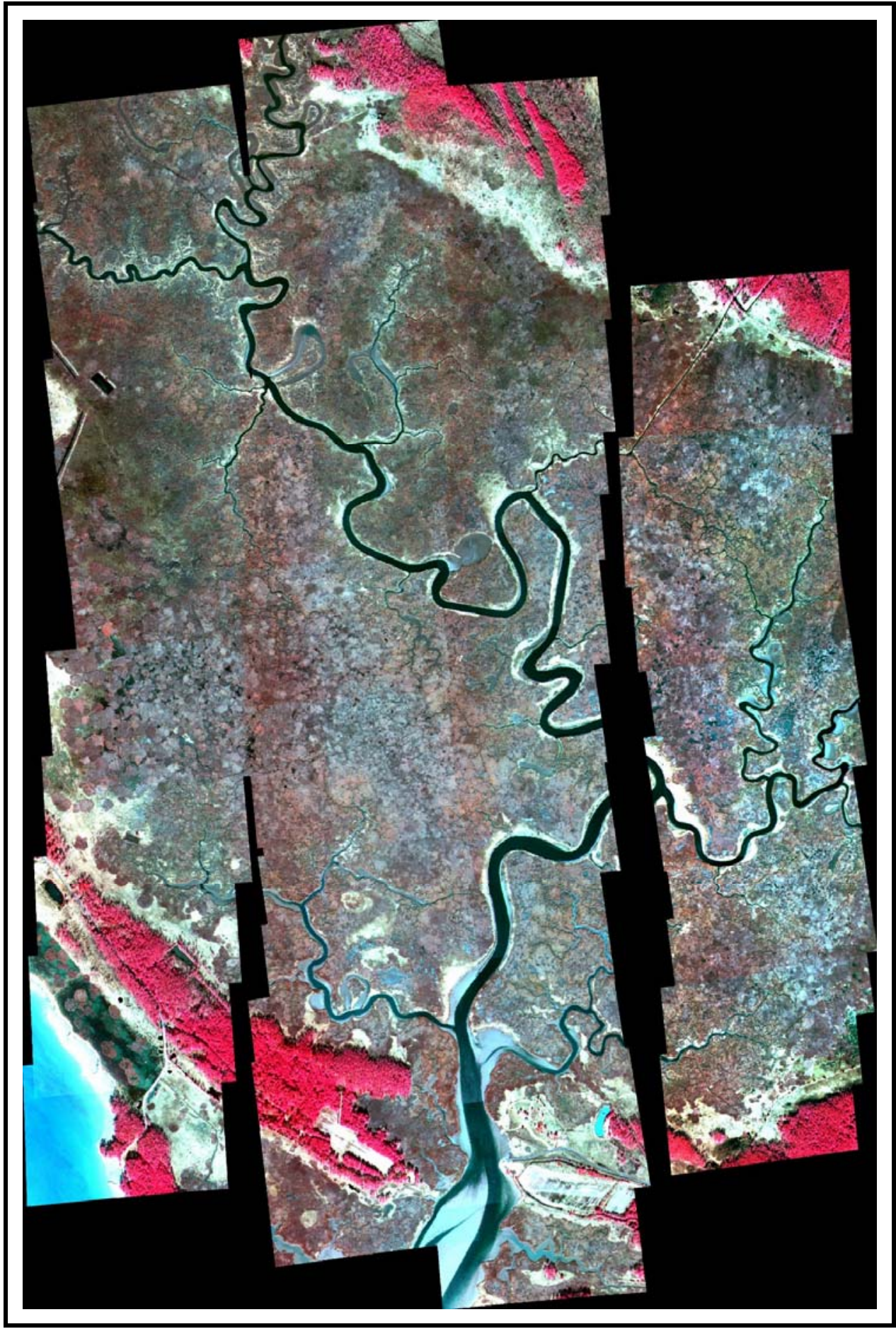

Figure 41. Mosaic of Hell Hook Marsh showing significant holidays between flightlines. 


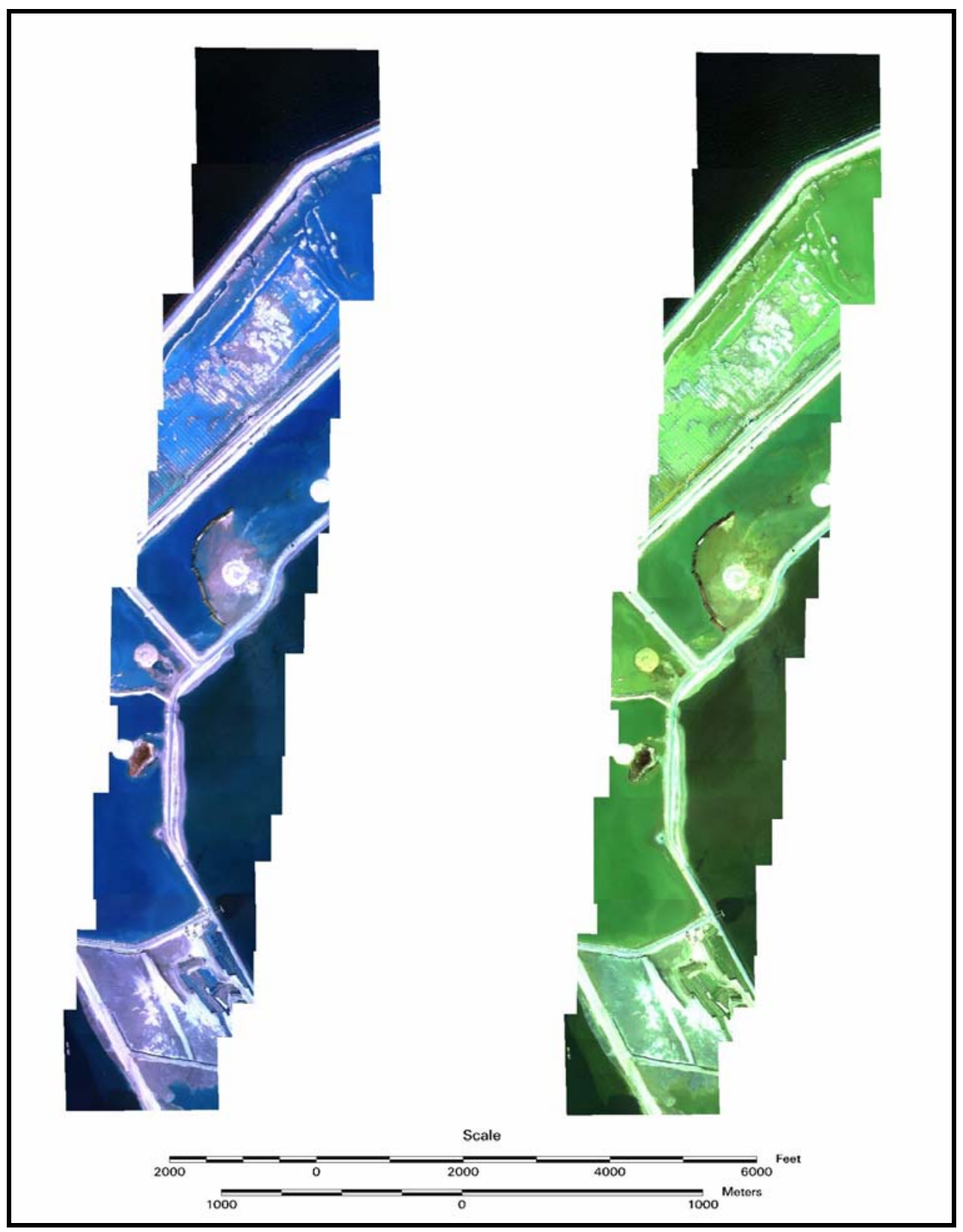

Figure 42. False color (left) and true color (right) mosaics for Spillway 6 site, within Poplar Island restoration/construction area.

\subsection{Land Cover Classes for Poplar Island Restoration}

The CAMIS image classification techniques produced detailed land cover class maps. Quantitative accuracy assessment of vegetation class identification 
would have required extensive field data collection at each site. As stated above, field data were not collected as part of the pilot project. However, based on previous CAMIS image interpretation over other coastal wetland study sites, the cover classes likely separated into the following general categories:

- Evergreen (maritime) forests.

- Tall, dense reeds.

- Short, less dense reeds.

- Marsh grasses.

- Vegetated tidal zones.

- Non-vegetated tidal zones.

- Permanent water channels of varying depth.

As described above, the assigned class names are related to spectral response (i.e., cluster spectral signature statistics) of each cluster relative to its statistically adjacent clusters. This was a subjective process. Therefore, the end user will determine the most accurate recoding of the general land categories into the three primary land cover types. Figure 43 shows the false color composite of the Cabin Creek site. Figure 44 shows the Cabin Creek class map, after application of the nine-pixel MMU threshold. The complete set of composite images and class maps are available in a companion document*.

${ }^{*}$ M.V. Campbell, R.L. Fischer, and T. Pangburn (2004) Using high spatial resolution digital imagery. U.S. Army Engineer Research and Development Center, Cold Regions Research and Engineering Laboratory, ERDC/CRREL LR-04-25. 


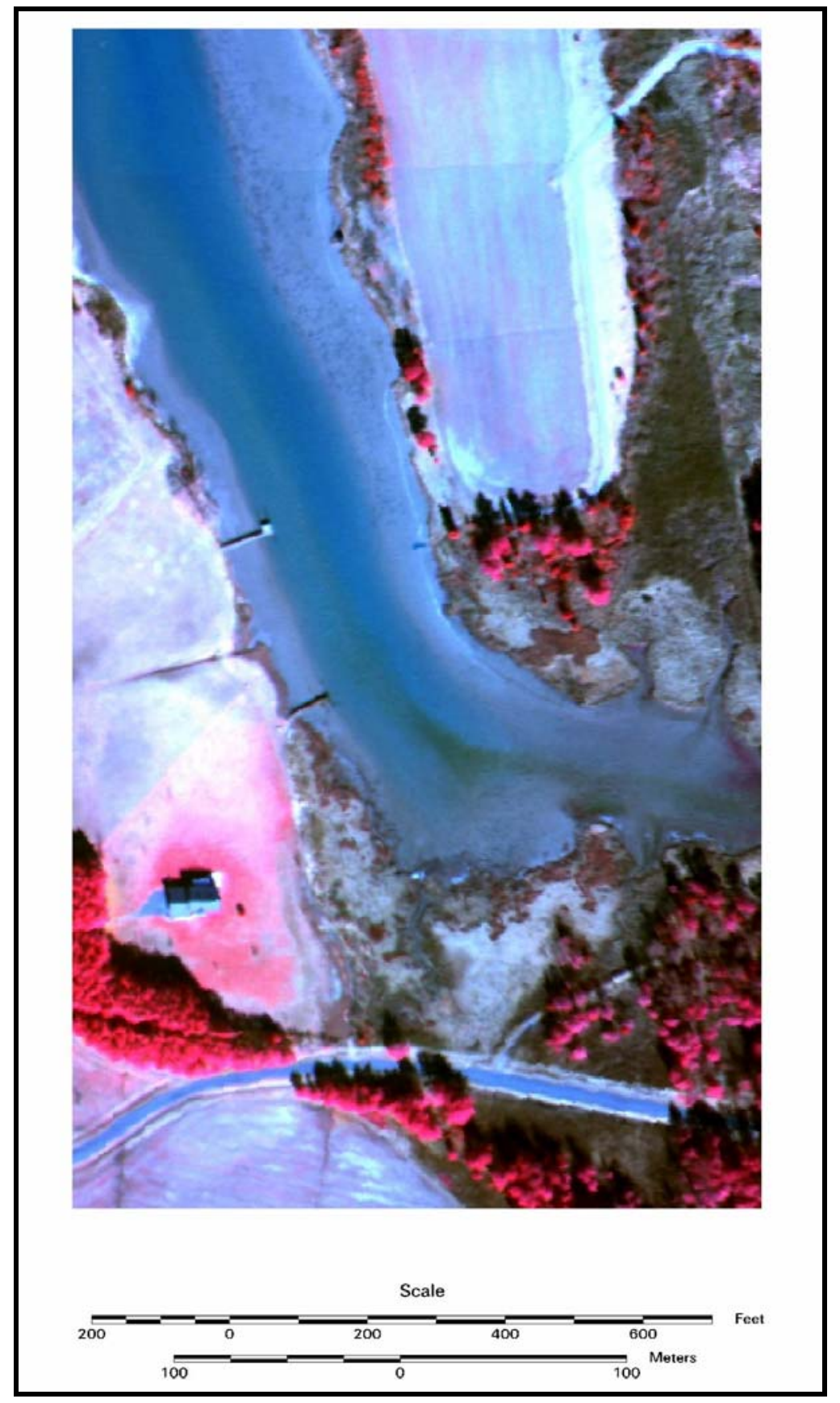

Figure 43. False color composite for Cabin Cove. 


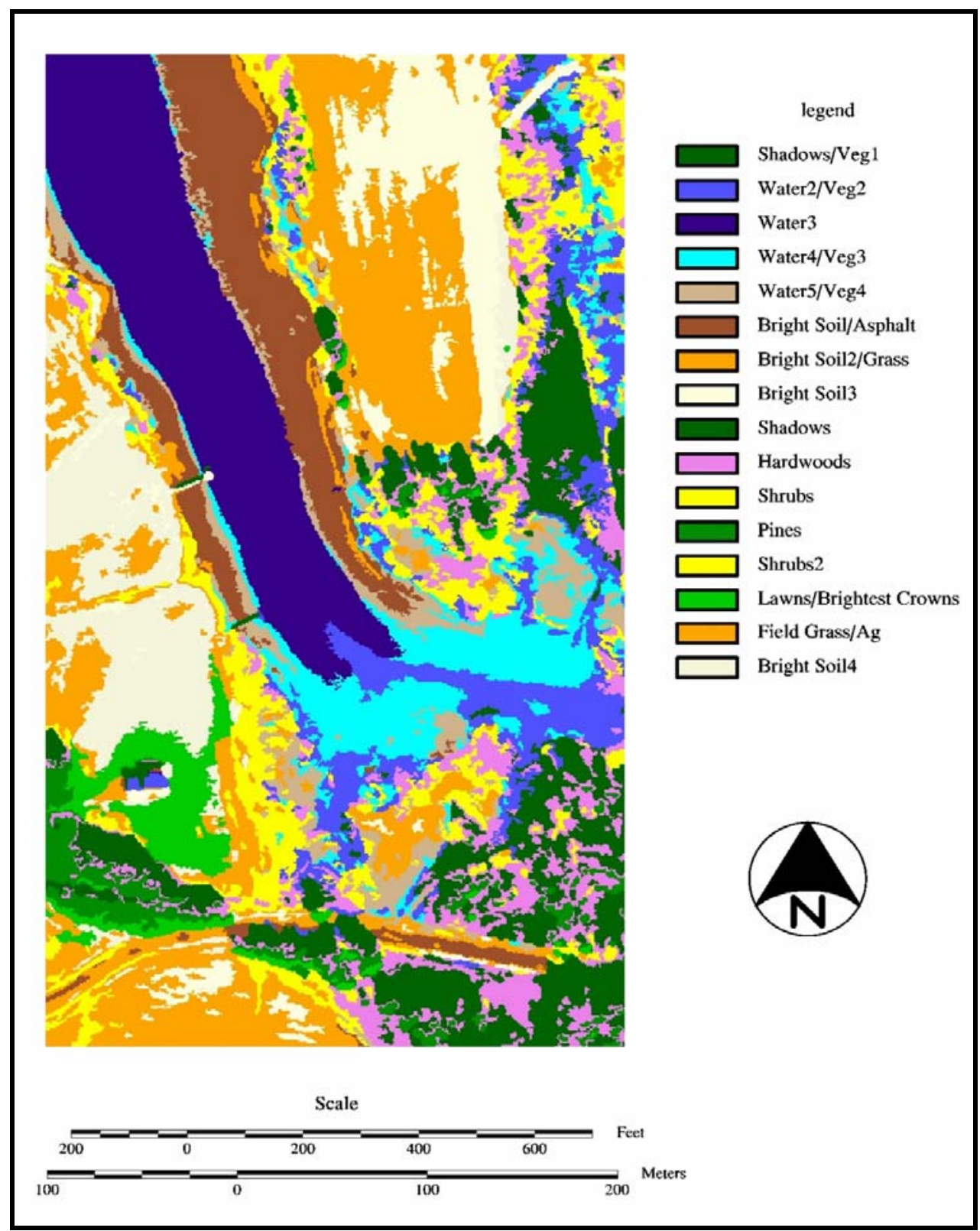

Figure 44. Class map for Cabin Cove after application of minimum mapping unit filter (MMU = nine pixels). 


\subsection{Utility For Arcview Application for Enhanced Levee Inspections}

Both sets of CAMIS mosaics were provided to the District for evaluation in assisting levee inspections. Their review was based primarily on the ability of the 1 -m multispectral mosaics to provide useful information for the inspection process. Their conclusion was that the spatial resolution would need to be significantly higher to enhance the inspections. The 1-m images depicted adequate detail for features adjacent to the levee. The detection of very small anomalies directly on the control structure, such as seepage or boils, was not reliable. This disappointing conclusion suggests that high resolution satellite data would likely not be a useful source of remotely sensed data for levee inspections. Even with application of the pan-sharpening routine, IKONOS imagery reaches its highest spatial resolution at $1 \mathrm{~m}$. The QuickBird commercial satellite, operated by DigitalGlobe (Longmont, Colorado), offers a pan-sharpened multispectral product with a $0.6-\mathrm{m}$ spatial resolution. This slightly smaller pixel is beginning to approach the desired pixel size. Communications with the District has set the desired spatial resolution at $0.25 \mathrm{~m}$ or higher (i.e., smaller than $25 \mathrm{~cm}$ ).

The CAMIS system can easily provide $0.25 \mathrm{~m}$ imagery by decreasing flying height during acquisition. However, the costs of post-processing and mosaicking approximately 2560 frames would be prohibitive. Perhaps the best source of very high resolution remotely sensed imagery would be a single-ccd digital aerial imaging system. These widely used digital cameras can produce cost-effective very high resolution imagery with excellent geometric fidelity. The primary shortcoming of the single-ced cameras is their limitation to only three spectral bands. Thus, a decision must be made to choose either true-color or false-color images. In addition, the output images are generally not suitable for advance processing such as supervised classification. This is because of the techniques required to generate three complete color planes from only one ccd array per image. Advantages provided by these systems also include typically larger focal arrays, which translate to much larger frames and fewer pictures needed to cover the area of interest. Also, when equipped with a highly precise differential GPS (dGPS) receiver, used to generate very accurate aircraft positions (X, Y, and Z), along with an inertial measurement unit (IMU), to record aircraft attitude parameters (pitch, roll, and yaw), the frames can be rapidly post-processed to produce geometrically accurate mosaics. Assuming that, for this levee inspection use, the images would be used as enhanced background data in the ArcView application, there would not be a need for a higher level of radiometric fidelity required for advanced image processing techniques. 


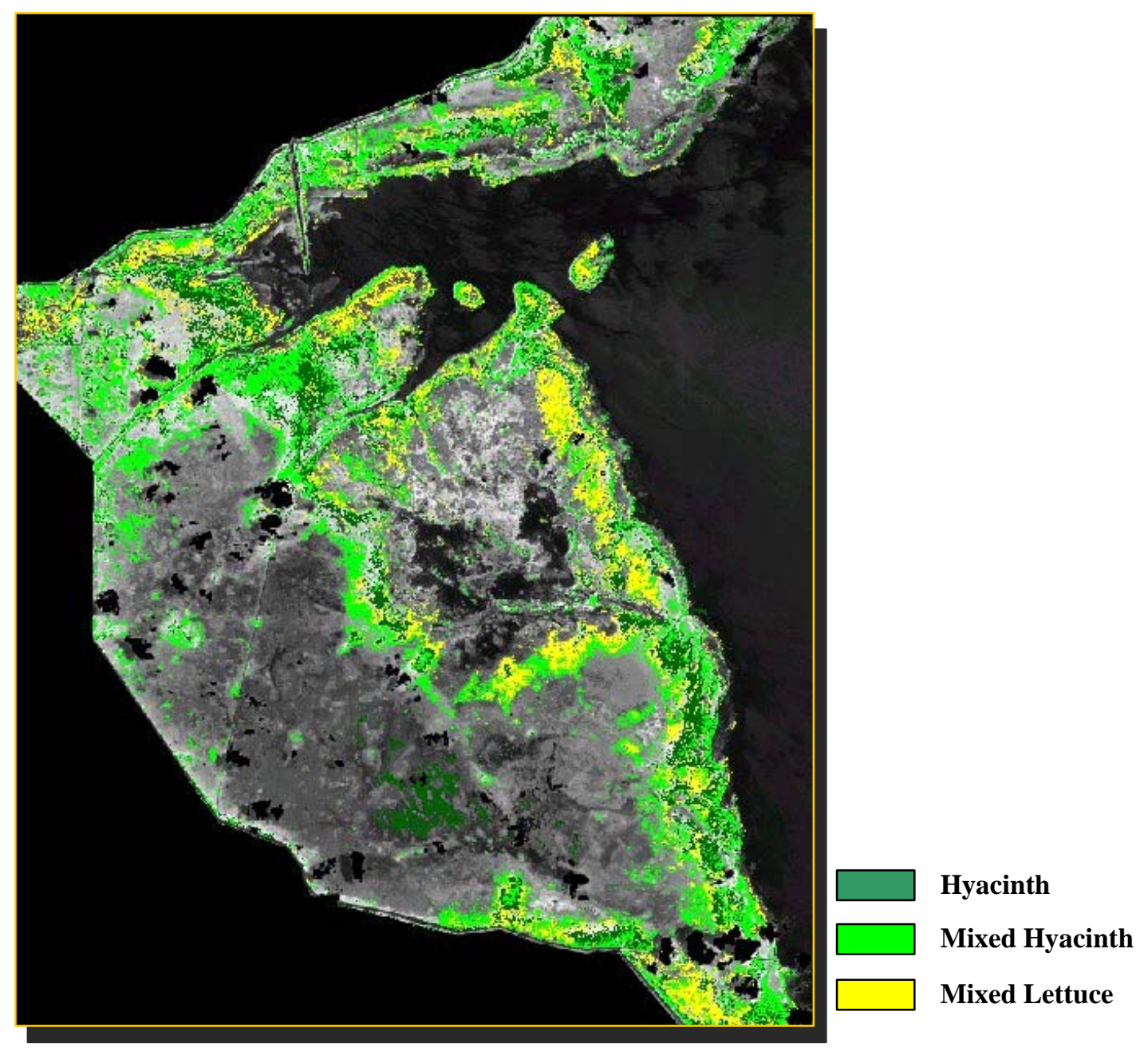

Figure 45. Final three class thematic product depicting the distribution of the invasive species of interest to the District. The background image is the near infrared channel (band 4) and is included to assist with navigation to areas supporting hyacinth and lettuce.

\subsection{Final Product for Lake Okeechobee}

The District's primary objective is to control the distribution of water hyacinth and water lettuce throughout the Lake Okeechobee. The IKONOS vegetation map, with its complete coverage of the western wetlands, may actually depict too much information for the District's needs. Figure 45 shows a three class vegetation map displayed on top of a single IKONOS spectral band. The nearinfrared band was selected as the grayscale background image. The three vegeta- 
tion classes were aggregated from the complete set of 18 classes. The objective of this product is to provide the District, Field Office, and spray contractors with the optimal level of vegetative information needed to direct herbicide application methods. This two layer map would be stored in a digital format and provide valuable spatial information for seasonal (or long-term) monitoring of hyacinth and lettuce growth and movement within the Lake. The map could also be plotted to a large map sheet and provided directly to spray crews with the intent of increasing the efficacy of herbicide application.

\subsection{Results for Wetland Vegetation Mapping Over Blackwater Wildlife Refuge}

The results of the SAM classification and the maximum likelihood classification were combined to create the complete vegetation class maps. An evaluation of the 37 classes associated with the southern mosaic indicated significant confusion within and between the vegetation types, particularly within the marsh communities. Therefore, a number of classes were combined, based on the spectral and spatial characteristics of the class signatures in the completed thematic map. A simple GIS operation was used to recode (or renumber) classes that were spectrally similar. The resulting vegetation map depicted 26 vegetation classes. The northern mosaic maintained 19 vegetation classes. Figure 46 shows the 30 June (southern mosaic) class map after application of the minimum mapping unit filter. Figure 47 shows the 7 June (northern mosaic) class map after filtering and subsetting with a 500-m buffer around the Refuge boundary. 


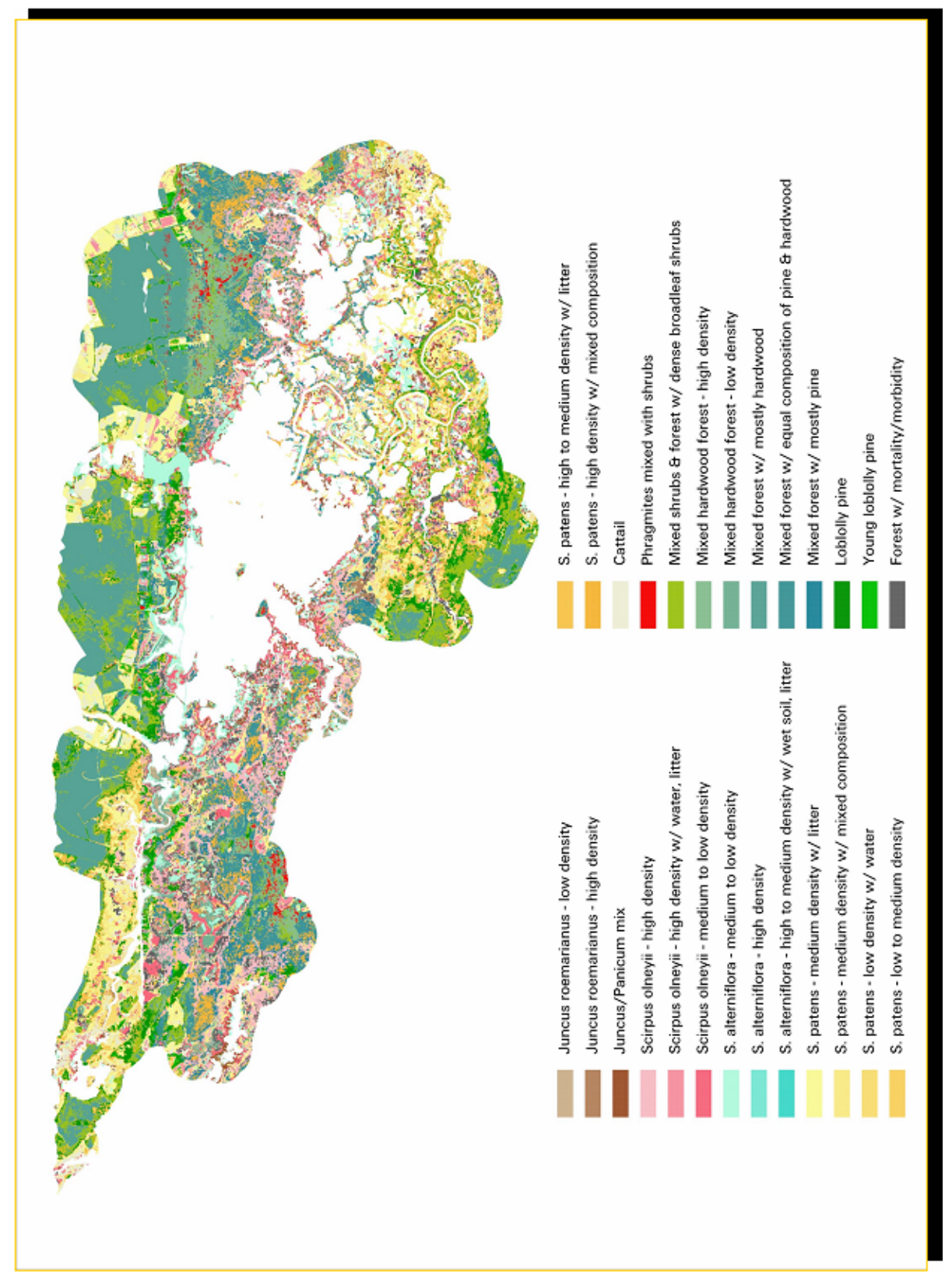

Figure 46. Final vegetation class map for southern mosaic (7 June 2000). 


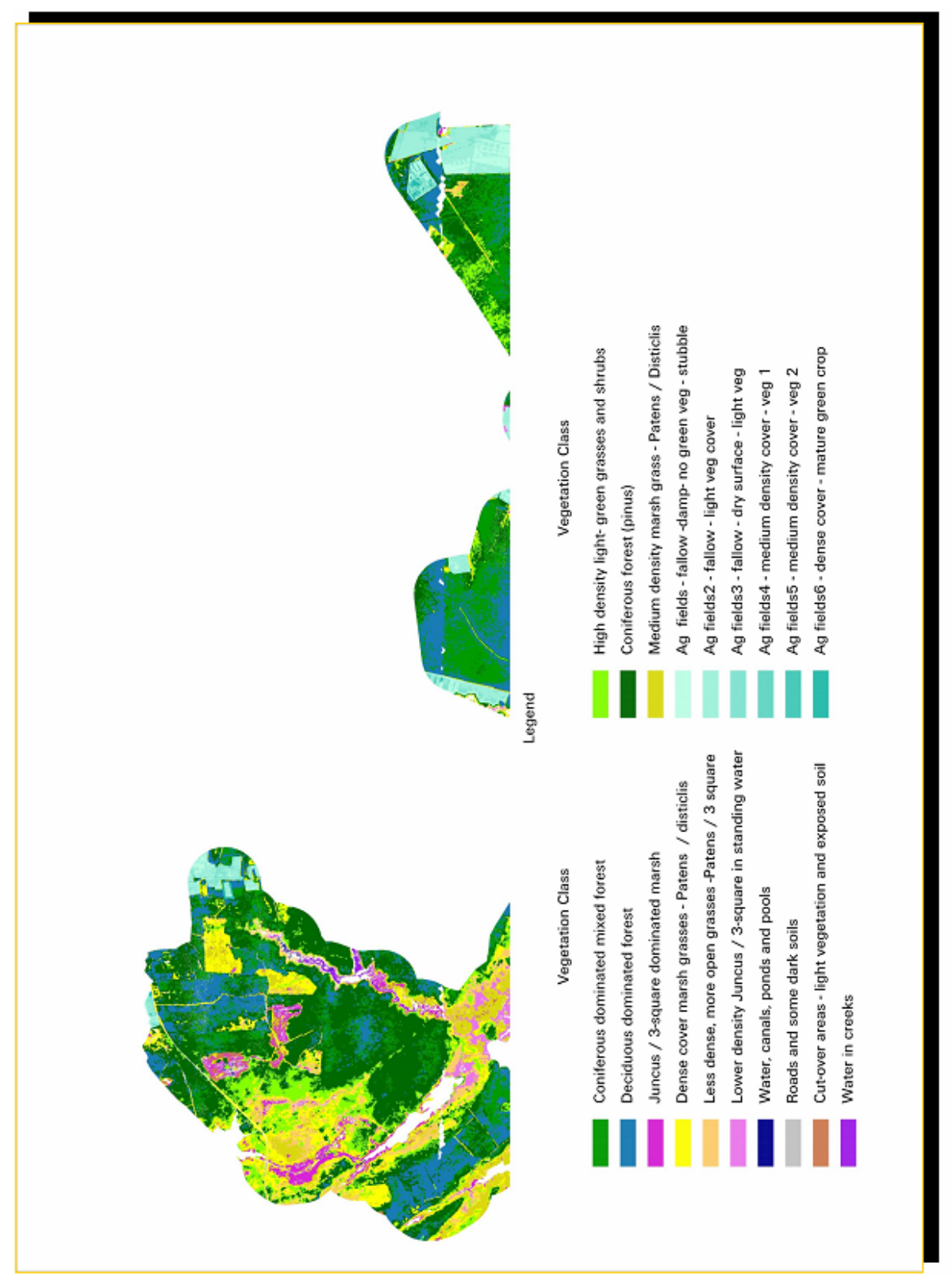

Figure 47. Final vegetation class map for northern mosaic (30 June 2000). 


\section{COSTS}

\subsection{Poplar Island Restoration}

The overall cost of this small effort was approximately $\$ 20,000$. This total included: mission planning, image acquisition, image post-processing and image classification. Owing to the fragmented locations of the eight individual study sites, the per unit area cost is relatively high at $\$ 10$ to $\$ 15$ per acre. However, high resolution satellite data, such as IKONOS imagery, would not have been more cost effective. This is because of the minimum area requirements defined by the vendors. It is estimated that three commercial satellite images would be required to cover all the reference wetlands, each costing approximately $\$ 10,000$. When combined with processing costs, the total estimated price to produce these 1 -m resolution class maps using satellite imagery would be between $\$ 40,000$ and $\$ 50,000$.

\subsection{Enhanced Levee Inspections}

Project costs can be segregated into: 1) image acquisition and 2) image processing. Image acquisition costs differed slightly between the two collection dates. The September 2001 acquisition employed a CAMIS sensor owned by TEC (originally purchased from Flight Landata in 1998). TEC contracted with a local flight services company, with TEC personnel operating the CAMIS system during acquisition. Owing to a number of unavoidable constraints, image capture was limited to a 2-day window. The total mission costs were roughly $\$ 12,000$, including $\$ 8000$ for flight services and $\$ 4000$ for labor and travel. Image postprocessing costs, including data archiving, geometric/radiometric corrections, and data transfer, are estimated at approximately $\$ 18,000$. This estimate assumes a rate of $\$ 80 /$ hour for 225 hours. Therefore, the total cost for creating the relatively poor mosaics was $\sim \$ 30,000$. The failure of this initial mission can be attributed to the limited time allowed for image acquisition coupled with the poor atmospheric conditions.

Flight Landata, using their CAMIS equipment (identical to TEC's sensor), acquired the July 2002 image data. Because their contract stipulated cloud-free imagery, the contractor did not set a rigid schedule and timed the image acquisition with favorable weather conditions. The total cost for the second acquisition mission was $\$ 20,000$. Post-processing costs, assuming the same number of hours and the same labor rate, remained at $\$ 18,000$. Therefore, the total cost of the more successful second mission was $\sim \$ 38,000$. The CAMIS frames cover ap- 
proximately $44 \mathrm{~km}^{2}$ ( $\sim 11,000$ acres). Using an average of the total estimated costs for the two missions, including acquisition and processing (given above) gives a price per square $\mathrm{km}$ of roughly $\$ 750$. This converts to approximately $\$ 3.00$ per acre.

The CAMIS images have a much higher cost per unit area relative to high spatial resolution satellite imagery. The vendors of high resolution satellite imagery require a minimum area for purchase. Using IKONOS imagery as an example, Space Imaging (Thornton, Colorado) has set the minimum area at $10 \times 10$ $\mathrm{km}$. The price for this minimum window, assuming only basic geometric registration (i.e., not ortho-rectified), is roughly $\$ 55 / \mathrm{km}^{2}$ or $\$ 5500$. This price includes both 1-m panchromatic and 4-m multispectral data. A rectangle measuring $20 \mathrm{~km}$ wide (east/west) by $25 \mathrm{~km}$ long (north/south) is required to cover the Sny levee. Therefore, cost for acquiring high resolution IKONOS imagery for this $500-\mathrm{km}^{2}$ area would be approximately $\$ 27,500$. The price tag for acquiring and postprocessing the same $500-\mathrm{km}^{2}$ area using 1-m CAMIS imagery would exceed $\$ 375,000$. The satellite data are much cheaper and generally do not require significant radiometric or geometric corrections. The IKONOS imagery must be run through a "pan-sharpening routine." The process, available as built-in routine in both ENVI and Imagine software packages, merges higher resolution (e.g., 1-m pixels) panchromatic imagery with slightly lower resolution (e.g., 4-m pixels) multispectral data to produce a simulated 1-m multispectral product. These images are excellent for manual interpretation but, with questionable radiometric fidelity, may not always be suitable for classification with quantitative image processing routines.

Compared with traditional aerial photography, digital imaging systems have several cost effective advantages. The primary benefit of CAMIS-like systems is that the raw data are provided in a digital format. Aerial photos must be transformed, through scanning, from an analogue format (either paper or transparency) to a digital format. The scanned aerial frames can then be post-processed as digital files, requiring similar radiometric and geometric post-processing steps as with CAMIS frames. The second benefit of multi-camera digital systems is their ability to acquire more than three spectral bands. The aerial photo mission would have to be flown twice, with appropriate film and camera lens, to provide both true-color and false-color images. A third benefit is total time needed to provide a completed digital mosaic. Assuming that the appropriate hardware and software tools are available, the multispectral imaging system could produce a radiometrically balanced, geometrically corrected mosaic within 2 weeks. The analogue photos require film developing and digital scanning just to prepare the imagery for the digital post-processing steps. These steps are completed twice to produce mosaics with more than three spectral bands. Assuming that the film developing 
and scanning adds one additional dollar per acre over the cost of the CAMIS imagery (i.e., $\$ 4.00$ per acre) gives a total cost to produce a true-color digital mosaic of approximately $\$ 44,000$. The false-color mosaic would double the price to $\$ 88,000$.

The one advantage that scanned hardcopy photos have over the CAMIS imagery is the potential to produce digital frames with very high spatial resolution. Depending on the original scale of the photography, the pixel dimensions of the scanned print can be very small (e.g., less than $0.25 \mathrm{~m}$ ). CAMIS imagery can also be acquired with extremely high spatial resolution by lowering the altitude of the aircraft. A decrease in pixel size results in a decrease in the areal coverage of each frame. The addition of frames needed to fully cover the site requires larger digital file sizes. For example, to increase the spatial resolution from 1 to $0.5 \mathrm{~m}$, the total number of frames required to cover the same area increases by a factor of four. To further increase the resolution to $0.25 \mathrm{~m}$ requires 16 frames for every frame with 1-m pixels. The costs of post-processing and mosaicking these higher resolution imagery increases at the same exponential rate, but would easily double if pixel dimensions were decreased to $0.5 \mathrm{~m}$ and would more than double with the decrease to $0.25-\mathrm{m}$ pixels.

\subsection{Invasive Species Mapping at Lake Okeechobee, Florida}

The cost to acquire the December 2001 CAMIS frames was $\$ 20,000$. Image post-processing and mosaicking costs were estimated at approximately $\$ 16,000$ (200 hours at $\$ 80 /$ hour). Field data collection costs, including travel and labor, are estimated at $\$ 10,000$. Image classification required another $\$ 6400$ (80 hours at $\$ 80$ /hour). At a total cost of $\$ 52,400$, the completion of the CAMIS vegetation classification required roughly $\$ 10 /$ ha $(\$ 4.50 /$ acre $)$.

The IKONOS image acquisition costs were $\$ 33,000$. With no post-processing steps necessary, image analysis was focused on developing the vegetation class map. At total of 80 hours were used to develop the final map, for a total cost of $\$ 6400$. The 4-m satellite image covers roughly 35,000 ha (85,500 acres) of marshland. At a total cost of almost $\$ 40,000$, the IKONOS class map cost little over $\$ 1$ per hectare $(\sim 0.50 /$ acre $)$.

The total costs for both of the vegetation class maps reflects an inadequate amount of field data collection. The IKONOS data were processed without any concurrent on-site observations. Based on the cost to visit the limited number sample sites used to classify CAMIS mosaics, a more thorough field data campaign would require at least three times the sample size. The number plots would again increase to collect an adequate number of accuracy sample sites within the CAMIS mosaic areas. The IKONOS image, covering seven times the area shown 
by the CAMIS frames, certainly would require an extensive network of field plots, distributed over the entire 35,000 hectares, to produce a reliable thematic product. However, remembering that the District is primarily interested in mapping the distribution of only two specific species, the number of field observations could be significantly reduced.

Perhaps as a general "rule-of-thumb," this investigation at Lake Okeechobee suggests that the project budget for creating a reliable vegetation map from high resolution multispectral imagery should include roughly equal dollars for image acquisition and field data collection. Reliability is quantified for this remote sensing application as an overall thematic accuracy of approximately $80 \%$. Image post-processing (if required) and image analysis costs, including supervised or unsupervised classification, should be included as separate items.

\subsection{Wetland Vegetation Mapping Over Blackwater Wildlife Refuge}

The costs associated with this project started with a reasonable sum for image acquisition. The vendor, 3Di, LLC captured and post-processed over 9 GB of hyperspectral images, covering 30,000 acres of Refuge property, for only $\$ 20,000$. However, as described above, a significant amount of additional postprocessing was required to create a marginally useful mosaic. The total additional cost for developing this mosaic and producing the final class map was in excess of $\$ 100,000$. The contactor should be responsible for these tasks and should be able to provide a detailed, and understandable, description of all acquisition and post-processing techniques and algorithms as part of the required metadata documentation.

A comprehensive set of ground truth sample sites would likely have cost another $\$ 25,000$ to $\$ 50,000$. When compared with traditional aerial photography, the digital imagery is initially cheaper to acquire and process. Furthermore, the digital imagery will likely have a much quicker delivery as compared to analogue photography. However, the questionable accuracy of the thematic map suggests that for projects where very detailed vegetation classification is required (i.e., species alliances or associations), large scale true-color or false-color photography, combined with intensive field data collection, may be the most cost effective alternative. Digital aerial mapping camera technologies are also maturing rapidly and may represent an alternative to hardcopy photography. While not specifically designed or suited for computer-aided classification, very high-resolution digital photos can provide an excellent source of interpretable images with very good geometry (i.e., spatial accuracy) and with a very rapid turn-around time.

Future attempts to develop a useable base map for an area as large as the Blackwater Wildlife Refuge should employ either medium or high-resolution 
multispectral satellite imagery. Medium resolution sensors include the latest SPOT and Landsat systems. These sensors provide 10 - to $30-\mathrm{m}$ pixels, respectively. SPOT and Landsat also provide 5- and 15-m panchromatic (i.e., black and white) imagery, respectively. The process of combining the higher resolution panchromatic pixels with the larger multispectral pixels is called pan-sharpening. The result is a multispectral image with the higher (i.e., small pixel size) panchromatic spatial resolution. Costs for pan-sharpened medium resolution imagery covering the Refuge will be in the $\$ 10,000$ to $\$ 20,000$ range. This price includes radiometric post-processing to remove internal sensor distortions and anomalies, and geometric registration equivalent to national map accuracy standards for 1:24000 USGS topographic quadrangles. These satellite data sources should provide an adequate source to delineate vegetation to the subgroup or formation level as defined by the National Vegetation Classification Standard.

Current high-resolution satellite sensors are IKONOS and QuickBird. IKONOS acquires 4-m multispectral pixels and 1-m panchromatic pixels. QuickBird collects 2.8-m multispectral pixels and 0.7-m panchromatic pixels. Both sources maintain price ranges from $\$ 30,000$ to $\$ 40,000$ to cover an area as large as the Refuge. As with the medium resolution image, these high-resolution sources are provided with radiometric and geometric post-processing already completed. 


\section{CONCLUSIONS AND RECOMMENDATIONS}

Each of the four study sites demonstrated the potential use of high spatial resolution digital remotely sensed imagery for a specific application. Successes and shortcomings were presented for each application. The following broad recommendations provide guidance in the effective and efficient application of highresolution imagery.

- The total size of the project site or sites, measured as the total study area (acres or hectares), will be one of the primary factors in determining the type of high-resolution imagery to acquire. For areas less $50 \mathrm{~km}^{2}$, airborne imagery may provide the most cost effective image source. For areas of interest approaching $100 \mathrm{~km}^{2}$, high-resolution satellite data will be the cheapest solution.

- When contracting the acquisition of airborne digital imagery, either multispectral or hyperspectral, ensure that the contractor provides accurate and comprehensive metadata describing the precise acquisition parameters, as well as the tools and techniques used to radiometrically and geometrically post-process the images. Generally, expect higher image post-processing costs for hyperspectral imagery versus multispectral imagery. If the contractor is to develop a map product, require an exhaustive report that documents all ground truth data collection procedures and image processing routines, including a statistically valid accuracy assessment.

- Ensure that an adequate volume of reliable ground truth data is collected and analyzed to meet the objectives of the mapping component of the overall project. Roughly plan on equal dollar amounts for image acquisition and ground truth data collections costs. Image post-processing, image classification, accuracy assessment, and report generation costs will be determined by the overall size of the project site.

\subsection{Poplar Island Restoration}

Based on the overall quality of the imagery in terms of spatial resolution, radiometric balance, geometric accuracy, and land cover classification, the objectives of this pilot project were met. The ArcView shape files should provide the Poplar Island wetlands design team useful information concerning the areal coverage, distribution, and configuration of the three primary surface features (i.e., water, intertidal zone, and vegetated wetlands). Of the 10 reference wetland areas imaged, eight sites provided land cover class maps. 
The images were acquired near low tide during a leaf-off season. It is recommended that follow-on work acquire remotely sensed data during the growing season and near high tide conditions. Furthermore, the smaller reference wetlands, while easily accessible from the ground, may not offer the variation in surface features seen in the much larger Hell Hook Marsh. It is also recommended that future remote sensing applications center on Hell Hook Marsh and other large, diverse estuarine wetlands. Ground-truth data will be required to complete a quantitative image classification and an objective land cover class map accuracy assessment.

\subsection{Enhanced Levee Inspections}

This application of high-resolution imagery to enhance levee inspections showed that 1-m multispectral imagery was of only marginal utility. The District's evaluation clearly showed a need for even smaller pixels. It is recommended that a single-CCD digital aerial camera system be employed to acquire data with very high spatial resolution (e.g., $0.25 \mathrm{~m}$ ). The estimated costs to receive a complete mosaic covering the $45 \mathrm{~km}$ ( 28 miles) of the Sny levee, including an acceptable buffer region of $500 \mathrm{~m}$ on each side of the structure, would be between $\$ 30,000$ and $\$ 40,000$.

\subsection{Invasive Species Mapping at Lake Okeechobee, Florida}

This project expended a significant amount of planning and execution to acquire the airborne multispectral imagery. The satellite imagery offered a much more cost effective data source for mapping the relatively large study area. The District was periodically updated throughout the project. Their evaluation of the thematic maps suggested that remotely sensed imagery could potentially offer a cost effective source for monitoring hyacinth and lettuce distributions across all of Lake Okeechobee. A rough estimate of the cost of acquiring 4-m IKONOS imagery over the entire Lake was well over $\$ 100,000$. A less expensive image source was identified as the SPOT sensor. With 10-m multispectral and 2.5-m panchromatic imagery, a pan-sharpened four-band product would cost between $\$ 20,000$ and $\$ 25,000$. The price range assumes that some post-processing will be required to radiometrically balance (or equalize) the several SPOT scenes required to cover all of Lake Okeechobee. Image classification costs, including the collection of the required minimum amount of field data, would be reduced by employing an unsupervised clustering routine to identify hyacinth and lettuce pixels. Field data collection could then be limited to verification of areas mapped as hyacinth and lettuce. Depending on the available budget, SPOT imagery could be acquired and processed seasonally (i.e., three to fours times per year), provid- 
ing valuable invasive species monitoring data by mapping the distribution of hyacinth and lettuce over the whole of Lake Okeechobee.

\subsection{Wetland Vegetation Mapping Over Blackwater Wildlife Refuge}

The objective of this image processing research effort was to create an accurate thematic map depicting detailed vegetation classes throughout the Blackwater Wildlife Refuge. A preliminary evaluation of the AISA hyperspectral imagery suggested that association level classification would be feasible, considering the high spatial resolution $(3 \mathrm{~m}$ ) and the excellent geo-registration of the 20 flightlines needed to cover the Refuge. However, as the individual flightlines were assembled to form a complete mosaic, serious radiometric problems were discovered. Much of the research effort was spent trying to remove a significant crosstrack illumination anomaly and attempting to match, or balance, the histograms of adjacent, overlapping flightlines to remove the visible seams. The final mosaic was of acceptable quality for image classification, but retained some radiometric distortions, including several significant seams and a number of holidays (i.e., holes in the mosaic).

The iterative spectral analyses employed to generate training signatures for classification showed that stands of wetland plants, especially different reeds, rushes, and vertical habit grasses, were spectrally inseparable. This observation is not unexpected. Past research, where spectral signatures of vegetation were collected using ground-based spectroradiometers, under natural illumination, concluded that reflectance properties of unique vegetation types do not depend on species, but rather on the plant phenology and the types of background materials. Within the AISA imagery, a variety of factors influence the spectral characteristics of the selected ROIs, including above-ground biomass, plant distribution within the sensor field of view, crown and foliar structure, within and betweenplant shadowing, and the background soil properties. With such a wide range of interacting physical parameters, many ROI signatures were not separable within one standard deviation of their means. For example, deciduous tree crowns were spectrally similar to dense grass cover or dense broad-leafed crops, and dead or dying forests were spectrally confused with wetland grasses or reeds that support necrotic stems and leaves. This inseparability produced a vegetation classification with a relatively low overall accuracy.

The high spatial resolution acquired by the AISA sensor for this project would suggest the potential for classifying the wetland vegetation classes with a greater level of detail and accuracy. The marginal quality of the final map suggests that smaller pixels do not necessarily result in a better classification. The

highly variable spectral response of adjacent pixels within the homogenous 
marsh grass communities illustrates this dilemma found in all high spatial resolution digital imagery. Homogeneity with respect to species composition may not mean homogeneity with respect to biomass distribution and foliar density. For example, S. patens can exist as uniform mats composed of both live and dead stems, as well as widely spaced clumps. Both densities of $S$. patens would be classified as salt meadow hay from ground surveys. However, the spectral response of a pixel within the high-density S. patens is significantly different from a pixel within the low-density $S$. patens. The spectral mixture of the low-density $S$. patens is easily confused with low-density stands of the other marsh grass species.

The best solution to classify spectrally similar species is to collect large numbers of ground truth plots, stratified primarily by composition and density. Additional variables needed for stratification to ensure a representative sample may include background materials, namely soils and water quality (i.e., clarity). From a practical standpoint, a comprehensive vegetation inventory over a small study area, perhaps no greater than few hundred acres, could supply the stratified sample needed for a quality image classification. However, for an area as large as the Refuge, such a sample is relatively impractical to provide enough useful ground truth data for a useable thematic map from high-resolution imagery. A more practical approach may be to divide the Refuge into a set of smaller areas, or tiles, concentrating ground truth data collection within project specific management or study sites.

The implementation of high resolution airborne digital remotely sensed imagery, both multispectral and hyperspectral, is advancing with the enhancements to both the sensor systems and the computer hardware and software needed for data processing. The obstacles encountered in this applied research effort significantly affected the final product, in terms of both the accuracy of the thematic map and the time required to complete the project. As these technologies improve, it will be important for Corps personnel to understand the basic principles of how the imagery are collected, how the imagery are post-processed, and how the final map product is created. 


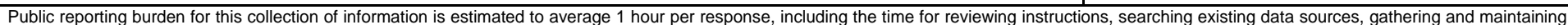

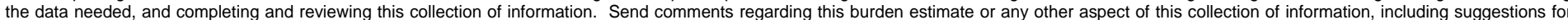

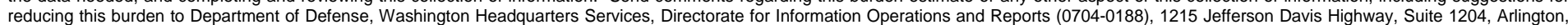

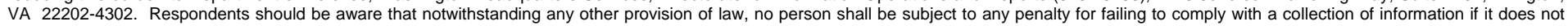
display a currently valid OMB control number. PLEASE DO NOT RETURN YOUR FORM TO THE ABOVE ADDRESS.
1. REPORT DATE $(D D-M M-Y Y Y Y)$ 2. REPORT TYPE
February 2005
Final
DATES COVERED (From - To)

4. TITLE AND SUBTITLE

USING HIGH SPATIAL RESOLUTION DIGITAL IMAGERY

5a. CONTRACT NUMBER

5b. GRANT NUMBER

5c. PROGRAM ELEMENT NUMBER

6. AUTHOR(S)

5d. PROJECT NUMBER

Michael V. Campbell, Robert L. Fischer, Timothy Pangburn, and Mark J Hardenberg

5e. TASK NUMBER

5f. WORK UNIT NUMBER

7. PERFORMING ORGANIZATION NAME(S) AND ADDRESS(ES)

8. PERFORMING ORGANIZATION REPORT NUMBER

U.S. Army Engineer Research and Development Center

72 Lyme Road

ERDC TR-05-1

Hanover, NH 03755

9. SPONSORING I MONITORING AGENCY NAME(S) AND ADDRESS(ES)

10. SPONSOR/MONITOR'S ACRONYM(S)

Headquarters, U.S. Army Corps of Engineers

Washington, DC 20314-1000

11. SPONSOR/MONITOR'S REPORT NUMBER(S)

\section{DISTRIBUTION / AVAILABILITY STATEMENT}

Approved for public release; distribution is unlimited

13. SUPPLEMENTARY NOTES

\section{ABSTRACT}

This document is the culmination of over 3 years worth of applied research. The overall objective of this multi-year investigation was to assess the utility of high spatial resolution digital imagery to Corps civil works operations. The sources of remotely sensed data used in this research effort include digital airborne multispectral imaging technology, digital airborne hyperspectral imaging technology, and digital satellite multispectral images. System selection was based on the anticipated benefits derived from the acquisition and processing of the unique high spatial resolution images, from each remotely sensed data source, at each independent study site. The project developed and implemented four applications to evaluate the use of high resolution digital remotely sensed data in standard Corps operations. This document summarizes the four projects. The intended audience for this document includes natural resource managers, biologists, ecologists, GIS specialists, and others that are already using or are potentially planning to use high spatial resolution digital remotely sensed imagery for current or future projects. The image and map products, including both raster and vector formatted data, are not provided with this report. The authors can provide the reader access to all of the imagery and final maps.

\section{SUBJECT TERMS}

Digital image processing

High spatial resolution

16. SECURITY CLASSIFICATION OF:

\section{a. REPORT}

$\mathrm{U}$ b. ABSTRACT

U
Hyperspectral

Invasive species mapping Multispectral

Remote sensing

Wetlands mapping

\begin{tabular}{c|c|}
$\begin{array}{c}\text { 17. LIMITATION } \\
\text { OF ABSTRACT }\end{array}$ & $\begin{array}{c}\text { 18. NUMBER } \\
\text { OF PAGES }\end{array}$ \\
$\mathrm{U}$ & 105 \\
\hline
\end{tabular}

19a. NAME OF RESPONSIBLE PERSON

19b. TELEPHONE NUMBER (include area code) 